\title{
Ecological stochasticity and phage induction diversify bacterioplankton communities at the microscale
}

\author{
Rachel E. Szabo ${ }^{a, b}$, Sammy Pontrellic, Jacopo Grillid, Julia A. Schwartzman ${ }^{b}$, Shaul Pollak ${ }^{b}$, \\ Uwe Sauer ${ }^{c}$, Otto X. Corderob, \\ ${ }^{a}$ Microbiology Graduate Program, Massachusetts Institute of Technology, Cambridge, MA 02139, \\ USA. \\ ${ }^{\mathrm{b}}$ Department of Civil and Environmental Engineering, Massachusetts Institute of Technology, \\ Cambridge, MA 02139, USA. \\ 'Institute of Molecular Systems Biology, ETH Zürich, 8093 Zürich, Switzerland. \\ ${ }^{d}$ Quantitative Life Sciences, The Abdus Salam International Centre for Theoretical Physics, \\ 34151 Trieste, Italy.
}

*To whom correspondence may be addressed. Email: ottox@mit.edu

Author Contributions: RE Szabo designed and performed experiments; analyzed and interpreted data; and wrote the manuscript. S Pontrelli performed metabolomics and the related analysis. J Grilli developed the population dynamics model and interpreted data. JA Schwartzman designed and performed the multi-particle incubation experiment and analyzed particle images. $S$ Pollak developed the analysis pipeline for classifying MAGs into ecological roles. OX Cordero designed experiments, interpreted data, and wrote the manuscript. All authors edited and reviewed the manuscript.

Competing Interest Statement: The authors declare no competing interest.

\section{This PDF file includes:}

Main Text

Figures 1 to 5

Main References

Supplementary Text

Figures S1 to S19

Legends for Tables S1 to S7

Supplementary References

Separate supplementary materials for this manuscript include:

Tables S1 to S7 


\section{Abstract}

3 In many natural environments, microorganisms self-assemble around heterogeneously distributed

4 resource patches. The growth and collapse of populations on resource patches can unfold within

5 spatial ranges of a few hundred micrometers or less, making such microscale ecosystems hotspots

6 of biological interactions and nutrient fluxes. Despite the potential importance of patch-level

7 dynamics for the large-scale evolution and function of microbial communities, we have not yet been

8 able to delineate the ecological processes that control natural populations at the microscale. Here,

9 we addressed this challenge in the context of microbially-mediated degradation of particulate

10 organic matter by characterizing the natural marine communities that assembled on over one

11 thousand individual microscale chitin particles. Through shotgun metagenomics, we found

12 significant variation in microscale community composition despite the similarity in initial species

13 pools across replicates. Strikingly, a subset of particles was highly populated by rare chitin-

14 degrading strains; we hypothesized that their conditional success reflected the impact of stochastic

15 colonization and growth on community assembly. In contrast to the conserved functional structures

16 that emerge in ecosystems at larger scales, this taxonomic variability translated to a wide range of

17 predicted chitinolytic abilities and growth returns at the level of individual particles. We found that

18 predation by temperate bacteriophages, especially of degrader strains, was a significant contributor

19 to the variability in the bacterial compositions and yields observed across communities. Our study

20 suggests that initial stochasticity in assembly states at the microscale, amplified through biotic

21 interactions, may have significant consequences for the diversity and functionality of microbial

22 communities at larger scales.

\section{Significance Statement}

27 The biogeochemical consequences of the degradation of particulate organic matter by 28 microorganisms represent the cumulative effect of microbial activity on individual microscale 
resource patches. The ecological processes controlling community dynamics in these highly localized microenvironments remain poorly understood. Here, we find that complex marine communities growing on microscale resource particles diverge both taxonomically and functionally despite assembling under identical abiotic conditions from a common species pool. We show that this variability stems from bacteriophage predation and history-dependent factors in community assembly, which create stochastic dynamics that are spatially structured at the microscale. This microscale stochasticity may have significant consequences for the coexistence, evolution, and function of diverse bacterial and viral populations in the global ocean.

Introduction

A central challenge in microbial ecology is to connect the microscale world experienced by microbial cells to observations of large-scale community functions $(1,2)$. In many environments homogeneous, well-mixed cultures, but rather in diverse, spatially-structured assemblages, attached to surfaces and other cells in nutrient-dense patches on the order of $100 \mu \mathrm{m}$ in size. Patches often exist in otherwise nutrient-limiting environments, creating hotspots of ecological interactions and nutrient fluxes $(7,8)$.

A well-known example of micron-scale ecological hotspots is marine particulate organic 51 matter (POM), which is degraded by complex communities of bacteria, archaea, viruses, and

52 eukaryotes (9) with global biogeochemical consequences (10) (Fig. 1a). These interacting 53 community members can be broadly classified as primary degraders (that produce extracellular 54 enzymes to hydrolyze particle biopolymers), exploiters and scavengers (that are facilitated by 55 primary degraders) (11-14), and predators (such as bacteriophages (15) and grazers (16)). 56 Although these assemblages are often ephemeral, with organisms migrating through seawater 
57 from patch to patch, cells can undergo multiple generations of growth while residing on a single

58 patch of nutrient-rich POM. This implies that the evolution and ecological functions of POM-

59 associated microbes are heavily influenced by their dynamics and interactions on microscale

60 particles. However, little is known about the processes governing community assembly at these

61 scales.

62 A major obstacle to understanding the factors that control populations at the patch level is

63 the difficulty of characterizing natural microscale communities with high replication. Microbial

64 communities are usually sampled at spatial scales orders of magnitude larger than those relevant

65 for microbial life (2), which homogenizes their inherent patchiness and results in inconsistent

66 inferences about ecological interactions $(1,17)$. Recent technological advances now permit the

67 sequencing of only thousands of cells $(18,19)$, presenting an opportunity to systematically

68 characterize microbial populations in units more closely approximating in scale the ecological

69 contexts experienced by microbes.

70 Here, we leveraged high-replicate sequencing of individual microscale communities to

71 evaluate the outcomes of assembly processes without the confounding effects of standard

72 sampling procedures. We employed a hybrid natural-laboratory approach that paired the

73 complexity of environmental microbial species pools with the controllability of synthetic

74 microparticles as discrete resource patches $(11,12)$. We immersed 1222 individual hydrogel

75 particles $(85.0 \pm 24.0 \mu \mathrm{m}$ in diameter) made of chitin - a highly abundant biopolymer in marine POM

76 (9) - in samples of seawater containing microbes in their native states, which were then enriched

77 on particle surfaces. By incubating single particles separately under identical abiotic conditions,

78 each one became a microenvironment harboring a replicate community assembled from initially

79 similar species pools. We performed a comparative analysis across these microscale ecosystems

80 to investigate the natural variability in community composition and function among particles and to

81 identify biological processes that contribute to particle-level variability. 


\section{Results}

Bacterial community composition varies significantly across individual particles.

87 To quantify the variation in community states across replicate microscale ecosystems, we separately incubated single chitin particles in coastal seawater sampled from a common reservoir (Fig. 1b; Methods). Assembly outcomes were assessed by removing particles from the seawater at 13 time points over the course of 167 hours, a duration that aligns with previous measurements $(12,20)$ of particle lifetimes (Methods). Shotgun metagenomic sequencing of individual particleattached communities was used to construct metagenome-assembled genomes (MAGs), which were annotated to infer strains' potential ecological roles in a chitin-degrading community as primary degraders, chitooligosaccharide exploiters, or metabolic byproduct scavengers (Fig. 1b; Table S1; Methods). These MAGs served as the references for characterizing the community composition of each particle.

We found a remarkable degree of compositional variability across individual particle communities at the end of the time course ( $n=149$, after 154-167 hours of incubation). The distributions of taxon (MAG) relative abundances across these late-stage particles spanned more than three orders of magnitude (Fig. 2; Fig. S1a) and were approximately lognormal with a skew towards high frequencies (Fig. S2). As a result, the community states observed at the single-particle level diverged so significantly that the relative success of taxa across particles was poorly explained by their average abundances (Fig. S1b; SI Methods). To assess whether non-ecological factors, such as sampling bias in initial species pools, could have contributed to this compositional divergence, we compared the variability in communities across late-stage particles to that across unincubated aliquots of the seawater used as the inoculum (SI Methods). Inter-sample variation was significantly higher across particles than across seawater samples (Mann-Whitney $U$ test on Aitchison distances: $p=1.3 \times 10^{-13}$; Fig. S3), indicating that the observed variability stemmed more from the community assembly process than from differences across inocula. Because other 
110 technical sources of noise (Methods) also did not significantly impact the measured particle

111 compositions (Fig. S4) and all particles were chemically identical, we concluded that the variation

112 in taxon relative abundances across particles was due to biological and ecological factors that

113 amplified stochasticity in the initial assembly states of these communities.

114 The skew towards high frequencies in the relative abundance distributions implied that taxa

115 that were rare on average became dominant on a small number of particles (Fig. 2). As a result,

116 those particles harbored low-complexity communities (Fig. S5a-b) that diverged highly from the

117 average particle taxonomic composition (Fig. S5c). We termed the species that displayed this

118 phenomenon "jackpot taxa" for their simultaneous local success and global rarity (Methods). The

119 strains in this phylogenetically broad group of organisms included members of the

120 Enterobacterales, Cytophagales, Pseudomonadales, Flavobacteriales, Rhodobacterales,

121 Fibrobacterales, and Chitinophagales orders (Fig. S1, Table S1) and were mostly (87.9\%)

122 classified as chitin degraders. Jackpot taxa were more prevalent across late-stage particles than

123 other taxa that were equally rare across inocula (Mann-Whitney $U$ test: $p=7.1 \times 10^{-3}$; Fig. S5d),

124 indicating that the probability of their success on particles, while influenced by their scarcity in

125 seawater, was also determined by ecological factors during community assembly. Notably, while

126 taxon-specific interactions did not explain the abundance patterns observed across particles (Fig.

127 S6; SI Methods), the most variable strains were likely to be degraders enriched in genes encoding

128 chitinases (Fig. 2; Fig. S7; coefficient of variation vs. chitinase copy number, Spearman's $\rho=0.44$,

$\left.129 p=8.5 \times 10^{-7}\right)$. These observations indicated that the conditional success of specialized degraders

130 from a diverse initial species pool contributed to the differentiation of the many rare community

131 states found at the single-particle level.

133 Taxonomic variability translates to divergent community-level productivity.

134 In contrast to the functionally similar gene content profiles predicted when microbial ecosystems 135 are characterized at the macroscale $(21,22)$, we found that the communities formed on particles 136 in individual microscale ecosystems were highly functionally divergent (Fig. 3a; Fig. S8). By the 137 end of the time course, most particles (63.8\%) - and especially particles dominated by jackpot taxa 
138 - harbored majority-degrader communities (Fig. 3a), highlighting the importance of degraders for 139 establishing and maintaining chitin-associated communities. However, the percentage of putative 140 degraders on each late-stage particle was as low as $13.1 \%$ and as high as $97.3 \%$, indicating that 141 chitin degrading communities did not self-assemble to "optimized" or conserved ratios of ecological 142 roles after a fixed incubation period. Read mapping to chitinase protein sequences rather than 143 MAGs supported our interpretation that variability in the estimated proportion of degraders was not 144 due to the use of MAGs as reference genomes (Fig. S9; SI Methods). We hypothesized that this 145 extensive variability in community composition, primed by stochasticity in assembly processes, 146 could have had significant consequences for overall community function.

147 Consistent with this hypothesis, individual particles sustained highly variable particle148 attached biomass levels that were correlated with their community compositions (Fig. S10). The 149 number of bacterial cells in each late-stage community, estimated using qPCR of the 16S rRNA 150 region (Methods), ranged from approximately 1,000 to nearly 200,000 cells (Fig. $3 \mathrm{~b}$ ) and was 151 strongly correlated with the overall frequency of degraders (Spearman's $\rho=0.45, p=1.6 \times 10^{-8}$ ). 152 Accordingly, particles that displayed the jackpot phenomenon had significantly higher cell counts 153 (Fig. 3b; Mann-Whitney $U$ test: $p=2.3 \times 10^{-7}$ ), revealing that jackpot taxa were dominant not only in 154 terms of relative abundances but also absolute abundances. The distribution of cells per particle 155 was approximately lognormal with a skew towards low cell numbers, indicating that some particles 156 were highly productive while others harbored small populations even by the end of the incubation, 157 as corroborated by visualizing particle-attached cells using a DNA stain (Fig. 3c; Methods). 158 Importantly, the initial colonization of single particles incubated together in the same volume of 159 seawater, rather than individually, resulted in particle-associated cell biomass that also spanned 160 several orders of magnitude (Fig. S11; SI Methods). This variability in initial particle colonization 161 was observed across a range of particle densities 15-140 times more concentrated than the 162 conditions of the individual particle incubations, indicating that phenomena such as jackpot 163 colonization are not specific to the environmental regime established in our separate microscale 164 ecosystems. Collectively, these results suggested that a strain's growth was highly influenced by 
165

166

167

168

169

170

171

172

173

174

its assembly context, raising the question of which biological or ecological factors could explain the large variance in species compositions and consequent yields across replicate particles.

\section{Predation by bacteriophages contributes to variability in community composition and yield.}

Our observation that most $(63.7 \%)$ of our MAGs contained sequences homologous to those of bacteriophages led us to investigate whether these entities impacted the abundances of bacteria on single particles. Bacteriophages (or phages, i.e. viruses that infect bacteria) are ubiquitous and abundant in marine ecosystems, making predation by phages one of the primary forms of top-down control of bacterial populations (23). High viral densities have been measured on marine particles relative to ambient seawater (15), but it is unknown to what extent this represents passive adsorption as opposed to active proliferation with impacts on bacterial growth in a natural, particleassociated context. Therefore, we sought to identify populations of actively replicating phages within the single particle communities to determine if heterogeneous phage predation could explain the variability in community composition and yield.

To detect replicating phages, we first classified contigs in our metagenomic dataset as phage-derived or bacteria-derived using tools $(24,25)$ that annotate phages from mixed metagenomes (Methods). We reasoned that contigs classified as phage-derived, especially those belonging to the genomes of temperate phages, were likely to be binned into the MAGs of their bacterial hosts. Phage $k$-mer signatures tend to be more similar to those of their specific hosts than to those of random bacteria $(25,26)$, and phages in a lysogenic cycle will have the same sequencing read coverage patterns as their hosts across samples. Therefore, phages that were lysogenic in most single-particle communities would tend to be binned with their hosts and have similar coverage levels, reflected in an inferred virus-to-microbial cell ratio (VMR) close to 1 (Fig. 4a, top left). In contrast, phages in a productive cycle (lytic or chronic) would have higher coverage than their hosts because of the multiple virion copies produced per bacterial cell $(27,28)(\mathrm{Fig} .4 \mathrm{a}$, top right). Therefore, we considered a phage-derived contig to be productive in a sample if it was one of the most highly covered elements of its MAG (Methods). 
Through this pipeline, we identified 256 phage contigs with coverage patterns consistent with lysogenic infections in all samples and 263 phage contigs with coverage patterns consistent with productive infections in a subset of samples (Table S2). Because our approach relied on comparisons between co-binned phages and MAGs, phages that exclusively employ a lytic cycle were unlikely to be detected. The VMRs of three representative examples of lysogenic and productive phage contigs are shown for each particle in Figure 4b. Comparing the coverage patterns of phage- and bacteria-derived contigs provided evidence that variable phage coverage was not due to sequencing noise, lending confidence to our estimates of VMRs for specific phages (Fig. S12; SI Methods). Using the VMRs of individual productive phages, we calculated the total productive VMR per particle as a measure of overall phage replication in each community (Methods).

The total productive VMRs of particle-associated communities sharply increased during the early stages of particle incubation in seawater (Fig. 4c), consistent with the phenomenon of rapid bacterial growth and high host densities driving the lysogeny-lysis switch in some phages (29-33). The mean productive VMR was lowest for the initial seawater inocula and rose sharply until the middle of the incubation period (59 hours), suggesting that phages became induced as their particle-associated hosts began to grow. Concomitant with this increase in productive VMRs, 210 hours of incubation, followed by a decrease in metabolite concentrations (Fig. 4d; Fig. S13; Table 211 S3; Methods). These observations could be explained by metabolite release upon the initiation of 212 bacterial growth (34) or lysis by phages (35) and by subsequent metabolite consumption by the 213 remaining viable bacteria (36). The coinciding timescales of metabolite liberation and rising VMRs 214 are consistent with our hypothesis that a particle-associated lifestyle among bacteria promoted 215 phage proliferation; therefore, we sought to assess the impact of variable phage induction on each 216 community's composition and consequent yield. 
populations contributed the most to this signal, indicating that strains among this trophic level may have been especially prone to phage activation (Fig. 4e inset; Fig. S14). Importantly, jackpot degrader taxa had lower productive VMRs than non-jackpot degraders (Fig. S15a; Mann-Whitney

$223 U$ test: $\left.p=1.3 \times 10^{-49}\right)$. This translated to jackpot particles having significantly lower productive VMRs 224 than non-jackpot particles (Mann-Whitney $U$ test: $p=4.3 \times 10^{-8}$ ), even controlling for differences in cell counts between these groups of communities (ANCOVA: $F(1,139)=16.92, p=4.1 \times 10^{-4}$, partial $\eta^{2}=0.09$; Fig. S15b). Therefore, jackpot degraders may have been locally successful on a minority of particles in part because they experienced less predation, supporting the hypothesis that topdown population control by phages contributed to the large variability in the bacterial compositions and thus yields observed across communities.

While cell counts were significantly correlated with both phage abundances and community compositions, these features explained, respectively, 23\% (Fig. 4e) and 34\% (Fig. S10) of the observed variation in yields, indicating that other factors also contributed to variable growth returns. Therefore, we sought a more general framework in which to understand the key quantitative features of the data - namely, the lognormal-like distributions of relative taxon abundances (with right skews consistent with jackpot taxa) and of absolute cell abundances (with a left skew corresponding to low-biomass communities). Incorporating (i) stochastic cell arrival on particles, (ii) degraders as population founders, and (iii) noisy growth rates into a simple mathematical model of community development on single particles was sufficient to reproduce these features (Figs. S16S19; SI Text). Taken together with our experimental data, this model indicates that the biological

240 processes which contribute to the stochasticity of particle colonization and growth rates - and 241 especially those processes that affect degraders - will result in variable growth returns for strains 242 across particles. 
While there is an abundance of evidence showing that the marine environment as experienced by microbial cells is biologically, chemically, and physically heterogeneous (7),

249 characterizing the ecological processes controlling community assembly and development at these 250 scales remains a fundamental challenge, particularly in situ. Our study takes a step toward 251 addressing this problem using a hybrid natural-laboratory experiment that monitored the assembly 252 outcomes of complex marine communities across hundreds of individual chitin-based resource 253 particles. In accordance with prior work demonstrating small-scale heterogeneity on aquatic 254 resource particles (37), we found that bacterial compositions and absolute abundances varied to 255 such an extent across replicate particles that key community features - namely, species 256 composition and functional potential - were not conserved. Our results contrast with those of 257 previous studies $(11,13)$ that describe rapid ecological successions within particle systems that 258 are reproducible across batches. Despite this apparent reproducibility, biomass distributions in our 259 single-particle and our multi-particle incubations suggest that particle colonization is likely 260 heterogeneous in both systems. Thus, the reproducible dynamics previously observed in particle 261 systems could reflect the increasingly homogenizing effect of exchange between particles over 262 time or the mean of a process that is highly variable on the individual-particle scale. Future work is 263 required to determine the effect of dispersal and "cross-colonization" on the dynamics of particle 264 systems.

265 Stochastic factors are anticipated to strongly influence community assembly for 266 populations that are localized to small scales (38), such as in the microscale ecosystems on 267 resource particles $(12,20)$. The first step in community assembly - the arrival of cells to a particle $268-$ is an intrinsically random process dependent on encounter probabilities. Our population dynamics 269 model demonstrated that historical contingencies (created by stochastic arrival times and the 270 growth dependency of non-degraders on degraders) magnified through noisy growth rates were 271 sufficient to reproduce the distributions of bacterial abundances observed across individual 272 particles. Because this chitin microparticle ecosystem is subject to conditions that have been shown 273 to promote strong priority effects (e.g. a large regional species pool, rapid local growth dynamics, 274 high resource overlap, and a dependence of late-arriving organisms on early-arriving ones) (38), 
275 we hypothesize that biotic factors amplified this initial stochasticity in each assembly context and

276 influenced subsequent community development.

277 One key biological contributor to noisy growth returns may have been variable predation

278 by temperate bacteriophages. Phages became increasingly and differentially activated during

279 community development on particles, with elevated virus-to-microbial cell ratios (VMRs) in low-

280 biomass communities implicating phage-mediated lysis as one factor explaining the biomass

281 variability on late-stage particles. These results align with those of previous studies documenting

282 extensive variation in VMRs at small spatial scales (23) and an inverse relationship between VMRs

283 and cell densities in marine environments (39). Because phage induction was significantly less

284 associated with jackpot degrader strains, we hypothesize that the jackpot phenomenon -

285 characterized by globally rare yet locally productive degraders - was partially a reflection of lower

286 levels of phage-driven population collapse in those community contexts. Therefore, top-down

287 control by phages may link the highly variable community compositions and yields observed among

288 particles.

A synthesis of our mathematical model with our observations of bacterial and phage abundances suggests a conceptual framework for key processes promoting variability in microscale community composition and function (Fig. 5). We posit that stochastic arrival on particles diversifies initial assembly states; that the timescale and magnitude of degrader colonization determine the extent to which scavengers and exploiters are supported; and that phage induction and subsequent host lysis, primarily among degraders, contribute to noisy growth returns. Therefore, in this conceptual framework, the high-biomass jackpot particles are those in which degraders arrive early and resist phage induction, leading to high relative and absolute degrader abundances (Fig. 5, top). By contrast, low-biomass particles are those in which degraders are not able to proliferate, either because phage induction leads to their population collapse (Fig. 5, middle) or because they become established on a particle relatively late (Fig. 5, bottom). In addition to the growing body of evidence that marine aggregates can stimulate the 
303 that conditionally employ both lytic and lysogenic cycles). In marine environments, lysogeny is

304 promoted under conditions that limit bacterial growth while the lytic cycle is favored during periods

305 of high bacterial activity $(29,41,42)$, indicating that rapid host growth and abundance can regulate

306 the lysogeny-lysis switch in some temperate phages (30-33). Therefore, in a patchy nutrient

307 landscape, temperate phages may employ lysogeny as a survival strategy when their bacterial

308 hosts are at low densities and are foraging for nutrients, hitchhiking with their hosts onto resource

309 particles. Robust bacterial growth on particles may induce prophages at a time when abundant

310 host resources can be co-opted and many susceptible cells are nearby, resulting in lytic

311 suppression of the bacterial population and the release of virions into the surrounding seawater.

312 Factors such as the variable presence of prophages in the flexible genomes of strains growing on

313 different particles (43), the co-occurrence of bacterial competitors that trigger induction $(44,45)$,

314 and phenotypic heterogeneity resulting in differential induction $(46,47)$ may all contribute to the

315 varying levels of phage activation observed on individual particles in our microscale ecosystems.

316 Further research is required on the mechanisms underlying prophage induction in complex

317 communities in order to understand how lysogeny and lysis on particle hotspots shape the

318 dynamics of marine microbial communities.

319 Our observations of wild marine communities, though made in a laboratory setting, may

320 provide insights on the ecosystem-level consequences of microscale stochastic assembly

321 dynamics. First, the stochasticity in bacterial growth, amplified through spatial structuring at the

322 microscale, may promote the maintenance of a diverse regional species pool. This is because the

323 variability in growth returns can effectively offset differences in relative fitness between competing

324 strains or species (48). Second, the variability in microscale community states could be reflected

325 in larger-scale biogeochemical patterns since the cumulative process of POM degradation can be

326 approximated as the sum of degradation events on individual particles. We found that late-stage

327 communities did not converge to a fixed proportion of chitin degraders or to a fixed amount of

328 biomass per particle; both measures are positively correlated with the rate of particle degradation

329 (12), suggesting that historical contingencies in community assembly promote functional 330 divergence $(38,49)$. These results contrast with those of previous studies on the replicability of 
331 microbial community assembly at the functional level $(21,22)$ likely because of the homogenizing

332 effect of macroscale sampling. Intriguingly, the lognormal-like distribution of biomass on individual

333 particles aligns with observations and predictions of lognormally-distributed global marine organic

334 matter export and remineralization rates; these distributions may repeatedly emerge as a reflection

335 of the multiplicative effects of stochastic variables in ecological settings (50-52). Although our

336 experimental system significantly simplified the process of POM degradation in the ocean, our

337 approach provides a quantitative link between the microscale and larger-scale processes,

338 highlighting the importance of considering local variability when investigating the mechanisms

339 behind microbial community development in a spatially structured environment.

Materials and Methods

Abridged Methods are provided below; details and additional information are provided in SI Methods.

Seawater collection and individual chitin particle incubation. Nearshore coastal seawater was collected from Nahant, MA; filtered $(63 \mu \mathrm{m})$ to remove large particulate matter; gently concentrated via centrifugation at $4000 \times g$ for 5 minutes; and aliquoted for incubations and sequencing. Chitin magnetic particles (New England Biolabs, \#E8036L) were washed in sterile artificial seawater (Sigma-Aldrich, \#S9883) and individually selected beneath a dissecting microscope in a laminar flow hood. Single chitin particles $(85.0 \pm 24.0 \mu \mathrm{m}$ in diameter) were transferred to sterile 96-well 353 plates (Thermo Fisher, \#AB0600L), with one chitin particle per well. Plates were inoculated 354 consecutively with $175 \mu \mathrm{L}$ of filtered, centrifuged seawater per well; sealed (VWR, \#89092-056); 355 and rotated end-over-end $(7.5 \mathrm{rpm})$ at room temperature. The particles in an entire plate were 356 harvested at each time point (after 12, 22.75, 34.5, 46, 59, 69, 82, 92, 103, 116.75, 113, 153.5, and 357166.5 hours of incubation) by inspection and pipetting under a dissecting microscope in a laminar 
containing TE buffer and stored at $-20^{\circ} \mathrm{C}$. The seawater surrounding each harvested particle was also saved in $96-$ well plates and stored at $-20^{\circ} \mathrm{C}$.

Mock communities and negative controls. To quantify the technical error associated with creating metagenomic libraries from low DNA inputs, mock communities were simulated by combining the DNA of two strains previously isolated from a chitin particle enrichment (11). Libraries from three technical replicates of mock communities totaling 50pg or 5pg of DNA (SI Methods), as well as from six negative controls (containing only nuclease-free water), were prepared and analyzed with the same protocols used for individual chitin particle-attached communities.

DNA extraction and metagenomic sequencing. DNA extractions were performed for twelve

$371175 \mu \mathrm{L}-$ volume aliquots of the initial, unincubated seawater and for particles harvested after 34.5,

$37259,103,116.75,113,153.5$, and 166.5 hours of incubation (see Table S5 for sample metadata).

373 DNA was extracted from all samples with the Agencourt DNAdvance Genomic DNA Isolation Kit

374 (Beckman Coulter; modifications noted in SI Methods). Metagenomic libraries were prepared with

375 the Nextera XT DNA Library Prep Kit and index primers (Illumina) using the protocol developed by

376 Rinke et al. (18) for low DNA inputs (SI Methods). Libraries were quantified on an Agilent 4200

377 TapeStation system with High Sensitivity D5000 ScreenTapes (Agilent Technologies) and pooled

378 by time point in equimolar amounts. Sequencing was performed on an Illumina HiSeq 2500

379 machine (250bp paired-end reads) at the Whitehead Institute for Biomedical Research (Cambridge, $380 \mathrm{MA})$.

Metagenome-assembled genome (MAG) generation, taxonomic assignment, and role

383 classification. Raw sequencing reads were quality trimmed with Trimmomatic v0.36 (53). Reads

384 mapping to the PhiX and human genomes were filtered out using BBDuk v38.16 (54) and BBMap

385 v38.16, respectively (SI Methods). Trimmed, filtered reads that were error-corrected using 386 BayesHammer (55) were pooled within each time point and co-assembled using MEGAHIT v1.2.9 
387 (56). Bins were generated with MaxBin v2.2.7 (57) and CONCOCT v1.1.0 (58); consolidated and

388 filtered using DAS Tool v1.1.1 (59); and evaluated for completeness and contamination using

389 CheckM v1.1.2 (60). The resulting 251 bins were used as reference MAGs ( $\geq 50 \%$ complete, $\leq 10 \%$

390 contaminated; median completeness 93.7\%, median contamination 3.9\%; Table S1). Highly similar

391 MAGs obtained from separate co-assemblies were grouped into 132 clusters (SI Methods). MAG

392 taxonomic classifications were made using GTDB-Tk v1.1.1 (61). MAGs were functionally

393 annotated using a custom database of profile hidden Markov models (HMMs) of proteins involved

394 in growth on chitin (SI Methods; Table S6). Ecological roles for MAGs (as degraders,

395 chitooligosaccharide exploiters, or metabolic byproduct scavengers) were defined based on the

396 gene content patterns observed for sequenced and phenotyped (14) strains previously isolated

$397(11,13)$ from particle enrichments (SI Methods).

398

399 Read mapping to MAGs for relative abundance estimation. Trimmed, filtered reads were

400 mapped competitively against the MAGs generated from sequencing particle-attached

401 communities, initial seawater samples, and negative controls. Read mapping was performed using

402 the approach described in Leventhal et al. (62) (SI Methods). Reads that best mapped to predicted

403 contaminant MAGs (SI Methods) were removed from consideration. MAG relative abundances

404 were calculated for each sample by (1) tallying the hits to all MAGs in each MAG cluster; (2)

405 normalizing the tally by the average genome length of all MAGs in each MAG cluster; and (3)

406 dividing the normalized tallies for each MAG cluster by their sum for each sample. Therefore, for

407 MAGs clustered together based on similarity, their relative abundances are represented in that of

408 the entire MAG cluster to which they belong; this calculation circumvents the artificial

409 underestimation of MAG relative abundances that would otherwise be obtained with a non-

410 dereplicated reference set. The relative abundances of organisms occupying the three ecological

411 roles (degrader, exploiter, scavenger) on each particle were calculated by summing the relative

412 abundances of MAGs classified into each role.

413 
414 Definitions of jackpot MAGs and jackpot particles. A jackpot score was calculated for each

415 MAG cluster to quantitatively reflect the properties of rarity across most particles and dominance

416 on a few particles (SI Methods) such that MAGs with high scores strongly displayed the jackpot

417 phenomenon. Each particle's jackpot score was calculated as the weighted average of MAG

418 jackpot scores (i.e. the sum of the relative abundance of each MAG cluster multiplied by its jackpot

419 score). Particles with high jackpot scores and low Pielou's evenness were categorized as “jackpot

420 particles" (SI Methods).

422 Bacteriophage analyses. Binned contigs were classified as phage-derived or bacteria-derived 423 using VirSorter v1.0.3 with its RefSeqABVir database (24) and VirFinder v1.1 (25), two tools 424 designed to detect phage sequences among mixed metagenomes (SI Methods). We used a read coverage-based approach to categorize phage-derived contigs as productive or lysogenic in 426 particle-attached communities (Table S2; see SI Methods for analysis controls). Based on read 427 mapping to MAGs, per-base coverage values for all binned contigs were computed with BEDTools 428 v2.27.0 (63) and were used to calculate contig-wide average coverage values. For each MAG and 429 for each sample, a phage-derived contig was considered to be productive if its coverage was 430 greater than the coverage of the $95^{\text {th }}$ percentile bacteria-derived contig in the same MAG; 431 otherwise, it was considered to be lysogenic in that sample. The VMR of an individual phage contig 432 in one sample is defined as the phage contig coverage divided by average coverage of the MAG 433 with which it is binned (which was calculated using only the bacteria-derived contigs). Total VMRs $434-$ i.e. the total number of phage copies relative to the total number of bacterial MAG copies in an 435 entire sample - were calculated separately for productive and lysogenic phage contigs. The total 436 productive VMR for a sample was defined as: 
441 where $n$ is the number of MAGs found in a sample. This calculation is equivalent to

where $n$ is the number of MAGs found in a sample. Total lysogenic VMRs were calculated using 446 the same formula while considering only lysogenic-annotated contigs. VMRs for each ecological 447 role (i.e. for the subpopulation in a community that belongs to one of the three roles of degrader, 448 exploiter, or scavenger) were calculated using the same formula considering only the MAGs of 449 each role and their associated phages.

Cell count estimation. Bacterial DNA extracted from individual particle-attached communities was quantified through qPCR of the 16S rRNA gene using the Femto Bacterial DNA Quantification Kit

453 (Zymo Research), which has a lower limit of detection of 20fg. Two sets of standards and negative 454 controls were included in each qPCR run. The number of bacterial cells for each particle was estimated from the absolute DNA amounts based on measurements indicating a mean of $2.5 \mathrm{fg}$

456 DNA per bacterial cell in seawater samples (64).

458 Imaging of chitin particle colonization. Subsets of chitin particles incubated individually in 459 seawater were stained at time points by adding the DNA stain SYTO9 (Invitrogen, \#S34854) at a 460 final concentration of $500 \mathrm{nM}$ directly to the particle incubations. Particles were incubated in the 461 dark at room temperature for 15 minutes before being mounted separately on microscope slides 462 and imaged with a Zeiss epifluorescence microscope at 100X magnification.

464 Metabolomics. We performed untargeted metabolomics of the seawater that surrounded each 465 harvested chitin particle and of the initial, unincubated seawater (SI Methods). We used a binary 466 LC pump (Agilent Technologies) and an MPS2 Autosampler (Gerstel) coupled to an Agilent 6520 
time-of-flight mass spectrometer (Agilent Technologies) operated in negative mode, at $2 \mathrm{GHz}$, extended dynamic range, with an $\mathrm{m} / \mathrm{z}$ (mass/charge) range of $50-1000$. Ions (Table S3) were

469 annotated against a curated library of metabolites present in marine microbes, based on the BioCyc 470 database (65). For metabolites that exceeded the limit of detection (SI Methods), the intensities of 471 each ion were normalized between 0 (the limit of detection) and 1 (the highest measured intensity 472 of a given ion). Weighted ion intensities for each timepoint were calculated by taking the sum of all 473 normalized intensities of ions in all samples for each timepoint.

Data Sharing Plans

478 All data will be made publicly available before publication. Sequencing data will be deposited to the 479 National Center for Biotechnology Information (NCBI) as a BioProject, with raw reads uploaded to 480 the Sequence Read Archive (SRA) and metagenome-assembled genomes (MAGs) uploaded to 481 the Whole Genome Shotgun (WGS) database. All mass spectra files from the metabolomics will 482 be accessible from MassIVE (ftp://MSV000087936@massive.ucsd.edu) before publication. MAG 483 relative abundances for each sample and metadata for samples, MAGs, phages, and detected 484 metabolites are provided as Supplementary Tables. All code and files used to generate figures will 485 be made available at personal GitHub pages before publication.

\section{Acknowledgments}

490 We extend our gratitude to all past and present members of the Cordero lab for their support and 491 critical feedback, as well as members of the Simons Collaboration on Principles of Microbial 492 Ecosystems for stimulating discussions. In particular, we would like to thank: Manoshi S. Datta, for 493 contributing to the genesis of this project; José T. Saavedra, for developing the DNA extraction and 494 metagenomic library preparation pipeline for single particle-attached communities; Gabriel E. 
495 Leventhal and Jakob Russel for bioinformatic mentorship and assistance; Matti Gralka, for 496 feedback on analyses and this manuscript; Elise Ledieu, for quantifying chitin particle sizes; 497 Anthony Gaca, for advice on metagenomic library preparation; Fatima Aysha Hussain, for feedback 498 on the bacteriophage analysis; Emily Zakem, for insights on global particle remineralization rates; 499 Akshit Goyal, for comments on this manuscript; and Sara Szabo and William Mandella, for 500 assistance with seawater sampling. This material is based upon work supported by the National 501 Science Foundation Graduate Research Fellowship under Grant No. \#174530. This project was 502 supported by the Simons Collaboration: Principles of Microbial Ecosystems (PriME) award number 503 542395. S Pontrelli was supported by a grant from the Simons Foundation (ID608247) as part of 504 PriME. S Pollak was supported by the EMBO ALTF Grant No. \#800-2017.

\section{Main References}

508

1. O. X. Cordero, M. S. Datta, Microbial interactions and community assembly at microscales. Curr Opin Microbiol 31, 227-234 (2016).

2. D. R. Nemergut, et al., Patterns and Processes of Microbial Community Assembly. Microbiol Mol Biol R 77, 342-356 (2013).

3. A. G. O'Donnell, I. M. Young, S. P. Rushton, M. D. Shirley, J. W. Crawford, Visualization, modelling and prediction in soil microbiology. Nat Rev Microbiol 5, 689-699 (2007). direct electron transfer in methanotrophic consortia. Nature 526, 531-535 (2015).

5. G. Gonzalez-Gil, C. Holliger, Aerobic granules: Microbial landscape and architecture, stages, and practical implications. Appl Environ Microb 80, 3433-3441 (2014).

6. J. L. M. Welch, B. J. Rossetti, C. W. Rieken, F. E. Dewhirst, G. G. Borisy, Biogeography of a human oral microbiome at the micron scale. Proc National Acad Sci 113, 791-800 (2016).

7. R. Stocker, Marine microbes see a sea of gradients. Science 338, 628-633 (2012). 
8. L. M. Dann, et al., Microbial micropatches within microbial hotspots. Plos One 13, 1-22 (2018).

9. H. Dang, C. R. Lovell, Microbial Surface Colonization and Biofilm Development in Marine Environments. Microbiol Mol Biol R 80, 91-138 (2016).

10. N. Jiao, et al., Microbial production of recalcitrant dissolved organic matter: Long-term carbon storage in the global ocean. Nat Rev Microbiol 8, 593-599 (2010).

11. M. S. Datta, E. Sliwerska, J. Gore, M. F. Polz, O. X. Cordero, Microbial interactions lead to rapid micro-scale successions on model marine particles. Nat Commun 7, 1-7 (2016).

12. T. N. Enke, G. E. Leventhal, M. Metzger, J. T. Saavedra, O. X. Cordero, Microscale ecology

13. T. N. Enke, et al., Modular Assembly of Polysaccharide-Degrading Marine Microbial Communities. Curr Biol 29, 1528-1535.e6 (2019).

14. S. Pontrelli, et al., Hierarchical control of microbial community assembly by specialists. bioRxiv [Preprint] (2021). https://doi.org/10.1101/2021.06.22.449372 (accessed 24 September 2021).

15. M. G. Weinbauer, et al., Viral ecology of organic and inorganic particles in aquatic systems: Avenues for further research. Aquat Microb Ecol 57, 321-341 (2009).

16. M. Simon, H. P. Grossart, B. Schweitzer, H. Ploug, Microbial ecology of organic aggregates in aquatic ecosystems. Aquat Microb Ecol 28, 175-211 (2002).

17. D. W. Armitage, S. E. Jones, How sample heterogeneity can obscure the signal of microbial interactions. Isme J 13, 2639-2646 (2019).

18. C. Rinke, et al., Validation of picogram- and femtogram-input DNA libraries for microscale metagenomics. Peerj 2016, 1-28 (2016).

19. R. U. Sheth, et al., Spatial metagenomic characterization of microbial biogeography in the gut. Nat Biotechnol 37, 877-883 (2019). 
20. M. H. Iversen, H. Ploug, Temperature effects on carbon-specific respiration rate and sinking velocity of diatom aggregates - potential implications for deep ocean export processes. Biogeosciences 10, 4073-4085 (2013). microbial communities. Nat Ecol Evol 1, 1-12 (2016).

22. C. Huttenhower, et al., Structure, function and diversity of the healthy human microbiome. Nature 486, 207-214 (2012).

23. M. Breitbart, C. Bonnain, K. Malki, N. A. Sawaya, Phage puppet masters of the marine microbial realm. Nat Microbiol 3, 754-766 (2018).

24. S. Roux, F. Enault, B. L. Hurwitz, M. B. Sullivan, VirSorter: Mining viral signal from microbial genomic data. Peerj 2015, 1-20 (2015).

25. J. Ren, N. A. Ahlgren, Y. Y. Lu, J. A. Fuhrman, F. Sun, VirFinder: a novel k-mer based tool for identifying viral sequences from assembled metagenomic data. Microbiome 5, 69 (2017).

26. N. A. Ahlgren, J. Ren, Y. Y. Lu, J. A. Fuhrman, F. Sun, Alignment-free d2* oligonucleotide frequency dissimilarity measure improves prediction of hosts from metagenomicallyderived viral sequences. Nucleic Acids Res 45, 39-53 (2017).

27. K. Kieft, K. Anantharaman, Deciphering active prophages from metagenomes. bioRxiv [Preprint] (2021). https://doi.org/10.1101/2021.01.29.428894 (accessed 24 September 2021).

28. R. F. von Boeselager, E. Pfeifer, J. Frunzke, Cytometry meets next-generation sequencing - RNA-Seq of sorted subpopulations reveals regional replication and iron-triggered prophage induction in Corynebacterium glutamicum. Sci Rep-uk 8, 1-13 (2018).

29. J. H. Paul, Prophages in marine bacteria: Dangerous molecular time bombs or the key to survival in the seas? Isme J 2, 579-589 (2008).

30. J. R. Brum, B. L. Hurwitz, O. Schofield, H. W. Ducklow, M. B. Sullivan, Seasonal time bombs: Dominant temperate viruses affect Southern Ocean microbial dynamics. Isme J 10, 437-449 (2016). 
577

578

579

31. M. Touchon, A. Bernheim, E. P. C. Rocha, Genetic and life-history traits associated with the distribution of prophages in bacteria. Isme J 10, 2744-2754 (2016).

32. J. E. Silpe, B. L. Bassler, A Host-Produced Quorum-Sensing Autoinducer Controls a Phage Lysis-Lysogeny Decision. Cell 176, 268-280.e13 (2019).

33. L. Laganenka, et al., Quorum sensing and metabolic state of the host control lysogenylysis switch of bacteriophage T1. Mbio 10, 3-8 (2019).

34. B. E. Noriega-Ortega, et al., Does the chemodiversity of bacterial exometabolomes sustain the chemodiversity of marine dissolved organic matter? Front Microbiol 10, 1-13 (2019).

35. N. Y. D. Ankrah, et al., Phage infection of an environmentally relevant marine bacterium alters host metabolism and lysate composition. Isme J 8, 1089-1100 (2014).

36. S. Blasche, et al., Metabolic cooperation and spatiotemporal niche partitioning in a kefir microbial community. Nat Microbiol 6, 196-208 (2021).

37. M. Bizic-lonescu, D. Ionescu, H.-P. Grossart, Organic particles: heterogeneous hubs for microbial interactions in aquatic ecosystems. Front Microbiol Accepted, 1-15 (2018).

38. T. Fukami, Historical Contingency in Community Assembly: Integrating Niches, Species Pools, and Priority Effects. Annu Rev Ecol Evol Syst 46, 1-23 (2015).

39. C. H. Wigington, et al., Re-examination of the relationship between marine virus and microbial cell abundances. Nat Microbiol 1, 4-11 (2016).

40. L. Riemann, H. P. Grossart, Elevated lytic phage production as a consequence of particle colonization by a marine Flavobacterium (Cellulophaga sp.). Microbial Ecol 56, 505-512 (2008).

41. M. G. Weinbauer, I. Brettar, M. G. Höfle, Lysogeny and virus-induced mortality of bacterioplankton in surface, deep, and anoxic marine waters. Limnol Oceanogr 48, 14571465 (2003).

42. J. P. Payet, C. A. Suttle, To kill or not to kill: The balance between lytic and lysogenic viral infection is driven by trophic status. Limnol Oceanogr 58, 465-474 (2013).

43. B. C. M. Ramisetty, P. A. Sudhakari, Bacterial "grounded" prophages: Hotspots for genetic renovation and innovation. Frontiers Genetics 10, 1-17 (2019). 
44. M. Jancheva, T. Böttcher, A Metabolite of Pseudomonas Triggers Prophage-Selective Lysogenic to Lytic Conversion in Staphylococcus aureus. J Am Chem Soc 143, 8344-8351 (2021).

45. J. E. Silpe, J. W. H. Wong, S. V. Owen, M. Baym, E. P. Balskus, The gut bacterial natural product colibactin triggers induction of latent viruses in diverse bacteria. bioRxiv [Preprint] (2021). https://doi.org/10.1101/2021.05.24.445430 (accessed 24 September 2021).

46. J. J. Dennehy, I. N. Wang, Factors influencing lysis time stochasticity in bacteriophage. BMC Microbiol 11, 174 (2011).

47. L. Imamovic, E. Ballesté, A. Martínez-Castillo, C. Garćia-Aljaro, M. Muniesa, Heterogeneity in phage induction enables the survival of the lysogenic population. Environ Microbiol 18, 957-969 (2016).

48. A. Melbinger, M. Vergassola, The Impact of Environmental Fluctuations on Evolutionary Fitness Functions. Sci Rep 5, 1-11 (2015).

49. L. S. Bittleston, M. Gralka, G. E. Leventhal, I. Mizrahi, O. X. Cordero, Context-dependent dynamics lead to the assembly of functionally distinct microbial communities. Nat Commun 11, 1-10 (2020).

50. B. B. Cael, K. Bisson, C. L. Follett, Can Rates of Ocean Primary Production and Biological Carbon Export Be Related Through Their Probability Distributions? Global Biogeochem Cy 32, 954-970 (2018).

51. E. J. Zakem, B. B. Cael, N. M. Levine, A unified theory for organic matter accumulation. Proc National Acad Sci 118, e2016896118 (2021).

52. E. Limpert, W. A. Stahel, M. Abbt, Log-normal Distributions across the Sciences: Keys and Clues. BioScience 51, 341-352 (2001).

53. A. M. Bolger, M. Lohse, B. Usadel, Trimmomatic: A flexible trimmer for Illumina sequence data. Bioinformatics 30, 2114-2120 (2014).

54. B. Bushnell, BBMap: A Fast, Accurate, Splice-Aware Aligner (2014).

55. S. I. Nikolenko, A. I. Korobeynikov, M. A. Alekseyev, BayesHammer: Bayesian clustering for error correction in single-cell sequencing. Bmc Genomics 14, S7 (2013). 
56. D. Li, et al., MEGAHIT v1.0: A fast and scalable metagenome assembler driven by advanced methodologies and community practices. Methods 102, 3-11 (2016).

57. Y. W. Wu, B. A. Simmons, S. W. Singer, MaxBin 2.0: An automated binning algorithm to recover genomes from multiple metagenomic datasets. Bioinformatics 32, 605-607 (2016). Methods 11, 1144-1146 (2014).

59. C. M. K. Sieber, et al., Recovery of genomes from metagenomes via a dereplication, aggregation and scoring strategy. Nat Microbiol 3, 836-843 (2018). Assessing the quality of microbial genomes recovered from isolates, single cells, and metagenomes. Genome Res 25, 1043-1055 (2015).

61. P. A. Chaumeil, A. J. Mussig, P. Hugenholtz, D. H. Parks, GTDB-Tk: A toolkit to classify genomes with the genome taxonomy database. Bioinformatics 36, 1925-1927 (2020).

62. G. E. Leventhal, et al., Strain-level diversity drives alternative community types in millimetre-scale granular biofilms. Nat Microbiol 3, 1295-1303 (2018).

63. A. R. Quinlan, I. M. Hall, BEDTools: A flexible suite of utilities for comparing genomic features. Bioinformatics 26, 841-842 (2010).

64. D. K. Button, B. R. Robertson, Determination of DNA Content of Aquatic Bacteria by Flow Cytometry. Appl Environ Microb 67, 1636-1645 (2001). 


\section{Figures}

655
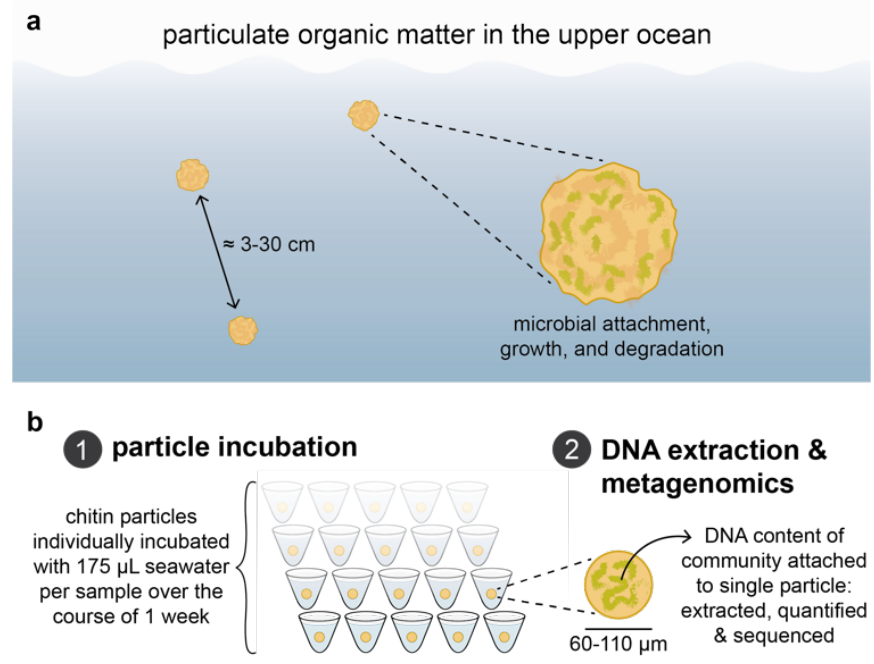

(3) MAG assembly, annotation, \& classification into roles

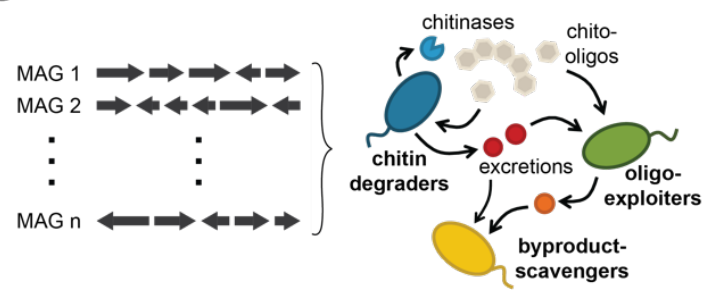

Figure 1. Modeling particulate organic matter degradation with a laboratory system of

658 enriching of marine microbes on chitin particles. (a) Microscale marine particles are spatially-

659 separated nutrient-rich habitats dynamically populated and degraded by complex communities of

660 heterotrophic bacteria. The interparticle distance range is estimated from data reported in Simon

661 et al. (16). (b) Schematic depicting experimental design and analysis. Microscale chitin particles

662 were individually incubated in seawater, and the DNA content of particle-attached communities

663 was quantified and submitted for shotgun metagenomic sequencing. Communities were

664 characterized using metagenome-assembled genomes (MAGs), which were classified into three

665 predicted ecological roles for this ecosystem: chitin degraders, chitooligosaccharide exploiters,

666 and metabolic byproduct scavengers. 


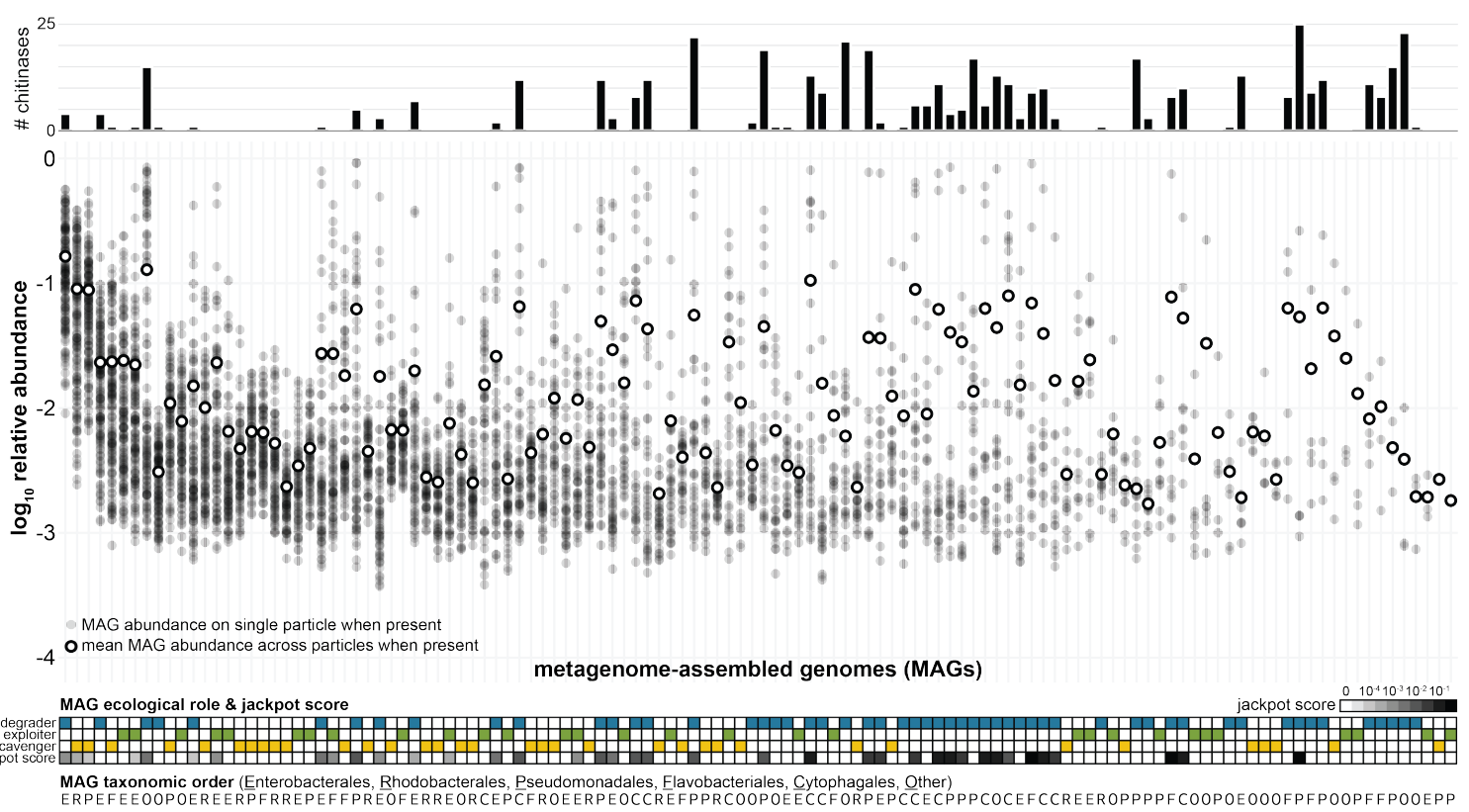

Figure 2. High compositional variability across replicate late-stage particles is driven by conditionally rare degrader taxa. Relative abundances of metagenome-assembled genomes

671 (MAGs; $n=120$ ) across late-stage particles. Smaller black dots indicate the relative abundance

672 of each MAG per particle $(n=149)$. Larger white dots indicate the $\log _{10}$ [mean relative abundance]

673 across the particles on which the MAG was found. MAGs are sorted from left to right by their

674 prevalence across particles (i.e. the number of particles on which they are detected). The bars

675 above show the number of chitinases encoded in each MAG. The annotations below show each

676 MAG's predicted ecological role (heatmap: blue = degrader, green = exploiter, yellow =

677 scavenger); jackpot score (heatmap: white = low, black = high); and taxonomic order $(\mathrm{E}=$

678 Enterobacterales, $\mathrm{R}=$ Rhodobacterales, $\mathrm{P}=$ Pseudomonadales, $\mathrm{F}=$ Flavobacteriales, $\mathrm{C}=$

679 Cytophagales, $\mathrm{O}=$ Other). See Fig. S1 for additional details. 
a

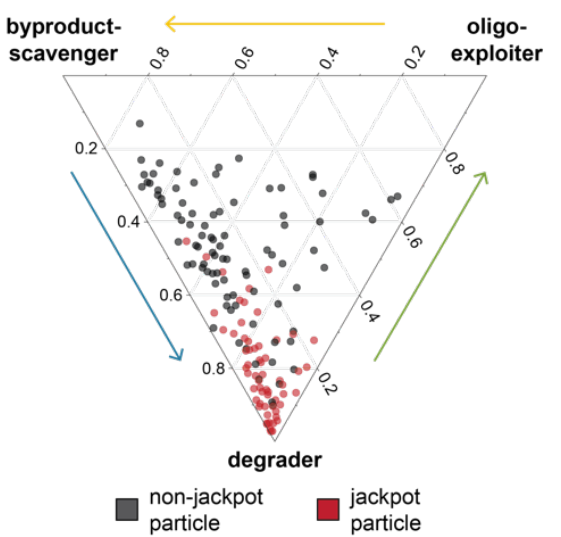

b

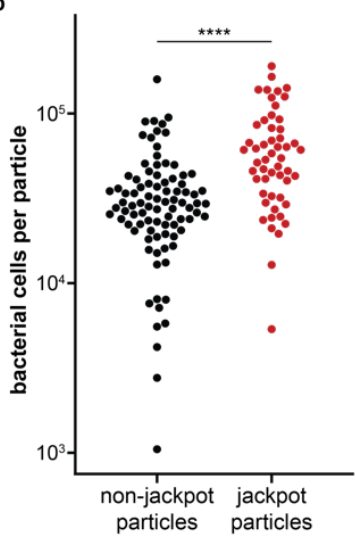

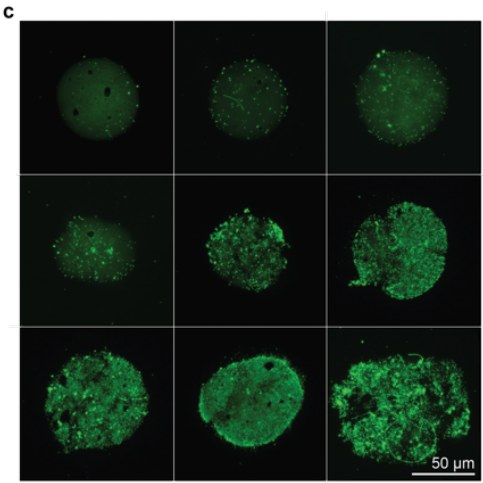

late-stage particle-attached communities

681 Figure 3. Late-stage particles diverge in community-level functional potential and biomass.

682 (a) Ternary plot of the relative abundances of organisms occupying the three ecological roles 683 (degrader, exploiter, scavenger) on each late-stage particle $(n=149)$, calculated by summing the 684 relative abundances of MAGs classified into each role. Red dots represent jackpot particles, and black ones represent non-jackpot particles. Jackpot particles harbored significantly higher degrader 686 populations than non-jackpot particles $(79.8 \%$ vs. $47.4 \%$ on average; Mann-Whitney $U$ test: $p<$ $6872.2 \times 10^{-16}$ ). (b) Estimates of absolute bacterial cell counts on late-stage particles through qPCR of 688 the 16S rRNA gene in DNA extracted from particle-attached communities. Jackpot particles (red 689 dots) harbored significantly higher numbers of cells (Mann-Whitney $U$ test: $p=2.3 \times 10^{-7}$ ) than non690 jackpot particles (black dots). (c) Representative images of late-stage particles that were harvested 691 after 167 hours of incubation in seawater and stained with the DNA-intercalating dye SYTO 9 (scale 692 bar, 50 $\mu$ m). Particle-attached communities spanned a range of growth states, from sparsely to densely populated. 
a
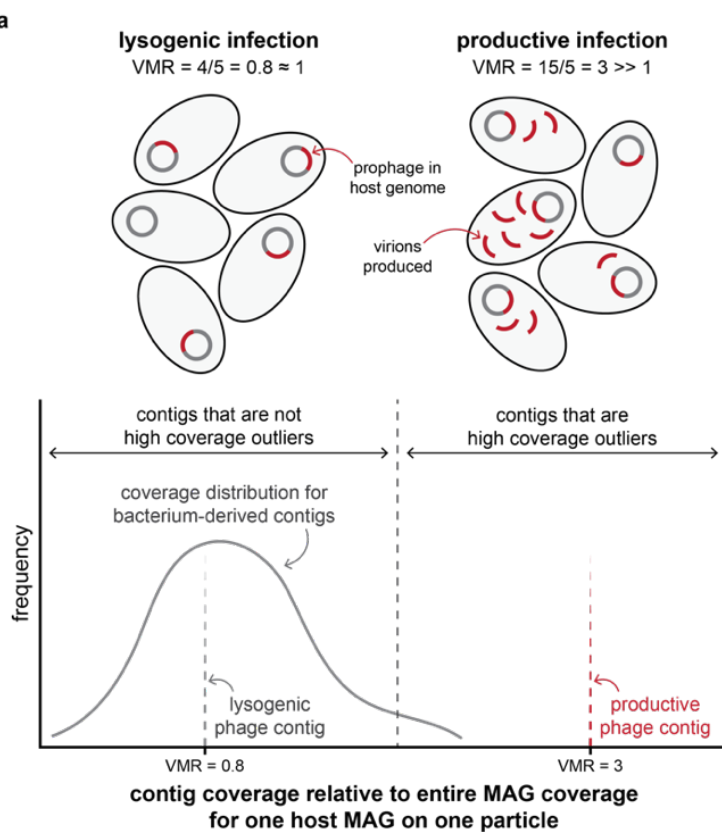

c

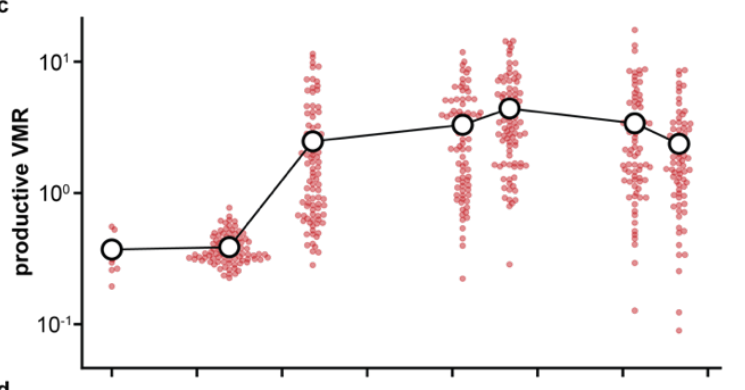

d

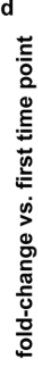

694

695

696

697

698

699

700

\section{b \\ e}

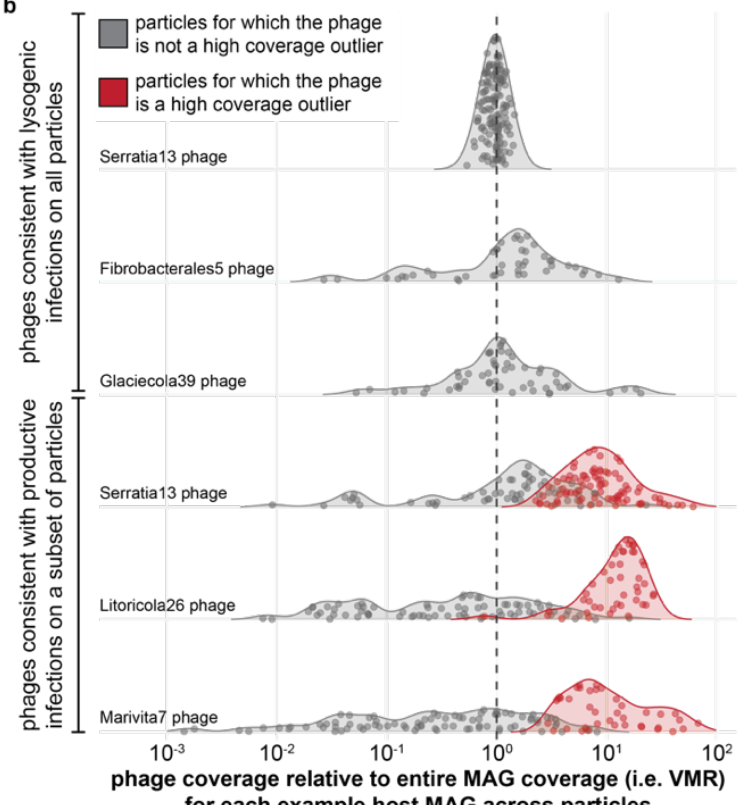
for each example host MAG across particles

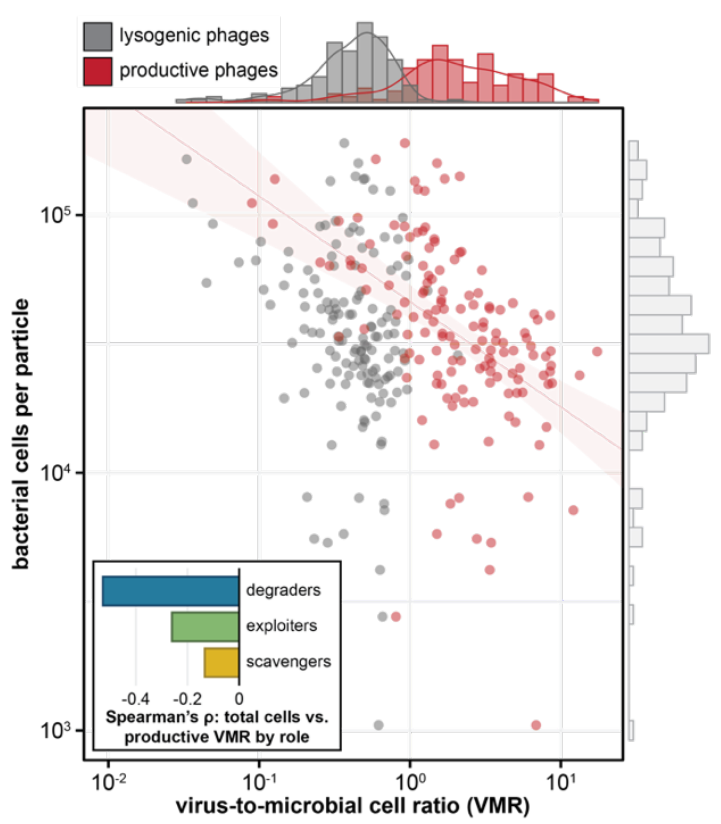

Figure 4. Bacteriophages become increasingly activated during community development and contribute to variability in bacterial abundances on late-stage particles. (a) Schematic of approach to detect productive phage infections from metagenomic data. Left: during lysogenic infections, prophages replicate with their bacterial hosts (virus-to-microbe ratio, VMR, $\approx 1$, top); lysogenic phage contigs have read coverage values similar to those of most bacterial contigs of their host MAG (bottom). Right: during productive infections, prophages replicate much more than 
701 their hosts (VMR >> 1, top); productive phage contigs have read coverage values much higher

702 than those of most bacterial contigs of their host MAG (bottom). (b) Representative examples of

703 phages with lysogenic coverage patterns on all late-stage particles (top three rows), and of

704 phages with productive coverage patterns on a subset of particles (bottom three rows). For each

705 phage contig, VMR is shown across late-stage particles on which each MAG is present. Gray

706 dots, particles on which the phage contig is not a coverage outlier; red dots, particles on which

707 the phage is a high coverage outlier. Dashed line: VMR $=1$. (c) Total VMRs for productive

708 phages over time. The first time point shows productive VMRs of initial seawater samples;

709 subsequent time points show productive VMRs for chitin particle-attached communities incubated

710 in seawater. Smaller red dots, values for individual samples; larger white dots, mean VMR for

711 each time point. (d) Metabolomic profiles of the seawater surrounding chitin particles as a

712 function of incubation duration. Values are depicted in terms of fold-change at each time point

713 relative to the first time point (dashed line: no change). Red line (and shading): mean ( \pm 1

714 standard deviation) weighted ion intensity (Methods). Blue line: number of unique metabolites. (e)

715 Main: Absolute bacterial cell counts on late-stage particles $(n=142)$, estimated through qPCR,

716 vs. each particle's total VMR for lysogenic phages (gray dots) and productive phages (red dots).

717 Cell counts were negatively correlated with productive VMRs (Spearman's $\rho=-0.56, p=3.3 \times 10^{-}$

$718{ }^{13}$; red line and shading: log-log linear regression and $95 \%$ confidence interval, $R^{2}=0.23, p=$

$\left.7191.3 \times 10^{-9}\right)$. Productive and lysogenic VMRs were decoupled (red vs. gray data: Spearman's $\rho=$

$7200.11, p=0.18)$. Marginal histograms: distributions of productive VMRs $($ red), lysogenic VMRs

721 (dark gray), and bacterial cell counts (light gray). Inset: Bar plot of values of Spearman's $\rho$

722 between cell counts and productive VMRs of bacterial populations by ecological role (blue =

723 degraders, green = exploiters, yellow = scavengers; see Fig. S14b for details). 


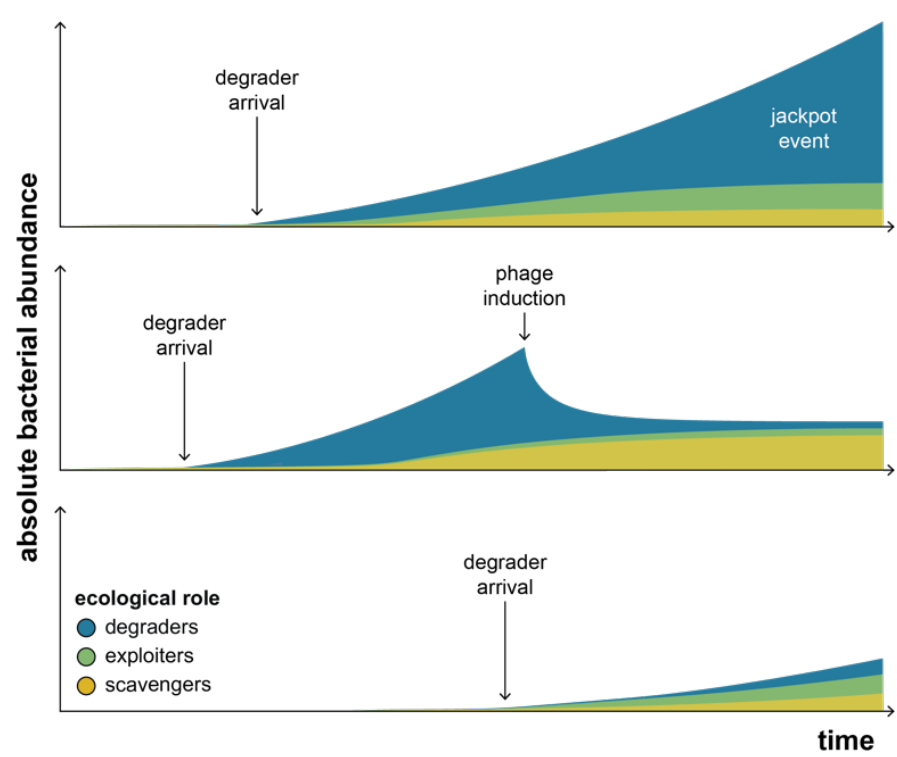

725 Figure 5. Conceptual model of key processes contributing to the diversification of

726

communities on microscale particles. Schematics of community development over time are

727 shown for three example particles, with the absolute abundances depicted for bacterial

728 populations by ecological role (blue $=$ degraders, green $=$ exploiters, yellow $=$ scavengers). Based

729 on our conceptual model (see Discussion), high-biomass jackpot particles are those on which

730 degraders arrive early and resist phage induction, leading to high relative and absolute degrader

731 abundances (top). By contrast, low-biomass particles are those on which degraders are not able

732 to proliferate, either because phage induction leads to their population collapse (middle) or

733 because they become established on a particle relatively late (bottom). 


\section{Supplementary Information Text}

735

\section{Models of abundance fluctuations}

\section{$738 \quad$ Notation and context}

740 We considered a system with $M$ MAGs and $P$ particles. Let $x_{i}$ be the abundance of MAG $i$ on a

741 particle and $X=\sum_{i} x_{i}$ be the total abundance. The probability distribution $p(\underline{x})$ is the probability of

742 observing a given vector of abundance $\underline{x}$, while $p_{i}(x)$ is the probability that species $i$ has abundance

$743 x$ and $P(X)$ is the probability that the total abundance is $X$. We also define $y_{i}=\log x_{i}$ and $Y=\log X$

744 ( $\log$ means natural log everywhere).

746 Models \#1-3 are reasonable models that nevertheless do not recapitulate the observed trends (i.e.

747 the right-skewed distributions of relative taxon abundances [Fig. S2] and the left-skewed 748 distribution of absolute cell abundances [Fig. 3b]), which model \#4 (referenced in the main text) 749 does reproduce.

\section{Model \#1: Stochastic arrival and exponential growth}

We assume that MAGs arrive stochastically to a particle and grow exponentially with a fixed MAGspecific growth rate $r_{i}$. The log-abundance of MAG $i$ at time $t$ will therefore be $\left.y_{i}=r_{i}\left(t-t_{i}^{a}\right)\right)$, where $t_{i}^{a}$ is the arrival time of MAG $i$.

757 The only source of variation across particles is the intrinsic randomness in the arrival time, which

758 is exponentially distributed with (migration) rate $\lambda_{i}$. If we are considering only particles where $i$ is 759 present, the probability should be normalized between 0 and the duration of the experiment $t$, which 760 leads to 


$$
\rho_{i}\left(t^{a}\right)=\frac{\lambda_{d} e^{-\lambda_{i} t^{a}}}{1-e^{-\lambda_{i} t}}
$$

763

764

One can obtain the probability of observing a MAG with log-abundance $y_{i}$ at time $t$ simply by

765 inverting the relationship $\left.y_{i}=r_{i}\left(t-t_{i}^{a}\right)\right)$ :

766

$$
p_{i}(y)=\frac{\lambda_{i}}{r_{i}} \frac{\exp \left(-\lambda_{i} t+\lambda_{i} \frac{y}{r_{i}}\right)}{1-e^{-\lambda_{i} t}}
$$

768

and, therefore, the probability of the abundance (conditioned on being present) reads

$$
p_{i}(x)=\frac{\lambda_{i}}{r_{i}} \frac{\exp \left(-\lambda_{i} t\right)}{1-e^{-\lambda_{i} t}} x^{\frac{\lambda_{i}}{r_{i}}-1}
$$

772

773 Note that this distribution is normalized between 0 and $\tilde{x}_{i}=e^{r_{i} t}$. We can therefore rewrite this expression as

$$
p_{i}(x)=\frac{\lambda_{i}}{r_{i}} \tilde{x}_{i}^{\frac{\lambda_{i}}{r_{i}}} x^{\frac{\lambda_{i}}{r_{i}}-1} .
$$

Both the arrival rate $\lambda_{i}$ and the growth rate $m_{i}$ differ across MAGs. We set their values by drawing them from two independent lognormal distributions. In particular, each $\lambda_{i}$ for $i=1, \ldots, M$ was drawn from a lognormal distribution with mean $\bar{\lambda}$ and log-variance $s_{\lambda}^{2}$. Similarly, each $r_{i}$ was drawn from a lognormal with mean $\bar{r}$ and variance $s_{r}^{2}$.

783 Fig. S16 shows the distribution of collapsed MAG relative abundances and the distribution of total abundances obtained with this model. Model \#1 always predicts a relative log-abundance

785 distribution with negative skewness and a total log-abundance distribution with non-negative 786 skewness (contrarily to what observed in the data; see Fig. 3b). 
790 Model \#2 assumes that MAGs arrive on particles with rate $\lambda_{i}$. The population growth that follows is 791 determined by a birth-death process with constant per-capita birth and death rates $\left(b_{i}\right.$ and $d_{i}$, respectively). The (average) growth rate $r_{i}$ equals $b_{i}-d_{i}$.

794 Similar to the procedure of model \#1, we assumed that the values of migration, growth, and death rates of each MAG were initialized as lognormal random variables with means $\bar{\lambda}, \bar{r}$, and $\bar{d}$ and logvariances $s_{\lambda}^{2}, s_{r}^{2}$, and $s_{d}^{2}$.

Fig. S17 shows that model \#2 always predicts a total log-abundance distribution with positive skewness, therefore failing in reproducing the empirical shape of the total abundance distribution.

\section{Model \#3: Stochastic arrival and exponential growth with environmental stochasticity}

Model \#3, similarly to model \#1, assumes that MAGs arrive stochastically to a particle with arrival 804 rate $\lambda_{i}$ and then grow exponentially. When a MAG arrives on a particle, it starts growing exponentially. The growth rate of MAG $i$ is not fixed, equal to $r_{i}$ across all particles, but is itself a random variable. In particular, the growth rates of MAG $i$ across particles are normally distributed with mean $r_{i}$ and variance $\sigma_{i}^{2}$ proportional to the mean squared: $\sigma_{i}^{2}=c_{r}^{2} r_{i}^{2}$, where $c_{r}$ is the coefficient of variation. means $\bar{\lambda}$ and $\bar{r}$ and log-variances $s_{\lambda}^{2}$ and $s_{r}^{2}$.

813 Fig. S18 shows that model \#3 always predicts a total log-abundance distribution with positive 814 skewness, therefore failing in reproducing the empirical shape of the total abundance distribution. 
816 Model \#4: Exponential growth with environmental stochasticity conditioned on degrader

\section{7 presence}

819 In the previous models, the growth of all MAGs was only conditioned on arrival. This assumption

820 inevitably led to total log-abundance distributions with positive skewness, contrarily to the empirical 821 observation of negative skewness.

823 Model \#4 assumes that, for a particle to become viable for growth, the presence of a degrader MAG

824 is required first. The arrival rate of a degrader is $\lambda^{d}$. All the cells that arrive to the particle after the 825 first arrival of the degrader are able to grow. The time at which the population of MAG $i$ on a particle 826 will start to grow will be $t_{g}^{i}=t_{a}^{i}+t_{d}$, where $t_{d}$ is the time of arrival of the degrader (an exponential 827 random variable with rate $\lambda^{d}$ ) and $t_{a}^{i}$ is the time between arrival of the degrader and the arrival of 828 the MAG $i$ (an exponential random variable with rate $\lambda_{i}$ ).

830 Starting at $t_{g}^{i}$, MAG $i$ will start to grow exponentially with a random, normally distributed, growth 831 rate with mean $r_{i}$ and coefficient of variation $c_{r}$. Similar to the previous models, the arrival rate $\lambda_{i}$ 832 and the average growth rates $r_{i}$ are lognormally distributed with means $\bar{\lambda}$ and $\bar{r}$ and log-variances $833 s_{\lambda}^{2}$ and $s_{r}^{2}$.

835 Fig. S19 shows that the predictions of model \#4 agrees with the empirical observations. The total 836 log-abundance distribution has a negative skewness, while the distribution of MAG relative 837 abundances has a positive skewness. The shape of the patterns is robust across different 838 parameters values. Only when the variation across MAGs is comparable to the growth rate 839 fluctuations across particles $\left(c_{r} \sim s_{r} \sim 1\right)$ does the total log-abundance distribution display a 840 positive skewness. 


\section{Extended methods}

845 Sample collection and incubation with individual chitin particles

Seawater sampling and treatment. Nearshore coastal ocean surface water samples were collected on July 15, 2017 from Canoe Beach, Nahant, MA, USA (42²5'11.5” N, 7054'26.0" W). The seawater was immediately transported to Parsons Laboratory (MIT, Cambridge, MA, USA) for processing. In order to decrease the degree of dissimilarity between seawater aliquots used in incubations with chitin particles, large particulate matter was removed (using a $63 \mu \mathrm{m}$ filter), and the flow-through was concentrated via gentle centrifugation in $1 \mathrm{~L}$ batches at $4000 \times$ $g$ for 5 minutes. The lower $100 \mathrm{~mL}$ of each $1 \mathrm{~L}$ batch was saved and pooled; aliquots of this water in $175 \mu \mathrm{L}$ volumes were either used for particle incubations or stored at $-20^{\circ} \mathrm{C}$ for downstream DNA extraction and metagenomic sequencing.

Seawater incubation with individual chitin particles. Artificial seawater (ASW), used for washing and storing chitin particles, was prepared by dissolving $40 \mathrm{~g} / \mathrm{L}$ sea salts (SigmaAldrich, \#S9883) in Milli-Q deionized water and filtering the solution through a 0.22- $\mu \mathrm{m}$ filter. Chitin magnetic particles (New England Biolabs, \#E8036L) stored in $20 \%$ ethanol were washed three times ( $2 \mathrm{~mL}$ particles resuspended in $50 \mathrm{~mL}$ ASW) using a magnet to pull down the particles. Aliquots of washed chitin particles were further diluted in ASW in sterile petri dishes and individually selected beneath a dissecting microscope in a laminar flow hood. Single chitin particles were transferred in $3 \mu \mathrm{L}$ volumes of ASW into the wells of 96-well plates (Thermo Fisher, \#AB0600L; UV-sterilized; free from DNase, RNase, and human DNA), with one chitin particle per well. The individual particles selected had a diameter of $85.0 \pm 24.0 \mu \mathrm{m}$, which was quantified from a set of 60 particles on an ImageXpress Micro Confocal (Molecular Devices). Plates containing individual particles were stored at $4^{\circ} \mathrm{C}$ until they were inoculated consecutively with $175 \mu \mathrm{L}$ of filtered, centrifuged seawater per well. The plates were sealed 
870

871

872

873

874

875

876

877

(VWR, \#89092-056) and rotated end-over-end at 7.5 revolutions/minute at room temperature. The particles in an entire plate were harvested at each time point (after 12, 22.75, 34.5, 46, 59, $69,82,92,103,116.75,113,153.5$, and 166.5 hours of incubation) by pipetting the contents of each well onto a sterile petri dish and inspecting the water under a dissecting microscope in a laminar flow hood. Each particle was transferred in $1 \mu \mathrm{L}$ volumes into 96-well plates (Thermo Fisher, \#AB0600L) pre-filled with $100 \mu \mathrm{L}$ of TE buffer; plates with harvested particles were stored at $-20^{\circ} \mathrm{C}$ until downstream processing. The seawater surrounding each harvested particle was also saved in 96-well plates (Thermo Fisher, \#AB0600L) and stored at $-20^{\circ} \mathrm{C}$ until downstream processing.

DNA extraction and metagenomic sequencing. DNA extractions were performed for twelve $175 \mu \mathrm{L}$-volume aliquots of the initial, unincubated seawater, as well as for particles harvested after $34.5,59,103,116.75,113,153.5$, and 166.5 hours of incubation. DNA was extracted from all samples with the Agencourt DNAdvance Genomic DNA Isolation Kit (Beckman Coulter) using reagent volumes $0.5 \mathrm{X}$ relative to those specified in the manufacturer's protocol, except for the elution buffer, of which only $30 \mu \mathrm{L}$ was used for each sample to avoid over-diluting low DNA yields. Metagenomic libraries were prepared with the Nextera XT DNA Library Prep Kit and index primers (Illumina) using the protocol developed by Rinke et al. (1) for low DNA input samples. While the results from the protocol in Rinke et al. were reproducible with as little as $100 \mathrm{fg}$ of input DNA, the authors recommend using a minimum of $1 \mathrm{pg}$ as input. Based on our qPCR measurements of DNA extracted from individual particle-attached communities (as described in the Methods section "Cell count estimation"), only one of our libraries (with 0.44pg input) was created with less than $1 \mathrm{pg}$ DNA. The modifications to the manufacturer's library preparation protocol included (i) diluting the Amplicon Tagment Mix 1:10 in non-DEPC-treated nuclease-free water, and (ii) increasing the number of PCR amplification cycles of the tagmented DNA from 12 to 20 cycles. Amplified libraries were purified with 0.6X AMPure XP beads. Each library was quantified on an Agilent 4200 TapeStation system with High Sensitivity D5000 ScreenTapes (Agilent Technologies) following the manufacturer's protocol, and successfully amplified libraries were pooled by time point in equimolar 
amounts. Sequencing was performed on an Illumina HiSeq 2500 machine (250bp paired-end reads) at the Genome Technology Core of the Whitehead Institute for Biomedical Research (MIT,

900 Cambridge, MA, USA). See Table S5 for all sample metadata.

\section{Metagenomic analyses}

903

Read pre-processing. Raw sequencing reads were clipped (to remove adapter sequences) and trimmed for quality with Trimmomatic v0.36 (2) (parameters: LEADING:3, TRAILING:3, SLIDINGWINDOW:10:20, MINLEN:36). Reads mapping to the PhiX genome were filtered out with BBDuk v38.16 (3) (parameters: $k=31$, hdist=1) and those mapping to the human genome (masked by Brian Bushnell at the Joint Genome Institute to prevent false positives) were identified and removed using BBMap v38.16 (parameters: minid=0.95 maxindel=3 bwr=0.16 $\mathrm{bw}=12 \quad$ minhits $=2$ qtrim=rl trimq $=10$ untrim; reference genome: hg19_main_mask_ribo_animal_allplant_allfungus.fa.gz). were used for all tools unless otherwise specified. Trimmed, filtered reads were error-corrected using BayesHammer (4) (a component of the SPAdes v3.13.0 pipeline) in order to improve contig assembly. Reads within each time point were pooled and co-assembled using MEGAHIT v1.2.9 (5). Assembled contigs at least $1 \mathrm{~kb}$ in length were binned using two complementary tools - MaxBin v2.2.7 (6) and CONCOCT v1.1.0 (7). To provide CONCOCT with coverage estimates, error-corrected reads were mapped to contigs using Bowtie 2 v2.3.4.1 (8) with the parameters and approach described in Leventhal et al. (9). Bins generated with MaxBin and CONCOCT were consolidated and filtered using DAS Tool v1.1.1 (10) and evaluated for completeness and contamination with CheckM v1.1.2 (11). The resulting 251 bins that were at least $50 \%$ complete and at most $10 \%$ contaminated were used as reference MAGs, with median completeness and contamination values of $93.7 \%$ and $3.9 \%$, respectively, across this set of MAGs (Table S1). Taxonomic classifications from the Genome Taxonomy Database 

from separate co-assemblies were identified and clustered through a pipeline developed by Dr. Jakob Russel for performing whole-genome comparisons of each MAG against all others with BLAT v36x2 (14). Briefly, a similarity score was calculated for each MAG relative to another by dividing the combined length of its contigs at least $98 \%$ identical to those in the compared MAG by the combined length of all its contigs. A threshold for distinguishing high similarity scores from low ones was determined using Otsu's method (15) with code derived from the R (16)

MAG ecological role assignments. For each MAG, protein-coding genes were predicted and package EBImage (17). 132 clusters of MAGs with mutually high similarity scores were identified, and all MAGs in each cluster had consistent GTDB-based taxonomic assignments. For one MAG cluster, one of the MAGs was classified as a different genus from the other MAGs; this MAG was separated from the cluster. We chose to consider clustered MAGs as a unit, rather than to dereplicate them, in order to retain potential strain-level microdiversity in our reference set. translated using Prodigal v2.6.3 (18). Predicted protein sequences were compared to a custom database of profile hidden Markov models (HMMs) of proteins involved in growth on chitin using the hmmsearch function of HMMER v3.3 with default parameters (19). Publicly-available HMMs were downloaded from the Pfam v33.1 (20) or TIGRFAM v15.0 databases (21) (see Table S6 for accession numbers). Custom HMMs were made by identifying experimentallyverified proteins of interest $(22,23)$, finding their homologs in the UniProtKB/Swiss-Prot v2020_06 database (24), creating a seed alignment using MAFFT v7 with default parameters $(25,26)$, and building the profile HMMs using the hmmbuild function of HMMER with default parameters (see Table S6 for details on each custom HMM). Protein-coding sequences were annotated based on the hmmsearch results if the protein length was at least 100 amino acids, the independent E-value was less than $1 \times 10^{-9}$, and the domain score was greater than 30 . Only the most significant annotation was used for each protein sequence. Gene copy numbers were calculated for each MAG by tallying the number of annotations made for each protein group 
(Table S1). Ecological roles (as degraders, chitooligosaccharide exploiters, or metabolic byproduct scavengers) for MAGs were defined based on the gene content patterns observed for strains previously isolated from particle enrichments $(27,28)$, fully sequenced, and phenotyped according to their abilities to grow on colloidal chitin, chitobiose, and GIcNAc (29). MAGs were classified as degrader genomes if they encoded at least 1 chitinase and at least 1 copy of any of the following genes: GlcNAc-specific methyl-accepting chemotaxis protein (MCP), GlcNAc-specific phosphotransferase system IIBC component (PTS), GlcNAc-specific TonB-dependent transporter (TBDT), N,N'-diacetylchitobiose phosphorylase, beta- $\mathrm{N}$ acetylhexosaminidase, or GIcNAc kinase. MAGs were classified as exploiter genomes if they encoded 0 chitinases and had at least one of the following characteristics: more than 1 copy of beta-N-acetylhexosaminidase or at least 1 copy of MCP, PTS, TBDT, or N,N'diacetylchitobiose phosphorylase. MAGs were classified as scavenger genomes if they encoded 0 chitinase, MCP, PTS, TBDT, and N,N'-diacetylchitobiose phosphorylase copies, and 1 or fewer copies of beta-N-acetylhexosaminidase. If MAGs clustered by similarity were assigned different ecological roles by these heuristics, then either (i) the role assigned to all MAGs defaulted to the role of the MAG with the lowest contamination and/or highest completeness (which occurred for 4 MAG clusters), or (ii) the MAG cluster was split into two subclusters (which occurred for 5 MAG clusters); these discrepancies are indicated in Table S1. Following this MAG cluster curation, there were a total of 138 MAG clusters.

Read mapping to MAGs for relative abundance estimation. All trimmed, filtered reads were mapped competitively against the MAGs created from sequencing particle-attached communities; the initial, unincubated seawater; and the negative controls (see the Methods section "Mock communities and negative controls"). Samples with fewer than 100,000 trimmed, filtered reads were excluded from analyses. Read mapping was performed using Bowtie 2 v2.3.4.1 (8) with the parameters and approach described in Leventhal et al. (9) and postprocessed using SAMtools v1.7 (30). Reads that best mapped (based on alignment scores) to MAGs obtained from the negative controls (which were contaminants from laboratory reagents) 
and to MAGs obtained from particle sequences that were also likely environmental contaminants (indicated in Table S1; determined through a literature search of each strain's taxonomy in studies of the marine environment) were removed from consideration when estimating community compositions. To avoid artifactually double-counting hits from paired reads, only the best hit of the forward read was considered for read pairs that survived trimming and quality filtering. Hits to completely bacteriophage-derived contigs (as opposed to prophages integrated into bacterial genome contigs) were also excluded from estimates of MAG relative abundances (see the Methods section "Bacteriophage analysis"). To minimize spurious detection, MAGs were considered to be "present" in a sample if they recruited at least $0.05 \%$ of the reads in a sample; for MAGs that recruited reads below this threshold in a sample, their abundance was set to 0 for that sample. MAG relative abundances for MAGs above this threshold were calculated for each sample by (1) tallying the hits to all MAGs in each MAG cluster; (2) normalizing the tally by the average genome length of all MAGs in each MAG cluster; and (3) dividing the normalized tallies for each MAG cluster by their sum for each sample. Therefore, for MAGs clustered together based on similarity (see the Methods section "Metagenome assembly, binning, and MAG taxonomic assignment"), their relative abundances are represented in that of the entire MAG cluster to which they belong; this calculation circumvents the artificial underestimation of MAG relative abundances that would otherwise be obtained with a non-dereplicated reference set. The relative abundances of organisms occupying the three ecological roles (degrader, exploiter, scavenger) on each particle were calculated by summing the relative abundances of MAGs classified into each role. Based on information gathered from relative abundance estimation, particles harvested at 113 hours post-inoculation were excluded from analyses because of a clear batch effect at that time point characterized by high abundances of MAG Serratia_liquefaciens93 (96.7\% of particles on which Serratia_liquefaciens 93 was at least $10 \%$ abundant were from $t=113 \mathrm{~h}$, which included $98.9 \%$ of particles from that time point; this MAG was also the most abundant MAG on $81.1 \%$ of particles from $t=113 \mathrm{~h}$ and was not the most abundant MAG on any particles from other time points; see Table S7). 

variability was estimated as the Aitchison distance between the community compositions of 1013 pairs of samples (i.e. the Euclidian distance between center log-ratio-transformed MAG relative abundance vectors). Aitchison distances were calculated between aliquots of the initial, unincubated seawater and between late-stage particle communities separately, and the 1016 distributions of distances between all pairs of samples were compared to each other.

Definitions of jackpot MAGs and jackpot particles. A jackpot score was calculated for each MAG cluster to quantitatively reflect the properties of rarity across most particles and dominance on a few particles. Based on relative abundances across late-stage particles, each MAG's jackpot score was defined as: with low scores do not. The jackpot score for each particle was calculated as the weighted average of MAG jackpot scores (i.e. the sum of the relative abundance of each MAG cluster multiplied by its jackpot score). Each particle's jackpot score was compared to its species evenness (calculated as Pielou's evenness, i.e. the Shannon diversity index divided by the natural logarithm of species richness) with the expectation that particles that most strongly display the jackpot phenomenon have low species evenness. Particles were defined as "jackpot particles" if they have jackpot scores that exceed the threshold value above which logtransformed values of species evenness drop sharply (Fig. S5a); this value corresponds to the $60^{\text {th }}$ percentile of jackpot particle scores. For comparing the binary categories of "jackpot degraders" and "non-jackpot degraders" (Fig. S15a), "jackpot degraders" were those MAGs that had jackpot scores greater than zero and that were present on less than $75 \%$ of late-stage particles; this thresholding was done in order to exclude the MAG clusters Serratia13 and 
1038 Fibrobacterales5 that had very low yet non-zero jackpot scores because of their high relative 1039 abundances on many particles (see Tables S1 and S7). particles by the MAG abundances theoretically obtained by sequencing particles in bulk.

1043 To evaluate the extent to which community compositions at the single particle level diverged 1044 from that of a "bulk" measurement theoretically obtained by sequencing all particles together, 1045 we calculated "bulk" MAG abundances by (1) normalizing the mapped read counts to each 1046 MAG cluster by the total number of read counts for each sample; (2) summing the counts for 1047 each MAG cluster across samples; (3) normalizing the sum across samples by the average 1048 genome length of all MAGs in each MAG cluster; and (4) dividing the length-normalized counts 1049 for each MAG cluster by their sum. (These "bulk" MAG relative abundances are equivalent to 1050 the mean MAG relative abundances calculated across all particles, including those particles 1051 where MAGs are absent.) The percent variance in the abundance ranks of MAGs on single particles explained by the abundance ranks for the theoretical bulk measurement was calculated for each particle as the square of the Pearson correlation coefficient (between each 1054 individual vs. the bulk abundance rank), multiplied by 100.

Multivariate analysis. We inferred the number of conditional dependencies between MAGs from the estimated inverse covariance matrix of center log-ratio-transformed MAG relative abundances, repeating this process for 1000 randomizations of the data in which we permuted particle labels for each MAG but retained their abundance distributions. The inverse covariance matrices were estimated using a graphical lasso approach with the R package glasso (31) for several values of the regularization parameter $(\rho=0.005, \rho=0.001, \rho=0.0005$, and $\rho=$ 0.0001). 
1066 degrader population relative abundances in particle-attached communities, reads were also

1067 mapped to a reference set of chitinase genes (regardless of binning). All assembled contigs

1068 (binned and unbinned) were annotated for chitinase genes using the HMM-based approach

1069 described in the Methods section "MAG ecological role assignments." A custom DIAMOND

1070 database of 3,370 translated chitinase genes was created using the makedb function of

1071 DIAMOND v0.9.10.111 (32) with default parameters. Because of the high sequence diversity

1072 of chitinase genes, we chose to make this custom database so that the chitinase sequences

1073 used as references would be representative of those found in this experiment. Trimmed,

1074 quality-filtered reads were mapped to this database using the blastx function of DIAMOND with

1075 default parameters. To avoid artifactually double-counting hits from paired reads, only the best

1076 hit of the forward read was considered for read pairs that survived trimming and quality filtering.

1077 Only the most significant hit was counted for each read and only if the E-value was less than

1078 or equal to $1 \times 10^{-25}$. The number of such hits was tallied for each sample and divided by the

1079 number of trimmed, quality-filtered reads used in the mapping step to yield the percent of reads

1080 in each sample mapping to chitinase genes (Table S5). If the degrader population relative

1081 abundance estimated by MAGs were a consistent approximation of the true degrader

1082 population abundance, then the wide range in the number of chitinases encoded in each

1083 degrader MAG (Table S1) would be reflected in the percent of reads in each community 1084 mapping to chitinase genes. Therefore, the community-weighted mean (CWM) for chitinases 1085 was calculated as another comparison to the percent of reads mapping to chitinases. The 1086 chitinase CWM was calculated by multiplying the relative abundance of each degrader MAG 1087 (or MAG cluster) by the number of chitinases encoded in it (or the mean number of chitinases 1088 for a MAG cluster), and finally by summing these values.

\section{Bacteriophage analysis.}



0735191, DBI-1265383, DBI-1743442) using its RefSeqABVir database and default parameters; and (ii) VirFinder v1.1 (34) with default parameters. Contigs were classified as phage-derived if they met one of the following standards as employed in Gregory et al. (35): (i) they were classified by VirSorter as Category 1 or 2 (complete phage contig, higher confidence); (ii) they were classified by VirFinder with a score $\geq 0.9$ and $p$-value $<0.05$; or (iii) they were classified both by VirSorter as Category 3 (complete phage contig, lower confidence) and by VirFinder with a score $\geq 0.7$ and $p$-value $<0.05$.

Identifying productive vs. Iysogenic phage-derived contigs. We used a read coveragebased approach to categorize phage-derived contigs as productive or lysogenic in particleattached communities. Phages in a productive cycle in a particular sample would have a higher coverage than the bacterial contigs of the MAG with which they were binned because of the multiple virion copies produced per bacterial cell. In contrast, phages in a lysogenic cycle would have coverage values comparable to those of the bacterial contigs of the MAG with which they were binned. We reasoned that contigs classified as phagederived, especially those belonging to the genomes of temperate phages, were likely to be binned into the MAGs of their bacterial hosts because: i) phage $k$-mer signatures tend to be more similar to those of their specific hosts than to those of random bacteria $(34,36$, 37); ii) phages in a lysogenic cycle will have the same sequencing read coverage patterns as their hosts across samples; and iii) accordingly, both of the binning algorithms we employed clustered contigs based on their tetranucleotide frequencies and their coverage levels across multiple samples. Because our approach relied on comparisons between cobinned phages and MAGs, we considered in our analyses only phage-classified contigs at least $5 \mathrm{~kb}$ in length, since the likelihood of mis-binning decreases with increasing contig length. 

MAGs for relative abundance estimation"), per-base coverage values for all binned contigs were computed with the genomecov function of BEDTools v2.27.0 (38) and were used to calculate contig-wide average coverage values. For each MAG and for each sample, a phage-derived contig was considered to be productive if its coverage was greater than the coverage of the $95^{\text {th }}$ percentile bacteria-derived contig in the same MAG. A phage derivedcontig was considered to be lysogenic in a sample if its coverage did not exceed the coverage of the $95^{\text {th }}$ percentile bacteria-derived contig in the same MAG. Through this pipeline, we identified 263 phage contigs with coverage patterns consistent with productive infections in a subset of samples and 256 phage contigs with coverage patterns consistent with lysogenic infections in all samples (Table S2). one sample is defined as the phage contig coverage divided by average coverage of the MAG with which it is binned (which was calculated using only the bacteria-derived contigs). Total VMRs - i.e. the total number of phage copies relative to the total number of bacterial MAG copies in an entire sample - were calculated separately for productive and lysogenic phage contigs. The total productive VMR for a sample was defined as: 


$$
=\frac{\text { total } \# \text { phage copies (due to lysogenic infections) }}{\text { total \# bacterial genome copies }}
$$

where $n$ is the number of MAGs found in a sample. VMRs for each ecological role (i.e. for the subpopulation in a community that belongs to one of the three roles of degrader, exploiter, or scavenger) were calculated using the same formulas as above while considering only the MAGs of each role and their associated phages. When calculating total VMRs, we used the average coverage value of all phage contigs in each MAG (rather than the sum of the coverage values for all phage contigs in each MAG) to obtain a more conservative estimate of phage copy number. For example, if two phage contigs belonged to the same phage genome but did not overlap in sequence, they would appear to be two separate phages; thus, using their sum would double the apparent phage copy number, conceivable that phage contigs identified as "productive" have high coverage relative to their associated bacterial MAGs simply due to sequencing noise. We performed two 
are identified based on coverage, there is a chance that more productive phages would be found in samples with more reads. (Ensuring that this is not the case is one of the controls used in Kieft et al. (39), which also employs a coverage-based method for finding productive phages in mixed metagenomes.) Therefore, we calculated the Spearman's correlation coefficient between the number of reads in a sample and the number of phagederived contigs with coverage values above the $95^{\text {th }}$ percentile for their MAG (as described in the Methods section "Identifying productive vs. lysogenic phage-derived contigs"). We calculated these correlations for samples within time points to avoid spurious correlations created by systematic differences in the number of reads obtained across time points. The phage contigs from the MAGs that showed a significant correlation $(p<0.05)$ were excluded from analyses. Secondly, for each sample, we compared the average coverage of all phage-derived contigs ( $\geq 5 \mathrm{~kb}$ ) with coverage values above the $95^{\text {th }}$ percentile for their MAG with the average coverage of all bacteria-derived contigs ( $\geq 5 \mathrm{~kb}$ ) with coverage values above the $95^{\text {th }}$ percentile for their MAG. If the high coverage phage contigs have comparable average coverage to the bacterial contigs, that would indicate that the phage contigs had high coverage only due to sequencing noise. The average bacterial coverage is larger than the average phage coverage in only $2.0 \%$ (3/149) of late-stage particles (Fig. S12). Therefore, for samples with high total productive VMRs, phage contigs with high coverage values likely represent phages that were replicating more than their bacterial hosts, rather than representing contigs with randomly higher coverage values.

1195 using seawater from the same location sampled for this project (27). The total genomic DNA of

1196 Vibrio splendidus strain 1A01 (BioProject \#PRJNA414740, Accession \#PDUR00000000) and

1197 Maribacter sp. 6B07 (BioProject \#PRJNA414740, Accession \#PDUT00000000) was extracted 1198 using the MasterPure DNA Purification Kit (Epicentre), and double-stranded DNA content was 
1199 quantified using the Quant-iT PicoGreen dsDNA Assay Kit (Invitrogen). The DNA of each strain 1200 was mixed in equimolar amounts and serially diluted to either $50 \mathrm{pg}$ or $5 \mathrm{pg}$ total (to include a range 1201 of expected DNA input concentrations from extractions of communities attached to single chitin 1202 particles). Metagenomic libraries from three technical replicates of each concentration, as well as 1203 from six negative controls (containing only nuclease-free water), were prepared using the same 1204 protocol used for individual chitin particle-attached communities (as described in the Methods 1205 section "DNA extraction and metagenomic sequencing"). The results from the mock community 1206 sequencing are shown in Fig. S4. Of the six negative control libraries, only three amplified; the 1207 MAGs recovered from these samples included Delftia acidivorans and a Brevundimonas sp., which 1208 belong to taxonomic groups previously found as contaminants in laboratory reagents used in DNA 1209 extractions and sequencing $(40,41)$. These MAGs were included as references for the 1210 metagenomic analysis, and the reads best mapping to them (based on alignment scores) were 1211 removed from consideration when estimating community compositions.

1213 Cell count estimation. Bacterial DNA extracted from individual particle-attached communities was 1214 quantified through qPCR of the 16S rRNA gene using the Femto Bacterial DNA Quantification Kit 1215 (Zymo Research), which has a lower limit of detection of 20fg. Two sets of standards and negative 1216 controls were included in each qPCR run. The number of bacterial cells for each particle was 1217 estimated from the absolute DNA amounts based on measurements indicating a mean of $2.5 \mathrm{fg}$ 1218 DNA per bacterial cell in seawater samples (42).

1220 Metabolomics experiments and analyses. We performed untargeted metabolomics of the 1221 seawater that surrounded each chitin particle (after removing the chitin particles at each time point) 1222 and of the initial, unincubated seawater $(t=0)$. All samples were first diluted 1:100 in nuclease-free 1223 water (in two serial 1:10 dilutions). We used a binary LC pump (Agilent Technologies) and an MPS2 1224 Autosampler (Gerstel) coupled to an Agilent 6520 time-of-flight mass spectrometer (Agilent 1225 Technologies) operated in negative mode, at $2 \mathrm{GHz}$, extended dynamic range, with an $\mathrm{m} / \mathrm{z}$ 1226 (mass/charge) range of 50-1000. The mobile phase consisted of isopropanol:water $(60: 40, \mathrm{v} / \mathrm{v}) \mathrm{pH}$ 
12279 , with the addition of $5 \mathrm{mM}$ ammonium fluoride and a flow rate of $150 \mu \mathrm{l} / \mathrm{min}$. Raw data were 1228 processed and analyzed using preprocessing raw mass spectrometry data functions contained in 1229 the bioinformatics toolbox of $\operatorname{MATLAB}(43,44)$. We detected 5714 ions, of which 121 were 1230 annotated against a curated library of metabolites that are present in marine microbes, based on 1231 the BioCyc database (45). Certain ions were matched with multiple isomeric or isobaric compounds 1232 (as noted in Table S3). Detectable metabolites were those with ion intensities that passed the 1233 detection threshold above the inoculum [sample ion intensity $>$ (mean ion intensity at $\mathrm{t}=0)+$ $1234\left(3^{*}\right.$ standard deviation of ion intensity at $\left.\left.\mathrm{t}=0\right)\right]$. For metabolites that exceeded the limit of detection, 1235 the intensities of each ion were normalized between 0 and 1 , where 0 is the limit of detection and 12361 is the highest intensity measured of a given ion. Weighted ion intensities for each timepoint were 1237 calculated by taking the sum of all normalized intensities of ions in all samples for each timepoint. Sample collection and incubation with many chitin particles. Seawater was collected on the day of the experiment from Canoe Beach, Nahant, MA, USA (42 $\left.25^{\prime} 11.5^{\prime \prime} \mathrm{N}, 70^{\circ} 54^{\prime} 26.0^{\prime \prime} \mathrm{W}\right)$, the same source as seawater used elsewhere in this study. Chitin magnetic particles (New England 1242 Biolabs, \#E8036S) were collected on a $40 \mu \mathrm{m}$ cell strainer then passed through a $100 \mu \mathrm{m}$ cell 1243 strainer to restrict the size range of the particles to $40-100 \mu \mathrm{m}$ (Corning). The size selected particles 1244 were then resuspended in $0.2 \mu \mathrm{m}$-filtered natural seawater to create three suspensions: $807 \pm 99$ 1245 particles $/ \mathrm{mL}( \pm$ indicates standard deviation, $n=3$ ), $182 \pm 28$ particles $/ \mathrm{mL}(\mathrm{sd}, n=3)$, or $88 \pm 3$ 1246 particles $/ \mathrm{mL}$ (sd, $n=3$ ). Unfiltered natural seawater, containing microbes, was left undiluted, or 1247 diluted $1: 10$, or diluted 1:100 into $0.2 \mu \mathrm{m}$-filtered natural seawater to create three different initial 1248 densities of bacterioplankton. All combinations of particles and cells were combined by adding $5 \mathrm{~mL}$ 1249 particle mixture to $10 \mathrm{~mL}$ cell mixture to create a matrix of 9 separate conditions. Particle/cell 1250 mixtures were incubated in $15 \mathrm{~mL}$ polystyrene tubes (Falcon) with end-over end rotation at a rate 1251 of 8 revolutions/minute on a Stuart SB3 rotator at room temperature $\left(21-25^{\circ} \mathrm{C}\right)$.

1253 Imaging and quantification of chitin particle colonization by natural seawater bacteria. For 1254 the experiment incubating chitin particles individually in seawater (see the Methods section 
1255 "Seawater incubation with individual chitin particles"), at each time point, the communities on a 1256 subset of particles (that were not sequenced) were stained with the DNA stain SYTO9 (Invitrogen, 1257 \#S34854) at a final concentration of 500nM. STYO9 was added directly to the wells containing the 1258 particles and seawater, which were subsequently incubated in the dark at room temperature for 15 1259 minutes before the individual chitin particles were harvested (as described in the Method section 1260 "Seawater incubation with individual chitin particles") and mounted separately on microscope 1261 slides. Particles were imaged with a Zeiss epifluorescence microscope at 100X magnification. For 1262 the experiment incubating many particles together in seawater (see the Methods section "Sample 1263 collection and incubation with many chitin particles"), after 24 hours of incubation, $200 \mu$ samples 1264 of each condition were stained with SYTO9 at a final concentration of 5 $\mu$ M. The SYTO9-stained 1265 samples were transferred to a black-walled Greiner Bio-One $\mu$ Clear 96-well plate. Samples were 1266 imaged on an ImageXpress Micro Confocal (Molecular Devices) in widefield mode using a Nikon 1267 10x Plan Apo lambda objective (NA 0.45) and FITC filter (ex 482/35, em 536/40, dichroic $506 \mathrm{~nm}$ ) 1268 with blue LED illumination from a Lumencore Light Engine. Nine fields of view capturing the entire 1269 well were acquired to quantify all particles present in each well. For chitin particles incubated both 1270 individually and in bulk, a custom analysis script was written in MATLAB vR2019a (The Mathworks) 1271 to quantify the area of each chitin particle colonized by cells. The code defines chitin particle area, 1272 and the area of each particle covered by cells using intensity-based thresholds. Code and original 1273 data will be publicly available before publication at the following GitHub page: 1274 https://github.com/jaschwartzman/seawater_colonize 
a
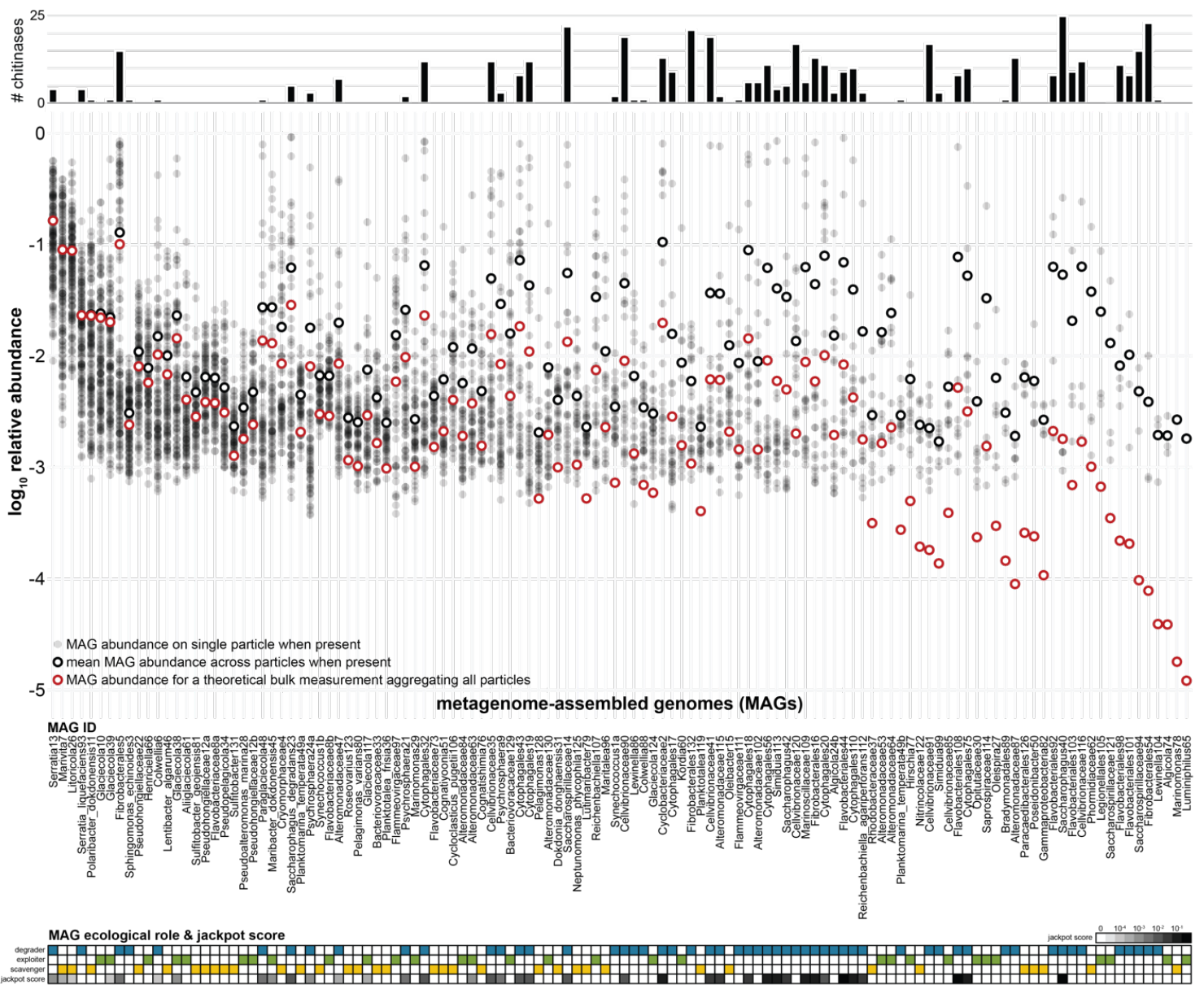

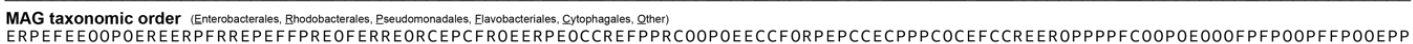

b

1276 Fig. S1. Taxonomic abundances averaged across particles do not represent the

1278 abundances of metagenome-assembled genomes (MAGs; $n=120$ ) across late-stage particles.

1279 Smaller black dots indicate the relative abundance of each MAG per particle $(n=149)$. Larger black 
1280 circles indicate the $\log _{10}[$ mean relative abundance] across the particles on which the MAG was

1281 found. Larger red circles indicate the $\log _{10}[$ mean relative abundance] across all the particles (i.e.

1282 the MAG abundances for a theoretical bulk measurement aggregating all particles). MAGs are

1283 sorted from left to right by their prevalence across particles (i.e. the number of particles on which

1284 they are detected). The bars above show the average number of chitinases encoded in each cluster

1285 of highly similar MAGs (see Methods). The annotations below show each MAG's taxonomic ID

1286 (matching Table S1); predicted ecological role (heatmap: blue = degrader, green = exploiter, yellow

1287 = scavenger); jackpot score (heatmap: white = low, black = high); and taxonomic order $(\mathrm{E}=$

1288 Enterobacterales, $\mathrm{R}=$ Rhodobacterales, $\mathrm{P}=$ Pseudomonadales, $\mathrm{F}=$ Flavobacteriales, $\mathrm{C}=$

1289 Cytophagales, $\mathrm{O}=$ Other). (b) Histogram of the percent variance explained in the abundance ranks

1290 of MAGs on each late-stage particle by the abundance ranks for a theoretical bulk measurement

1291 aggregating all particles (which is equivalent to the average abundance across all particles). If the

1292 MAG abundance rank of a single particle's community matched that of the theoretical average, the

1293 percent variance explained would be 100\% (right dashed line); however, the ensemble scale

1294 explained only an average of $11.7 \%$ (left dashed line) of the variance in abundance ranks at the

1295 single particle level. 


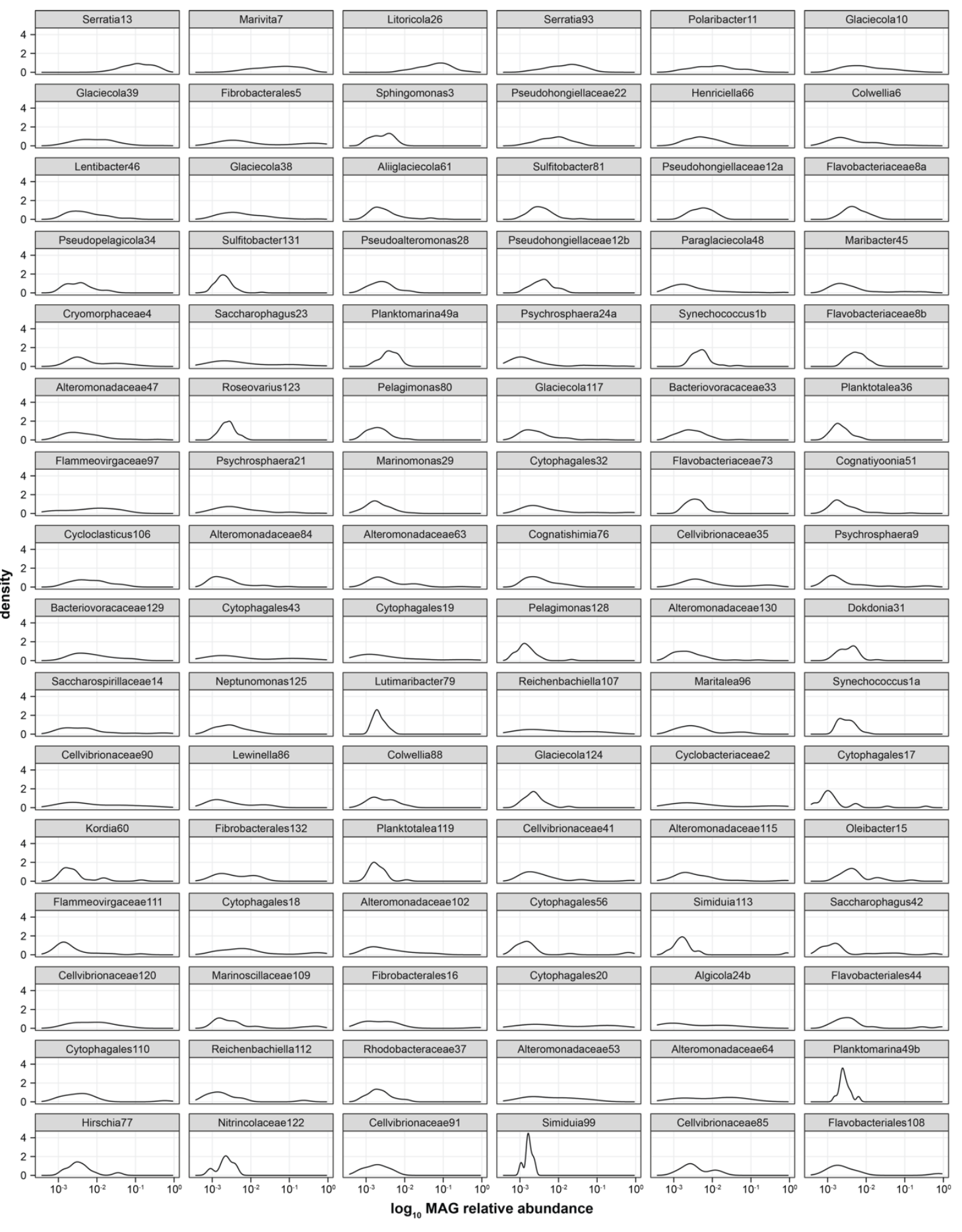

1297 Fig. S2. Distributions of MAG relative abundances on late-stage particles are approximately

1298 lognormal and right-skewed (i.e. towards high frequencies). Distributions are shown as

1299 Gaussian kernel density estimates for MAGs present on at least 10 late-stage particles. The area

1300 under each curve equals one. 
bioRxiv preprint doi: https://doi.org/10.1101/2021.09.27.461956; this version posted September 27, 2021. The copyright holder for this preprint (which was not certified by peer review) is the author/funder, who has granted bioRxiv a license to display the preprint in perpetuity. It is made available under aCC-BY-NC-ND 4.0 International license.

a

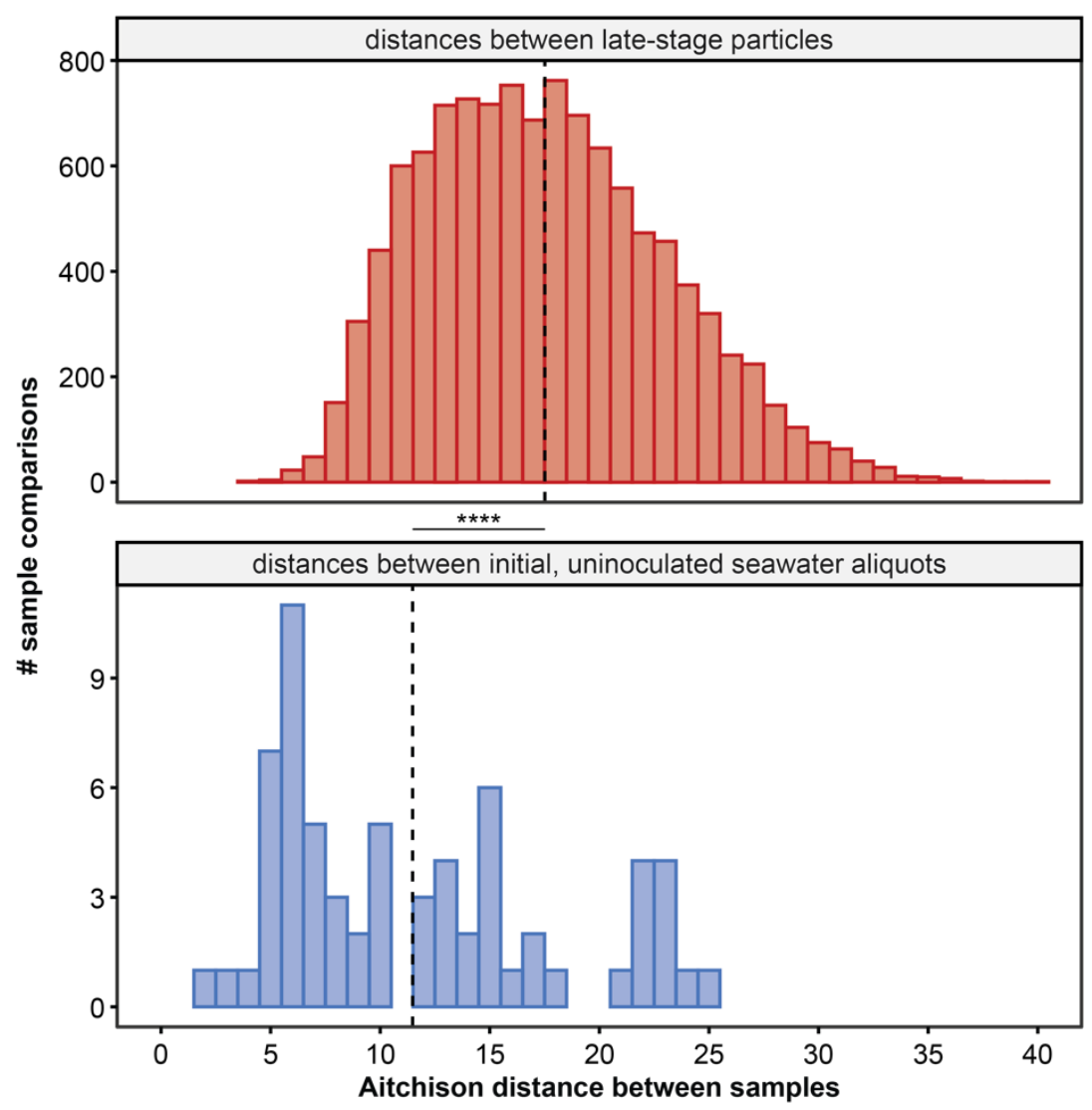

b

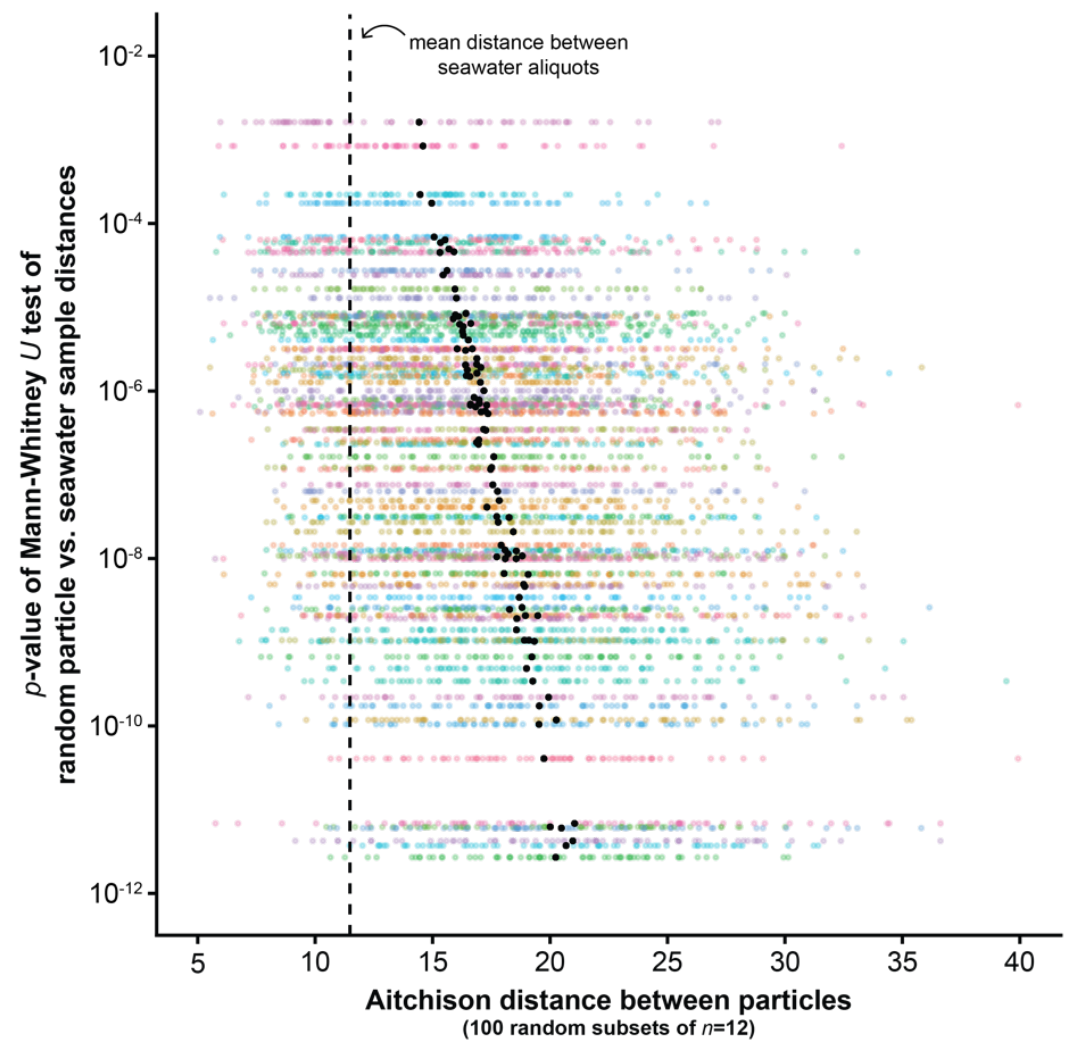


1302 Fig. S3. Taxonomic variability in the initial seawater does not significantly account for

1303 variability observed across late-stage particles. (a) Distributions of the Aitchison distances

1304 (Methods) calculated between all pairs of communities on late-stage particles $(n=149$, red

1305 histogram) and between all pairs of aliquots of unincubated, initial seawater $(n=12$, blue

1306 histogram). Dashed vertical lines represent the means of each distribution. Late-stage particles

1307 were significantly more dissimilar from one another than initial seawater samples (Mann-Whitney

$1308 U$ test: $p=1.3 \times 10^{-13}$ ). (b) The amount of inter-sample variability detected could depend on sample

1309 size, and many more pairs of particles than pairs of seawater samples were assessed in (a).

1310 Therefore, we calculated the Aitchison distances between random subsets of 12 late-stage

1311 particles and compared those distributions to that of the seawater samples. Small points represent

1312 inter-particle Aitchison distances calculated for 100 random subsets (each with its own point color),

1313 and black dots indicate the mean value for each subset. The inter-particle distances for each subset

1314 are plotted against the $p$-value from a Mann-Whitney $U$ test comparing the particle and seawater

1315 distributions. The dashed vertical line indicates the mean Aitchison distance between seawater

1316 samples (the same value as in the blue histogram in (a)). For all particle subsets, inter-particle

1317 distances were significantly higher than inter-seawater distances. 


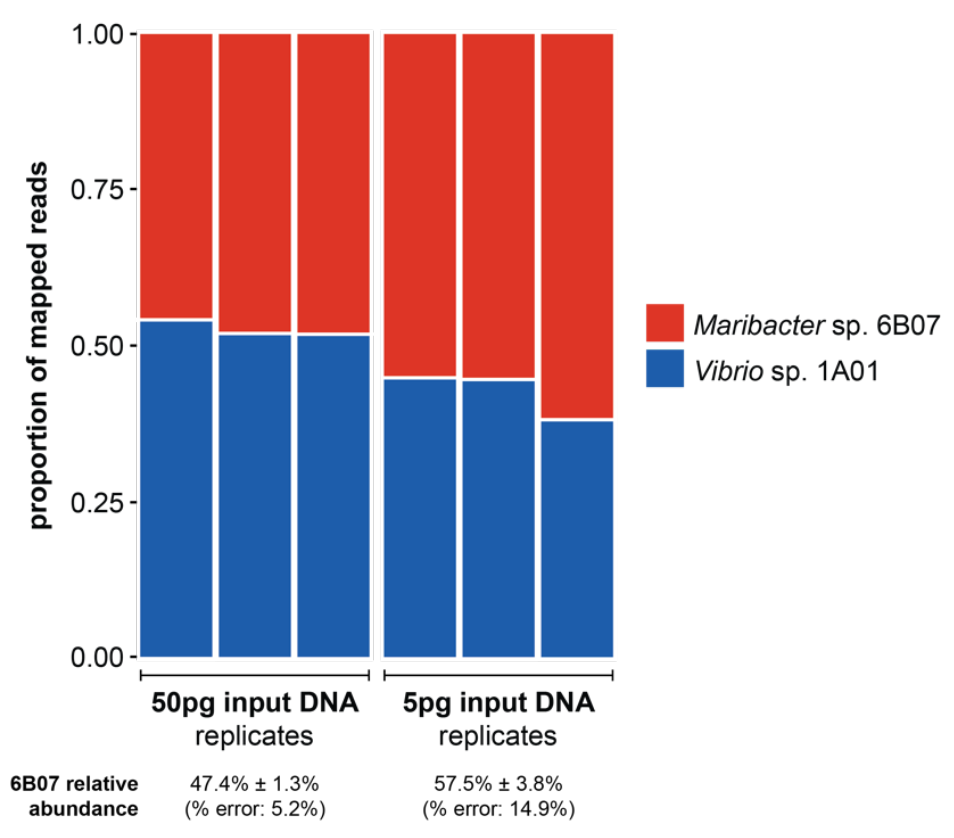

1319 Fig. S4. Mock communities sequenced with same protocols as particle-attached 1320 communities show relatively little deviation from expected strain abundances. See Methods

1321 for details on the preparation of the mock communities, which contained equal proportions of 1322 Marinobacter sp. 6B07 genomic DNA and Vibrio sp. 1A01 genomic DNA. Relative abundances 1323 estimated from metagenomic libraries prepared using $50 \mathrm{pg}$ of input DNA showed $5.2 \%$ error, 1324 whereas libraries prepared with 5 pg of input DNA showed $14.9 \%$ error. 
a
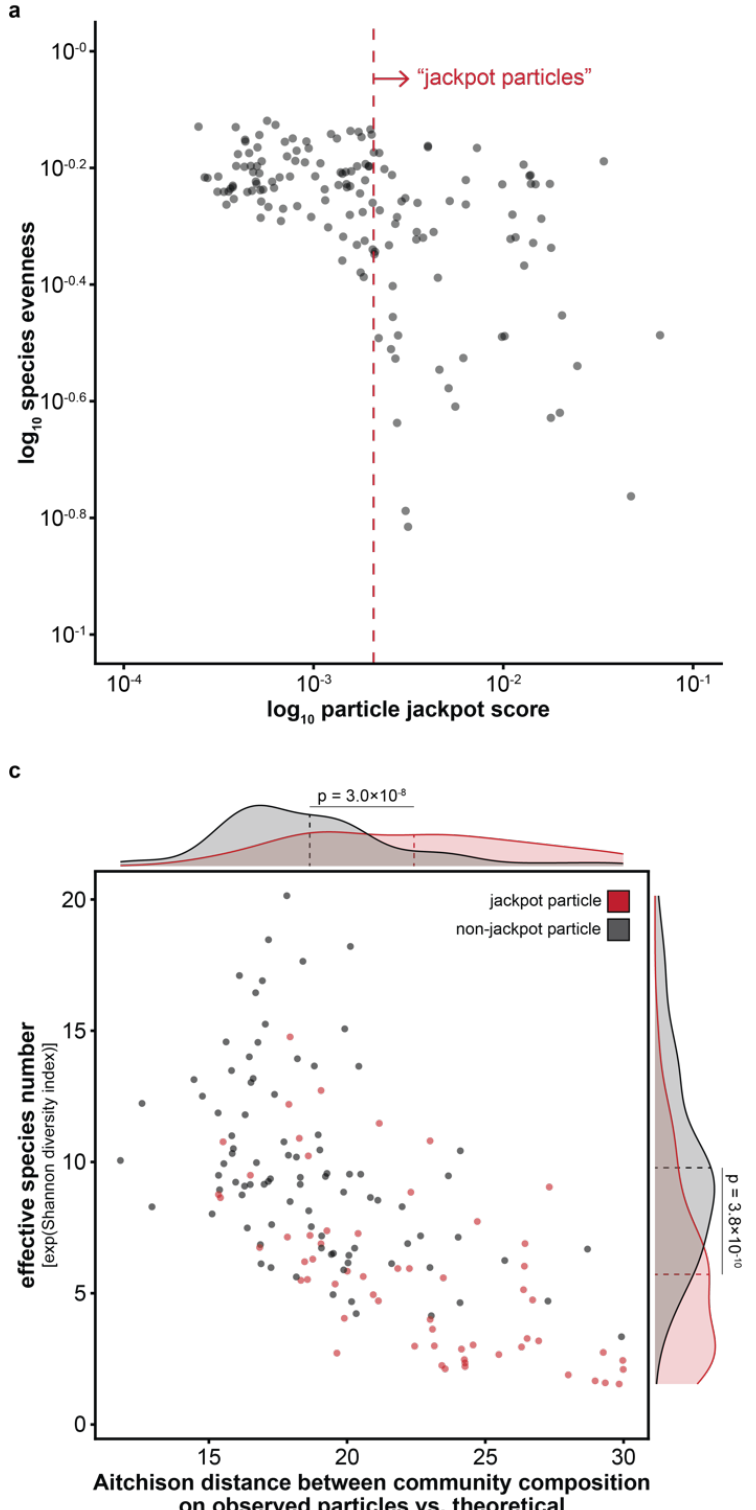
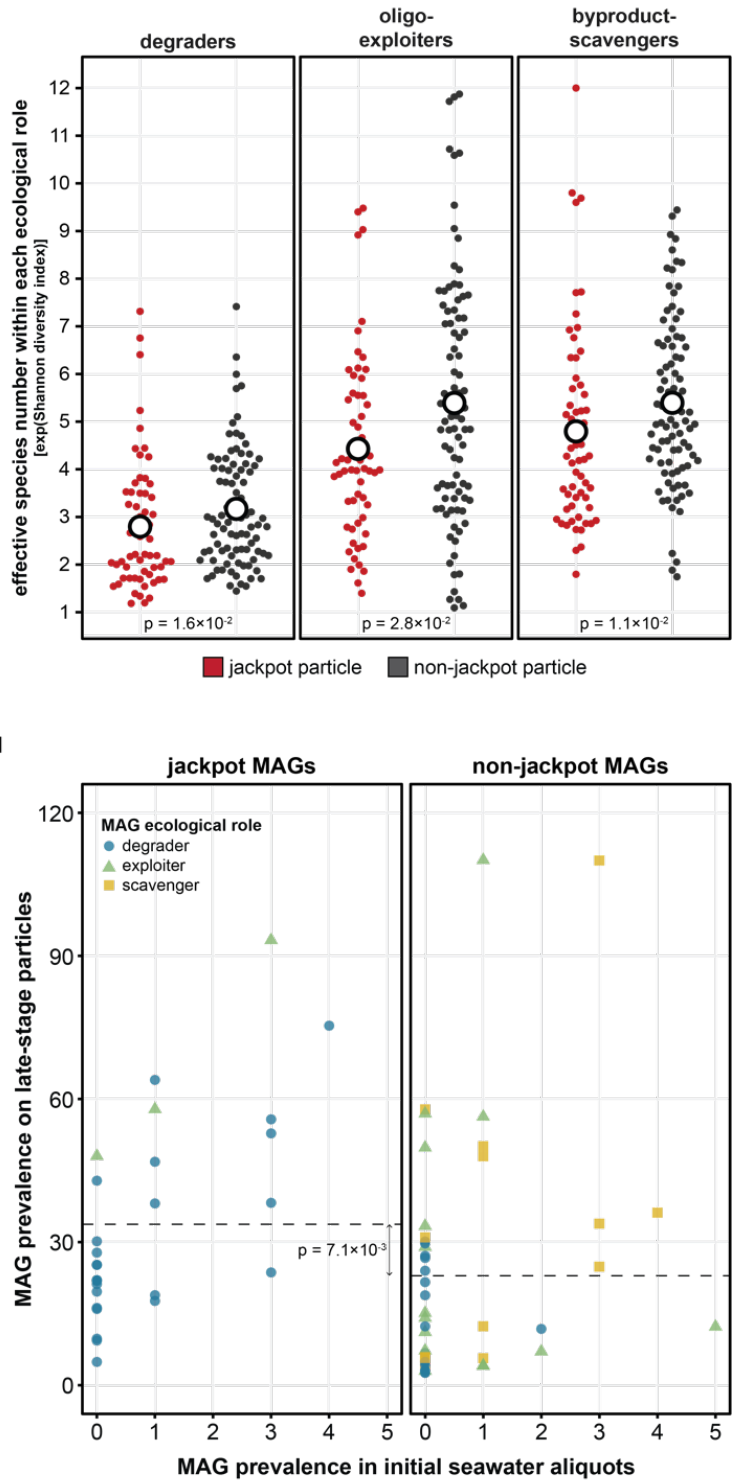

Fig. S5. Communities on jackpot particles are dominated by globally rare and locally

abundant strains. (a) Particles were defined as "jackpot particles" if they had high jackpot scores (indicating high relative abundances of jackpot taxa; see Methods for details). Each dot represents one late-stage particle $(n=149)$, and the red dashed line indicates the particle jackpot score threshold above which log-transformed values of Pielou's species evenness drop sharply. (b) The effective species numbers (calculated from the Shannon diversity index) within each ecological role. Each smaller dot represents a late-stage particle $(n=149)$, and dot color indicates whether the particle was a jackpot particle (red) or a non-jackpot particle (black). Larger white dots represent 
1334 the mean of each distribution. The diversity on jackpot particles was significantly lower than on non1335 jackpot particles for each of the roles (Mann-Whitney $U$ test: degraders $p=1.6 \times 10^{-2}$, exploiters $p=$ $13362.8 \times 10^{-2}$, scavengers $p=1.1 \times 10^{-2}$ ). (c) Community diversity (represented as effective species 1337 number, calculated from the Shannon diversity index) was inversely correlated (Spearman's $\rho=$ $\left.13380.68, p<2.2 \times 10^{-16}\right)$ with the Aitchison distance between the community composition observed on 1339 each particle and the composition of the theoretical average particle (see larger red circles in Fig. 1340 S1a). Each dot represents a late-stage particle $(n=149)$, and dot color indicates whether the 1341 particle was a jackpot particle (red) or a non-jackpot particle (black; see Methods for definitions). 1342 Jackpot particle communities were significantly less diverse (Mann-Whitney $U$ test: $p=3.8 \times 10^{-10}$ ) 1343 and more divergent from the theoretical average particle (Mann-Whitney $U$ test: $p=3.0 \times 10^{-8}$ ) than 1344 non-jackpot particles. (d) Jackpot taxa (left panel) were significantly more prevalent across late1345 stage particles (Mann-Whitney $U$ test: $p=7.1 \times 10^{-3}$ ) than non-jackpot taxa (right panel) that were 1346 equally rare across aliquots of the initial, unincubated seawater (prevalence in seawater samples, 1347 Mann-Whitney $U$ test: $p=0.33$; mean abundance in seawater samples, Mann-Whitney $U$ test: $p=$ 1348 0.49). Each point represents a MAG that was detected on fewer than half of the seawater aliquots, 1349 with the point color and shape indicating its predicted ecological role (blue circle $=$ degrader, green 1350 triangle $=$ exploiter, yellow square $=$ scavenger $)$. 

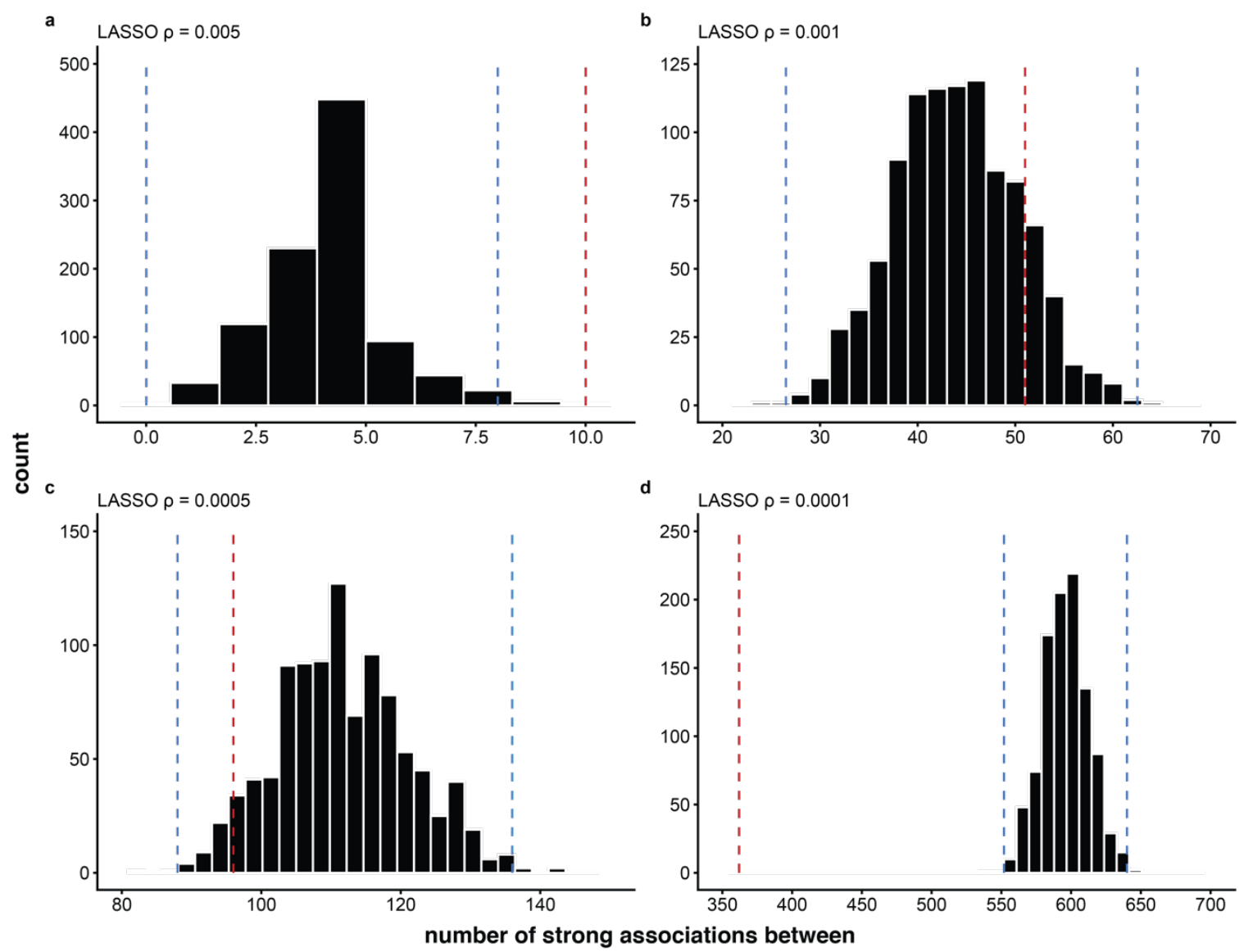
taxa for 1000 randomized datasets

Fig. S6. Late-stage particles exhibit little specific taxonomic structure. For the observed data, as well as for 1000 randomizations of the data, we inferred the number of conditional dependencies between taxa from the estimated inverse covariance matrix of center log-ratio-transformed relative abundances across late-stage particles. The inverse covariance matrices were calculated using a graphical lasso approach with a regularization parameter of (a) $\rho=0.005$, (b)

(b) $\rho=0.001$, (c) $\rho=$ 0.0005 , or (d) $\rho=0.0001$. Each plot shows the distribution of the number of conditional dependencies (with strengths $\geq 0.2$ or $\leq-0.2$ ) between MAGs inferred for the randomizations of the data. The red lines indicate the number of conditional dependencies (with strengths $\geq 0.2$ or $\leq-0.2$ ) inferred from the observed data. The blue lines indicate thresholds beyond which values are considered outliers relative to the distribution calculated for the randomized datasets (using the interquartile range $[I Q R]$ method - left lines indicate the value of $\left[Q_{1}-1.5 \times I Q R\right]$, and right lines indicate the value of $\left.\left[\mathrm{Q}_{3}+1.5 \times I \mathrm{QR}\right]\right)$. The choice of the regularization parameter in the analyses 
bioRxiv preprint doi: https://doi.org/10.1101/2021.09.27.461956; this version posted September 27, 2021. The copyright holder for this preprint (which was not certified by peer review) is the author/funder, who has granted bioRxiv a license to display the preprint in perpetuity. It is made available under aCC-BY-NC-ND 4.0 International license.

1364 used to estimate the number conditional dependencies between taxa resulted in more strong 1365 associations being inferred for the observed communities than the randomized ones only when a 1366 trivially small number of associations were inferred (panel a). Therefore, in terms of the number of 1367 strain-specific associations, the observed particles were either indistinguishable from, or less 1368 structured than, random communities. 
bioRxiv preprint doi: https://doi.org/10.1101/2021.09.27.461956; this version posted September 27, 2021. The copyright holder for this preprint (which was not certified by peer review) is the author/funder, who has granted bioRxiv a license to display the preprint in perpetuity. It is made available under aCC-BY-NC-ND 4.0 International license.

a

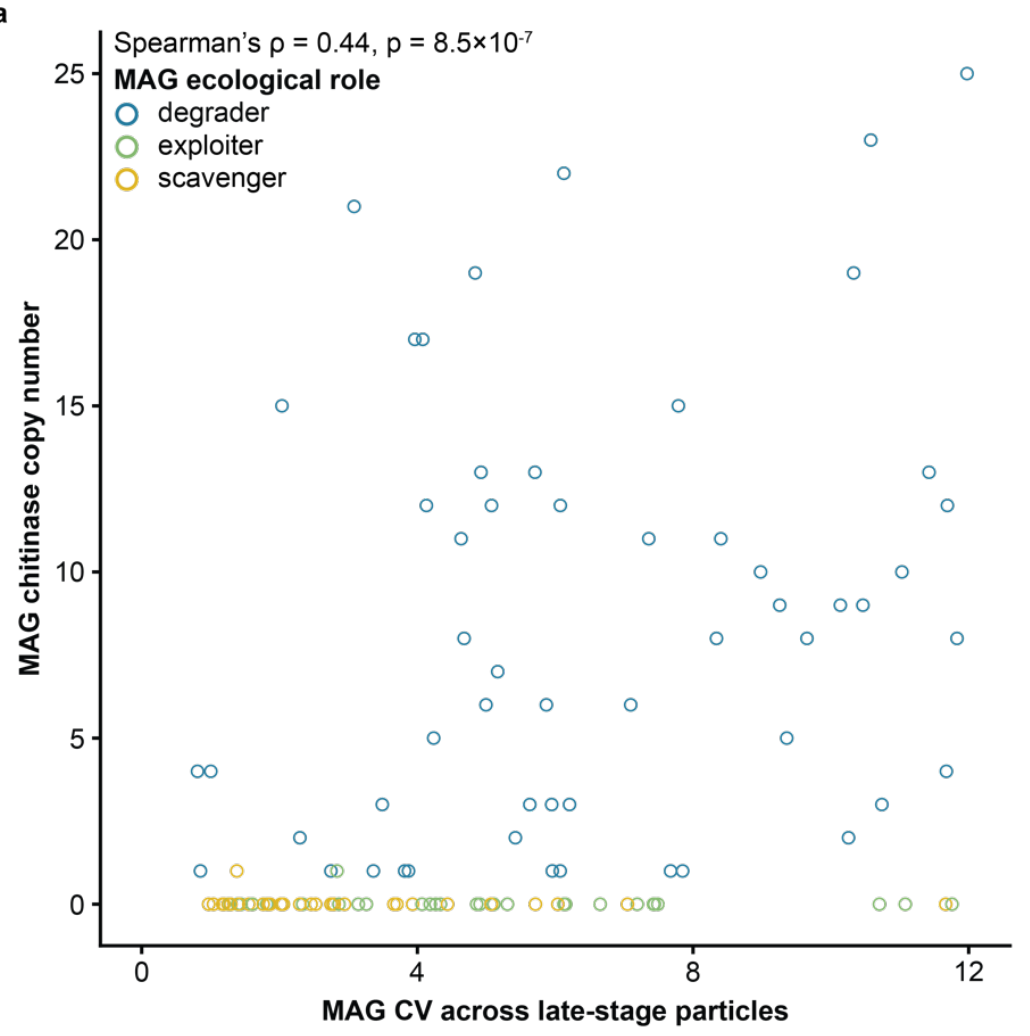

b

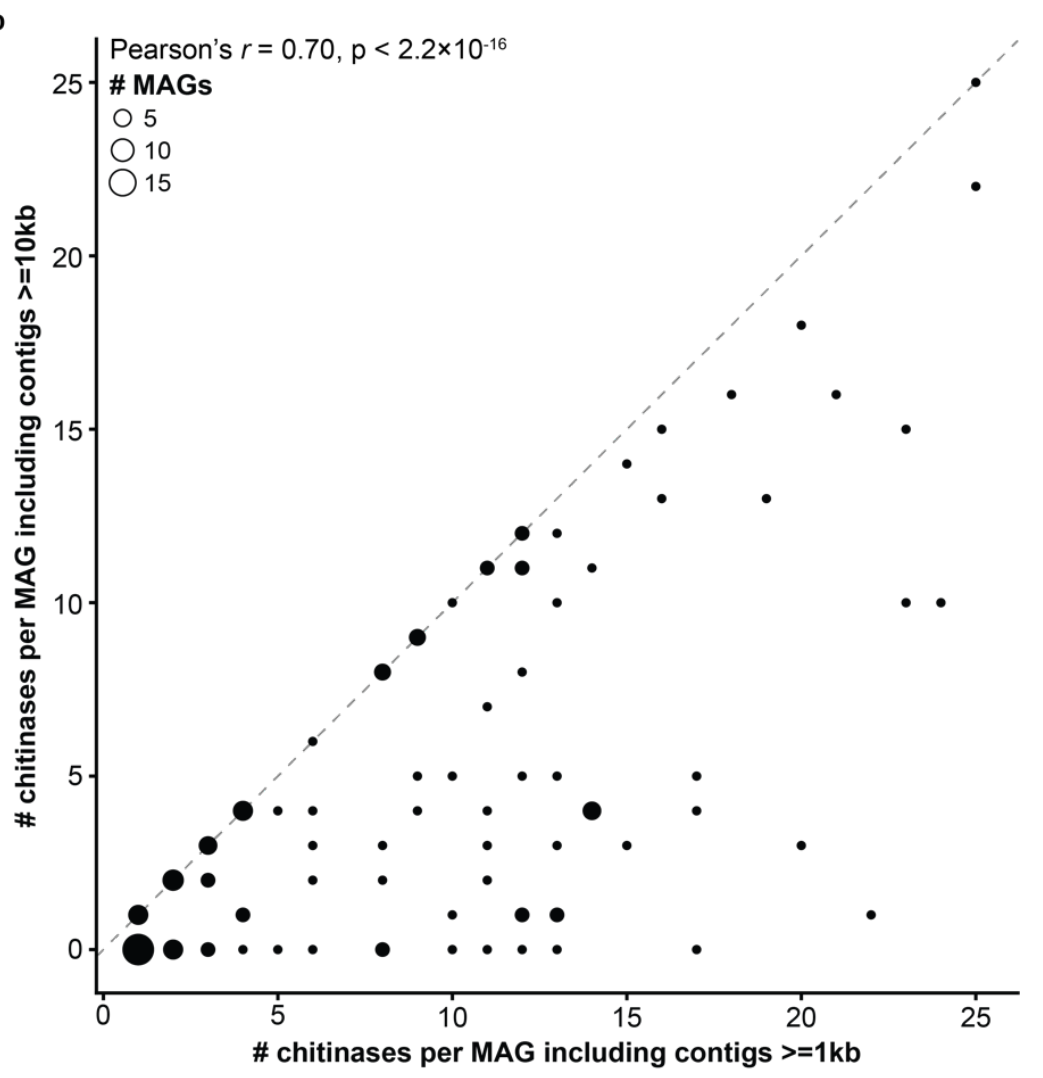


1370 Fig. S7. Highly variable taxa are often degrader strains encoding many chitinase genes. (a)

1371 There was a significant positive correlation between the coefficients of variation of MAG relative

1372 abundances across late-stage particles and the number of chitinase genes encoded in MAGs

1373 (Spearman's $\rho=0.44, p=8.5 \times 10^{-7}$; calculated for 120 MAGs across 149 particles). Each open dot

1374 represents a MAG, with the color indicating its predicted ecological role $($ blue $=$ degrader, green =

1375 exploiter, yellow = scavenger). (b) Comparison of the number of chitinase genes encoded by each

1376 MAG when considering contigs $\geq 10 \mathrm{~kb}$ (which are binned more reliably than shorter contigs) vs.

1377 considering contigs $\geq 1 \mathrm{~kb}$ (the minimum length of binned contigs). There was a strong correlation

1378 between chitinase copy numbers when considering contigs $\geq 10 \mathrm{~kb}$ vs. contigs $\geq 1 \mathrm{~kb}$ (Pearson's $r$

$1379=0.70, p<2.2 \times 10^{-16}$ ), lending confidence to estimates of high chitinase copy numbers in certain

1380 bins. Dot sizes indicate the number of MAGs at each coordinate. 


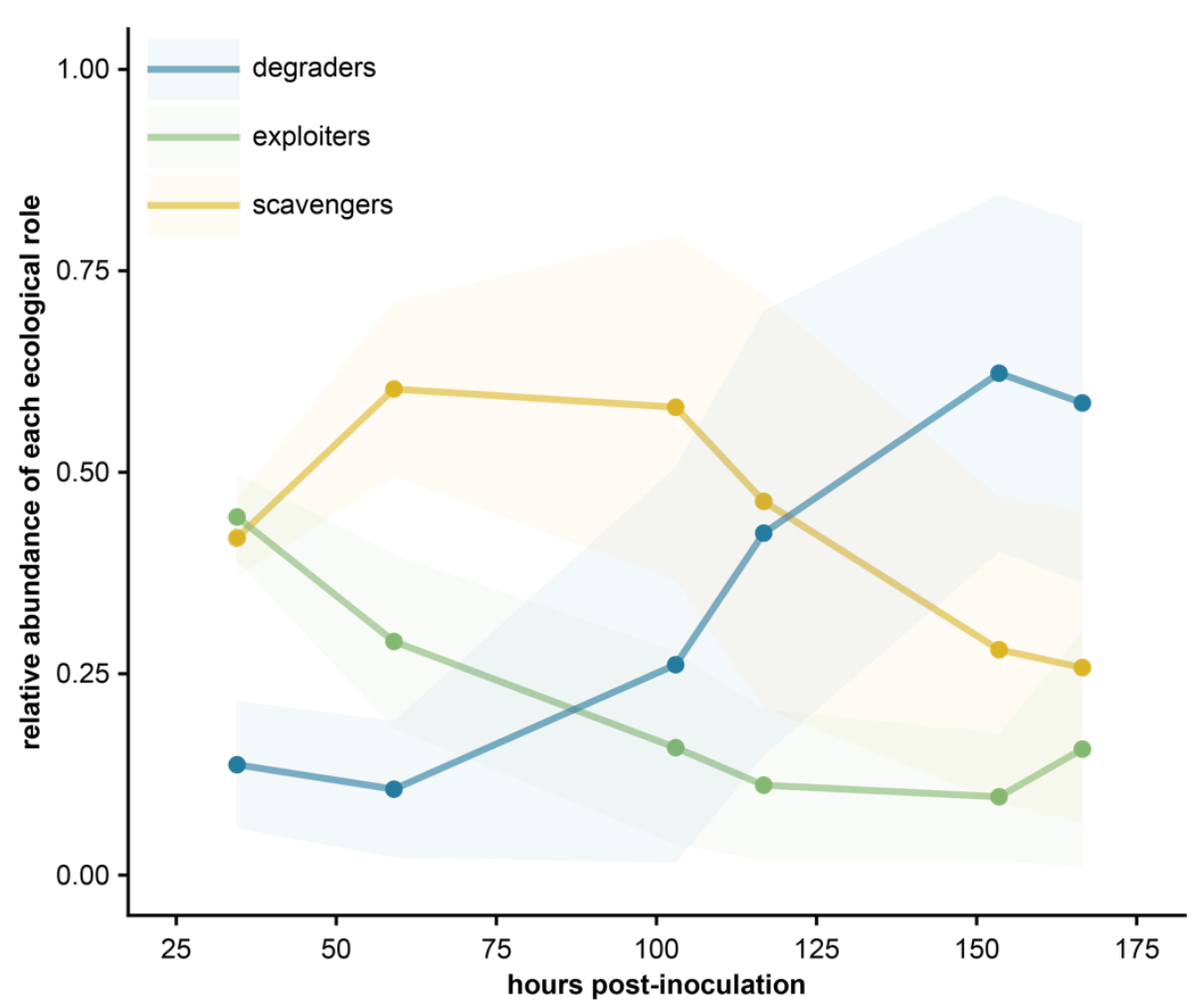

1382 Fig. S8. The proportion of predicted degraders on particles increases and becomes more

1383 variable over time. The relative abundances of the predicted ecological roles (degrader = blue,

1384 exploiter $=$ green, scavenger $=$ yellow) on particles harvested after varying incubation durations

1385 were calculated by summing the relative abundances of MAGs classified into each role. Points

1386 indicate mean values across particles at each time point, with shading representing \pm 1 standard

1387 deviation. The number of particles considered at each time point were (in order from early to late)

$1388 \quad 88,88,80,90,76$, and 73. 
1389

1390

1391
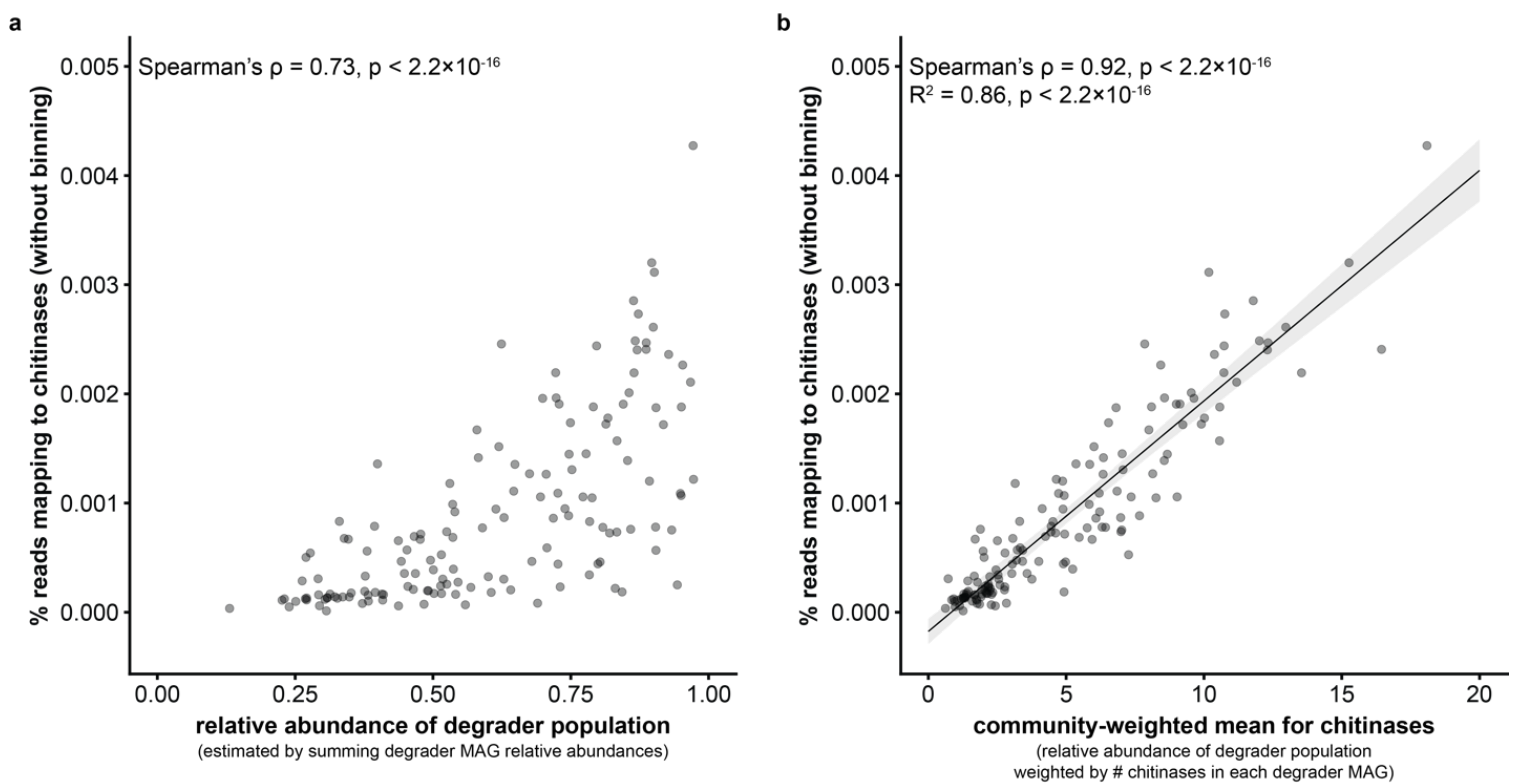

Fig. S9. The wide range of degrader population relative abundances estimated for late-stage particles holds when genes are used as read mapping references rather than MAGs.

Conceivably, the use of MAGs as reference genomes could have biased our estimate of the degrader population abundance; therefore, we also mapped reads to a reference set of chitinase genes (Methods). (a) There was a strong correlation (Spearman's $\rho=0.73, \mathrm{p}<2.2 \times 10^{-16}$ ) between the degrader population relative abundances estimated using MAGs and the percent of reads that mapped to chitinase genes. Each dot represents one late-stage particle $(n=149)$. (b) If the degrader population relative abundance estimated by MAGs were a consistent approximation of the true degrader population abundance, then the wide range in the number of chitinases encoded in each degrader MAG (Fig. S6, Table S1) would be reflected in the percent of reads in each community mapping to chitinase genes. Therefore, we weighted the degrader population relative abundances by the number of chitinases in each MAG to calculate the community-weighted mean for chitinases of each late-stage particle (Methods). There was an even stronger correlation (Spearman's $\rho=0.92, \mathrm{p}<2.2 \times 10^{-16}$ ) between the chitinase community-weighted mean estimated using MAGs and the percent of reads that mapped to chitinase genes. Each dot represents one late-stage particle $(n=149)$. The black line represents the linear regression line $\left(R^{2}=0.86, p<\right.$ $2.2 \times 10^{-16}$; shading indicates the $99 \%$ confidence interval). Therefore, reference MAGs captured a 
bioRxiv preprint doi: https://doi.org/10.1101/2021.09.27.461956; this version posted September 27, 2021. The copyright holder for this preprint (which was not certified by peer review) is the author/funder, who has granted bioRxiv a license to display the preprint in perpetuity. It is made available under aCC-BY-NC-ND 4.0 International license.

1407 representative subsample of the degraders within particle-attached communities, and predictions 1408 of chitinolytic potential were consistent between MAG- and gene-based approaches. 


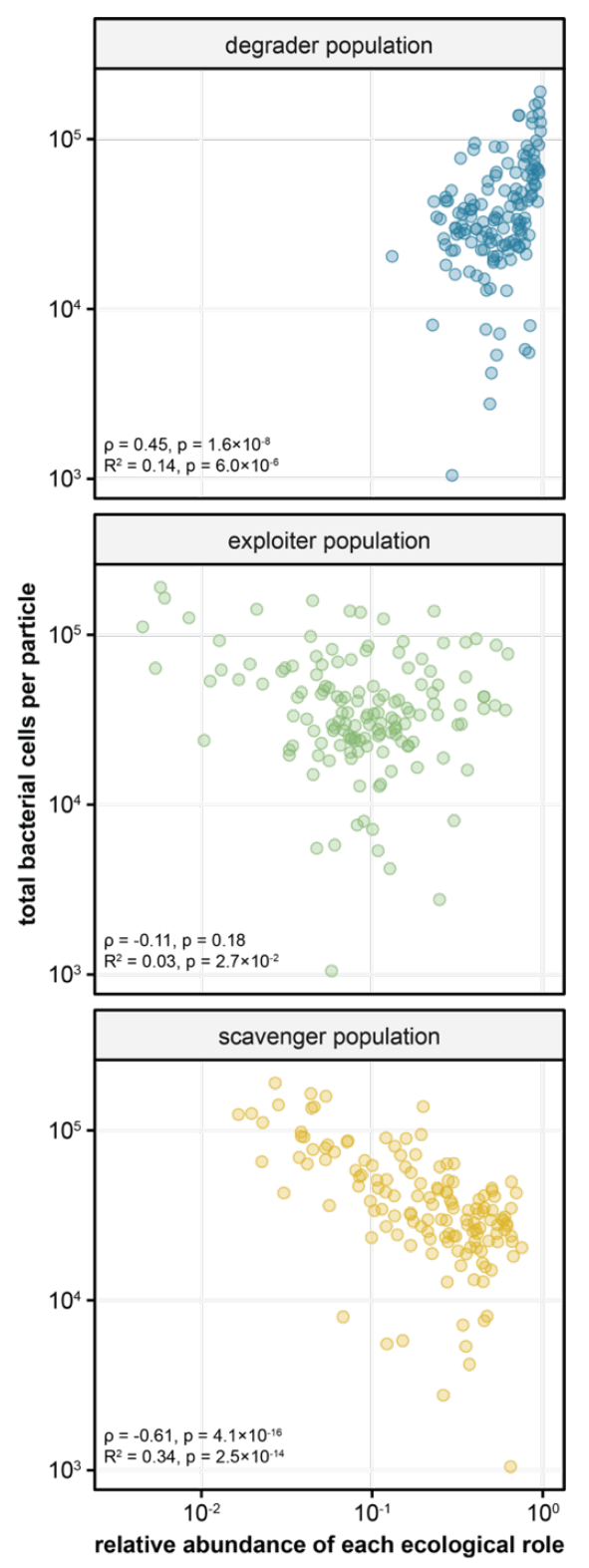

1410 Fig. S10: The overall yield of late-stage particles is correlated with community composition.

1411 There was a strong negative correlation between the proportion of scavengers and the number of

1412 bacterial cells in late-stage communities, estimated through qPCR (yellow dots, $n=142$;

1413 Spearman's $\rho=-0.61, p=4.1 \times 10^{-16}$; log-log linear regression: $\left.R^{2}=0.34, p=2.5 \times 10^{-14}\right)$. There was

1414 a less strong, though still highly significant, positive correlation between biomass and the proportion

1415 of degraders (blue dots; Spearman's $\rho=0.45, \mathrm{p}=1.6 \times 10^{-8}$; $\log$-log linear regression: $\mathrm{R}^{2}=0.14, \mathrm{p}$

$1416=6.0 \times 10^{-6}$ ), and there was no correlation with the exploiter population (green dots; Spearman's $\rho$

$1417=-0.11, p=0.18 ; \log -\log$ linear regression: $\left.R^{2}=0.03, p=2.7 \times 10^{-2}\right)$. 
a

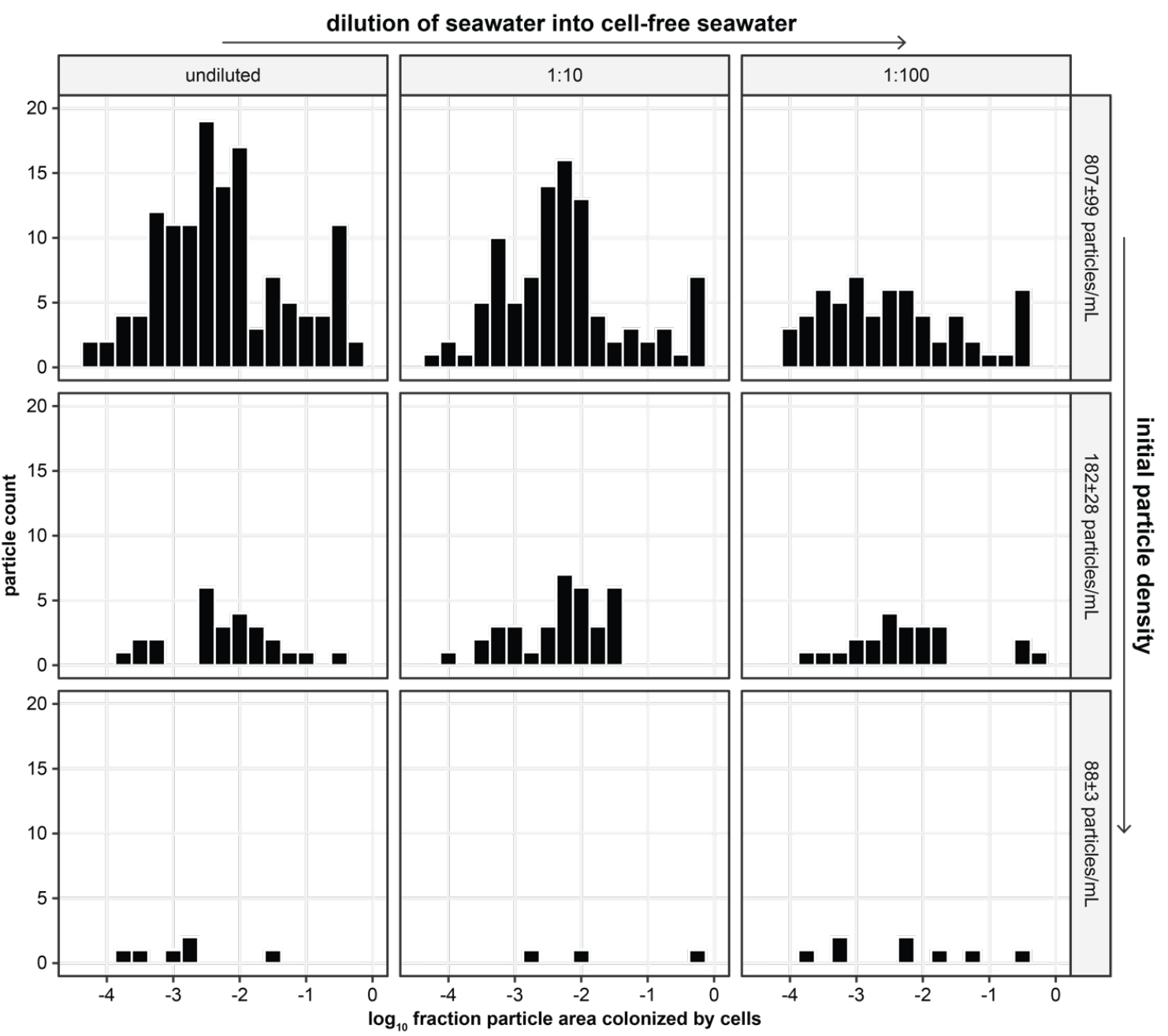

b

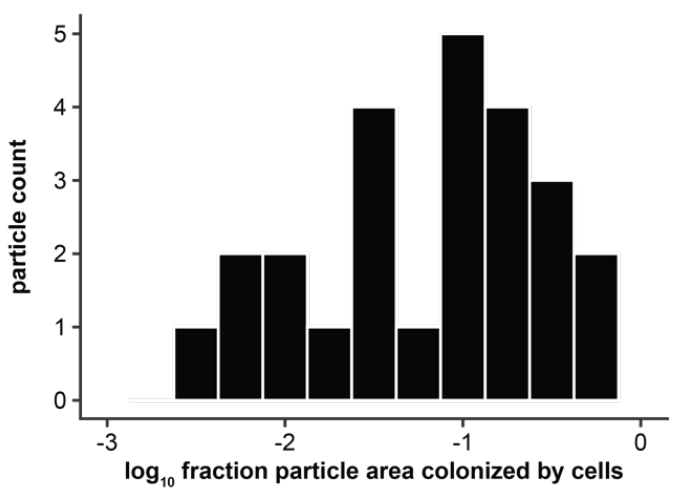

1419 Fig. S11. The cell counts on single particles incubated in the same volume of seawater span several orders of magnitude, matching the range estimated for single particles incubated separately. All plots show the distributions of the proportion of a particle's area occupied by cells 
1422 (transformed on a $\log _{10}$ scale), estimated by visualizing particles stained with the DNA intercalating

1423 dye SYTO 9. (a) Cell count distributions for communities on single particles incubated in seawater

1424 together for 24 hours at various initial particle concentrations (top row: $807 \pm 99$ particles/mL; middle

1425 row: $182 \pm 28$ particles $/ \mathrm{mL}$; bottom row: $88 \pm 3$ particles $/ \mathrm{mL} ;, \pm$ indicates 1 standard deviation for $n=$

14263 replicates throughout) and at various initial cell concentrations (left column: undiluted natural

1427 seawater; middle column: seawater inoculum diluted 1:10 into $0.2 \mu \mathrm{m}$-filtered natural seawater; right

1428 column: diluted 1:100). (b) Cell count distributions for communities on single particles incubated in

1429 seawater separately and harvested after 154-167 hours (i.e. late-stage communities). 


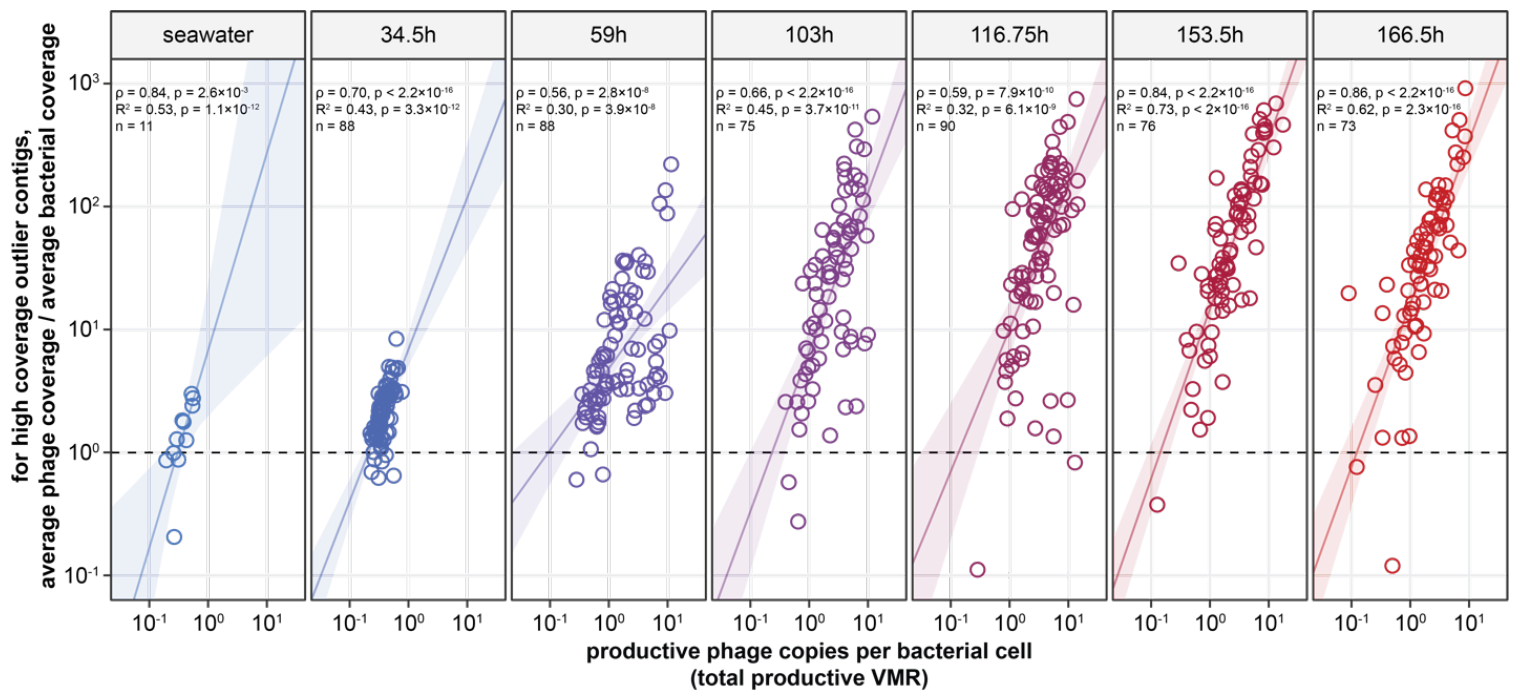

1431 Fig. S12. Phage-derived contigs that are coverage outliers have much higher average read

1432 coverage than bacteria-derived contigs that are coverage outliers. Given that read coverage

1433 values from metagenomic data are often noisy, it is conceivable that productive phage contigs had

1434 unusually high coverage simply due to sequencing noise. However, for contigs that were high

1435 coverage outliers, the ratio for each particle (open dots) of the average coverage of phage contigs

1436 to the average coverage of bacterial contigs was often much greater than 1 (the black horizontal

1437 dashed line). Notably, these coverage ratios were overall lowest in the initial seawater inocula and

1438 rose during the incubation period, coinciding with the timescale of increasing mean productive

1439 VMRs (Fig. 4c). This indicates that phage contigs with high coverage values represented phages

1440 that were replicating more than their bacterial hosts, rather than representing contigs with randomly

1441 higher coverage values. Furthermore, there were strong positive relationships between this

1442 coverage ratio and the total productive VMR at each time point (see each subplot for significance

1443 values; the solid lines represent the log-log linear regression lines, and shading indicates the 95\%

1444 confidence intervals). Thus, as expected, the particles on which high outlier phage contig coverage

1445 was indistinguishable from high outlier bacterial contig coverage were mostly those with low

1446 productive VMRs. 

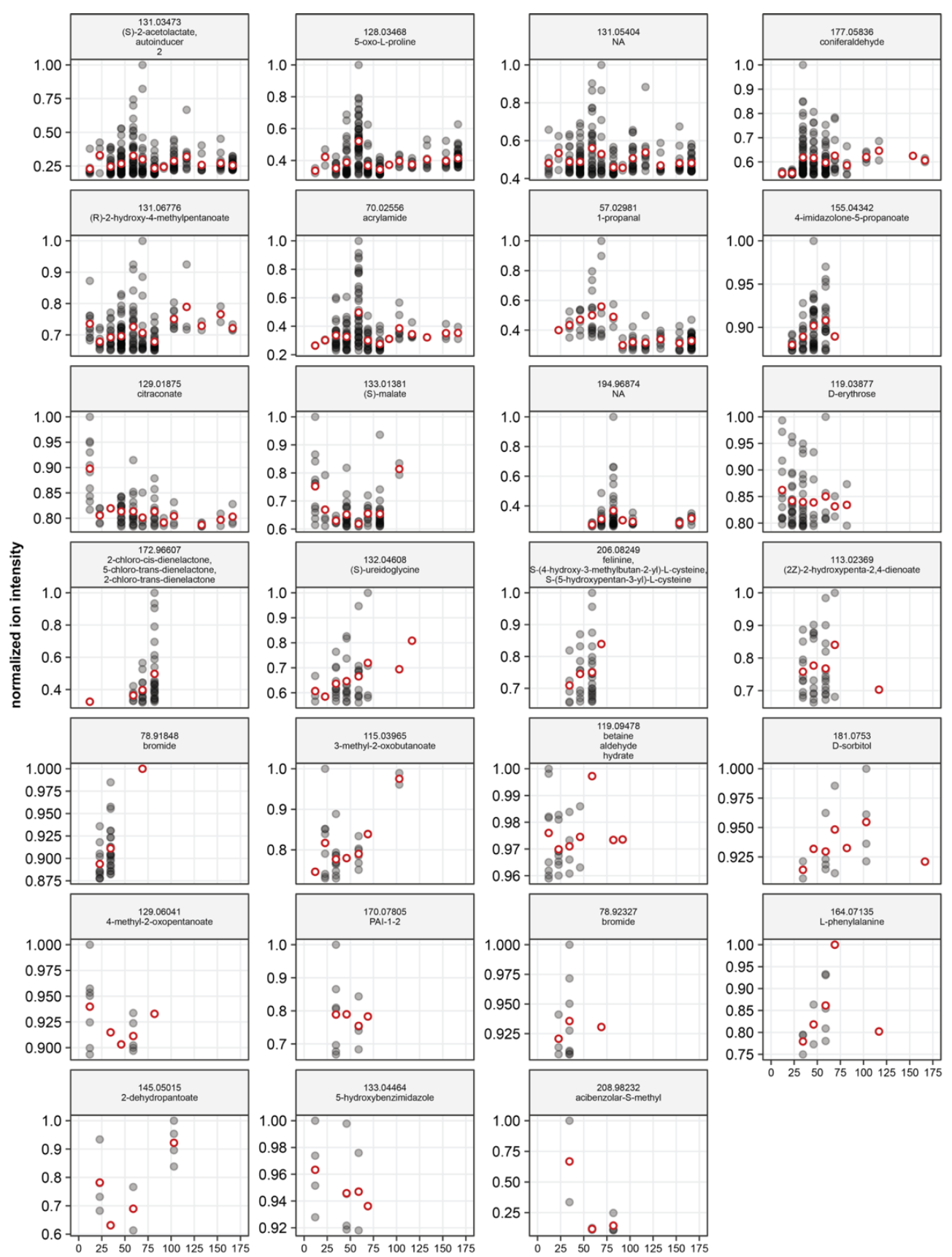

hours post inoculation

1448 Fig. S13. Normalized intensities of individual ions over time. The normalized intensities of ions

1449 across particles harvested after varying incubation durations are shown for ions that were significantly enriched (relative to the initial seawater) on at least 10 particles (see Methods). Gray dots indicate measurements for individual particles, and red circles represent the mean normalized intensities at each time point. Panel labels include the $\mathrm{m} / \mathrm{z}$ ratio and predicted annotation for each ion (see Methods). 
a
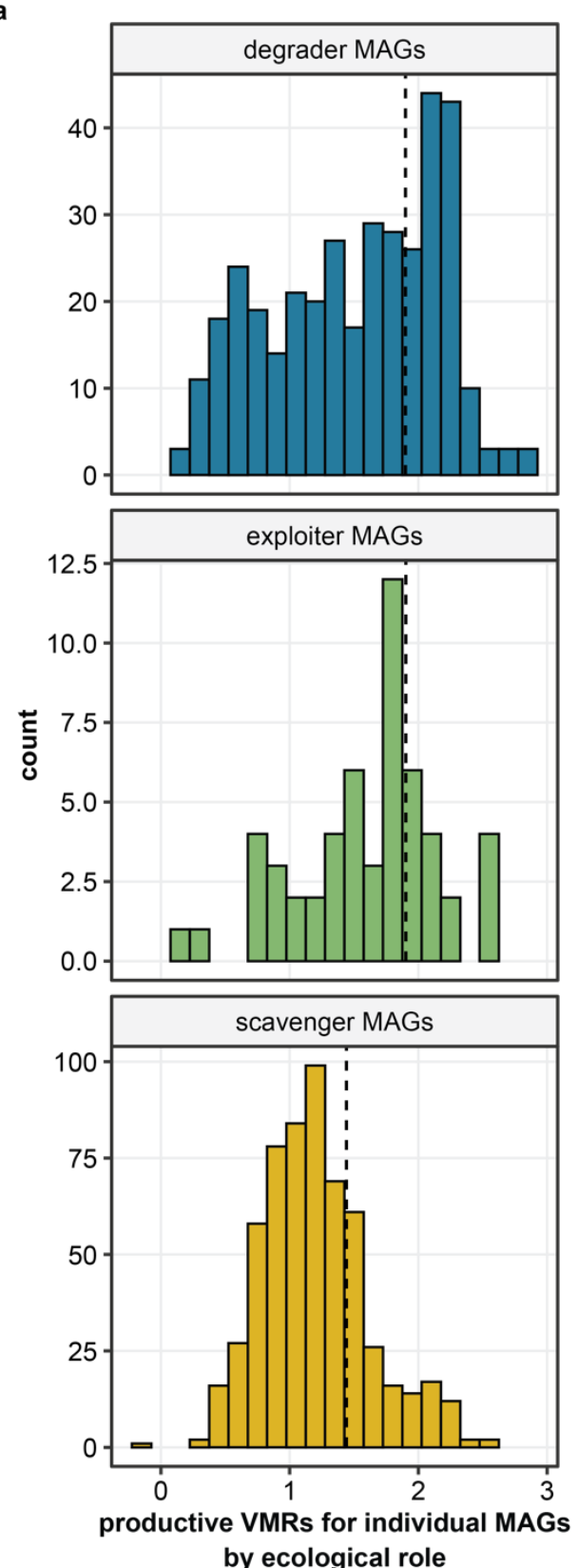

b
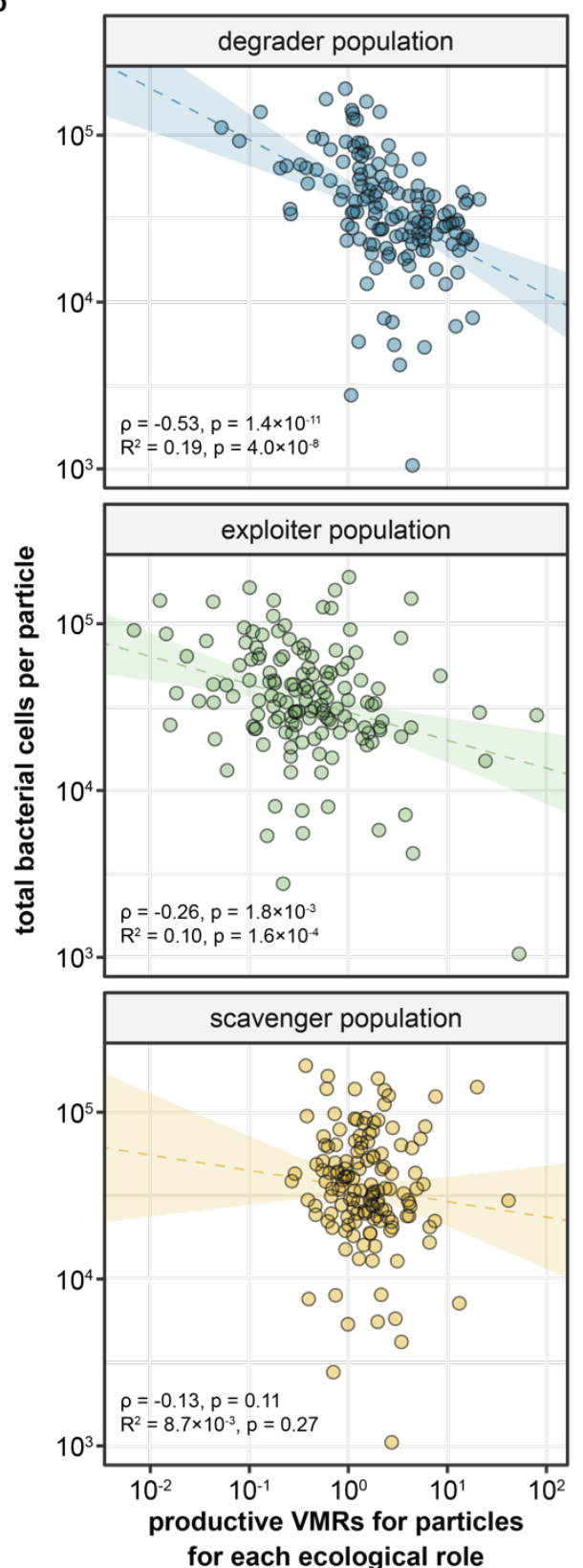

1455 Fig. S14. The degrader population contributes significantly to particle-level productive

1456 VMRs. (a) Degrader MAGs (top panel, blue distribution) and exploiter MAGs (middle panel, green

1457 distribution) had significantly higher productive VMRs across late-stage particles than scavenger

1458 MAGs (bottom panel, yellow distribution) when phages were productive. Distributions depict the

1459 non-zero VMRs for each group of MAGs (dashed lines represent the means of each distribution,

1460 with degraders having a mean VMR of 76.8, exploiters 78.6, and scavengers 27.3; one-way 
1461 ANOVA: $F(998,2)=47.4, p=2.1 \times 10^{-20}$; Tukey's HSD test: degrader-exploiter $p=0.40$; degrader1462 scavenger $p<1.0 \times 10^{-7}$; exploiter-scavenger $\left.p=1.0 \times 10^{-7}\right)$. When instances of VMRs equaling zero 1463 are included in the distributions, degraders had a mean VMR of 5.9, exploiters 1.3, and scavengers 14642.6 (one-way ANOVA: $F(14049,2)=42.0, p=6.3 \times 10^{-19}$; Tukey's HSD test: degrader-exploiter $p<$ $14651.0 \times 10^{-7}$; degrader-scavenger $p<1.0 \times 10^{-7}$; exploiter-scavenger $p=3.5 \times 10^{-2}$ ). This suggests that 1466 degraders overall experienced the most phage activation. (b) Absolute bacterial cell counts on late1467 stage particles $(n=142)$, estimated through qPCR, vs. each particle's productive VMR for the 1468 MAGs in each ecological role. Cell counts were negatively correlated with productive VMRs most 1469 strongly and significantly for degraders (top panel, blue dots; Spearman's $\rho=-0.53, p=1.4 \times 10^{-11}$ ) 1470 and less so for exploiters (middle panel, green dots; Spearman's $\rho=-0.26, p=1.8 \times 10^{-3}$ ), and there 1471 was no correlation between cell counts and productive VMRs for scavengers (bottom panel, yellow 1472 dots; Spearman's $\rho=-0.13, p=0.11$ ). Dashed lines represent the log-log linear regression lines 1473 between cell counts and productive VMR (degraders: $R^{2}=0.19, p=4.0 \times 10^{-8}$; exploiters: $R^{2}=0.10$, $1474 p=1.6 \times 10^{-4}$; scavengers: $R^{2}=8.7 \times 10^{-3}, p=0.27$; shading indicates the $95 \%$ confidence intervals). 1475 The productive VMRs for each ecological role were also significantly different from each other, with 1476 degraders having the highest mean VMR (one-way ANOVA: $F(423,2)=96.6, p=2.9 \times 10^{-35}$; Tukey's

1477 HSD test: degrader-exploiter $p<1.0 \times 10^{-7}$; degrader-scavenger $p=1.7 \times 10^{-3}$; exploiter-scavenger $\left.1478 p=<1.0 \times 10^{-7}\right)$. This suggests that the effect of phage activation on particle yield was largely driven 1479 by the degrader trophic level. 
a

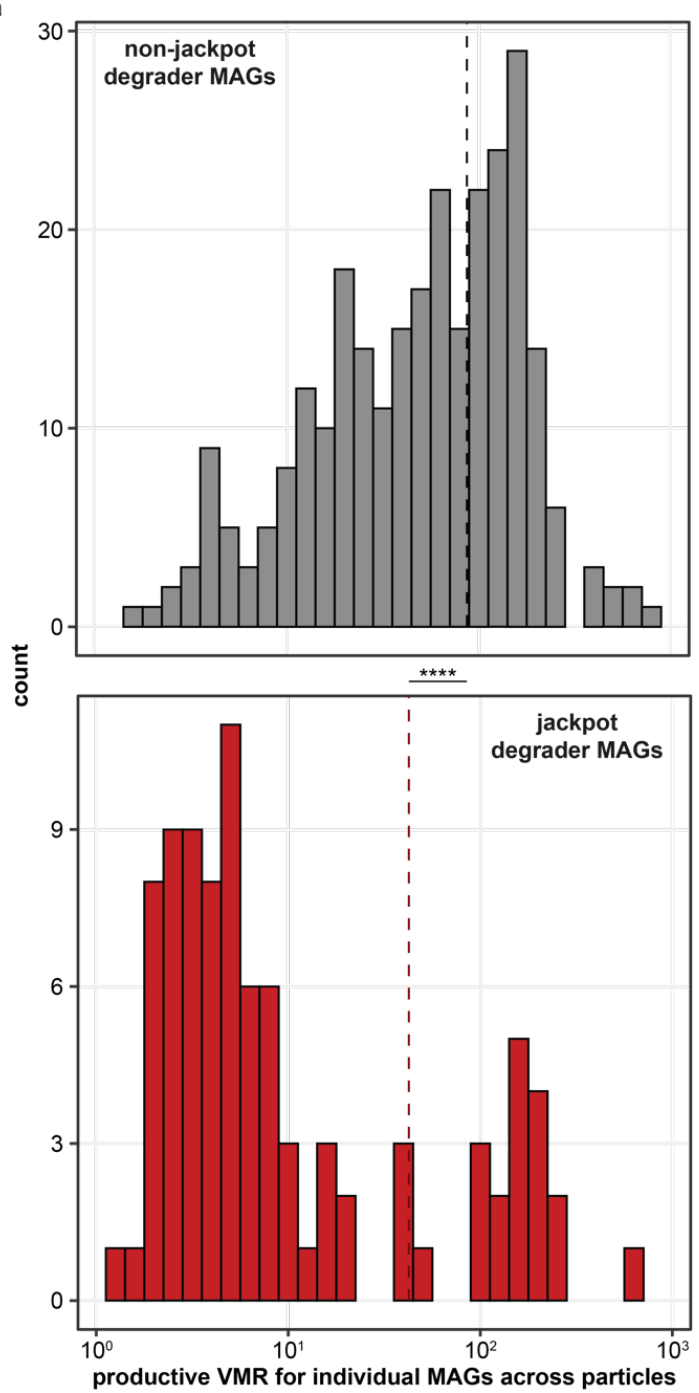

b

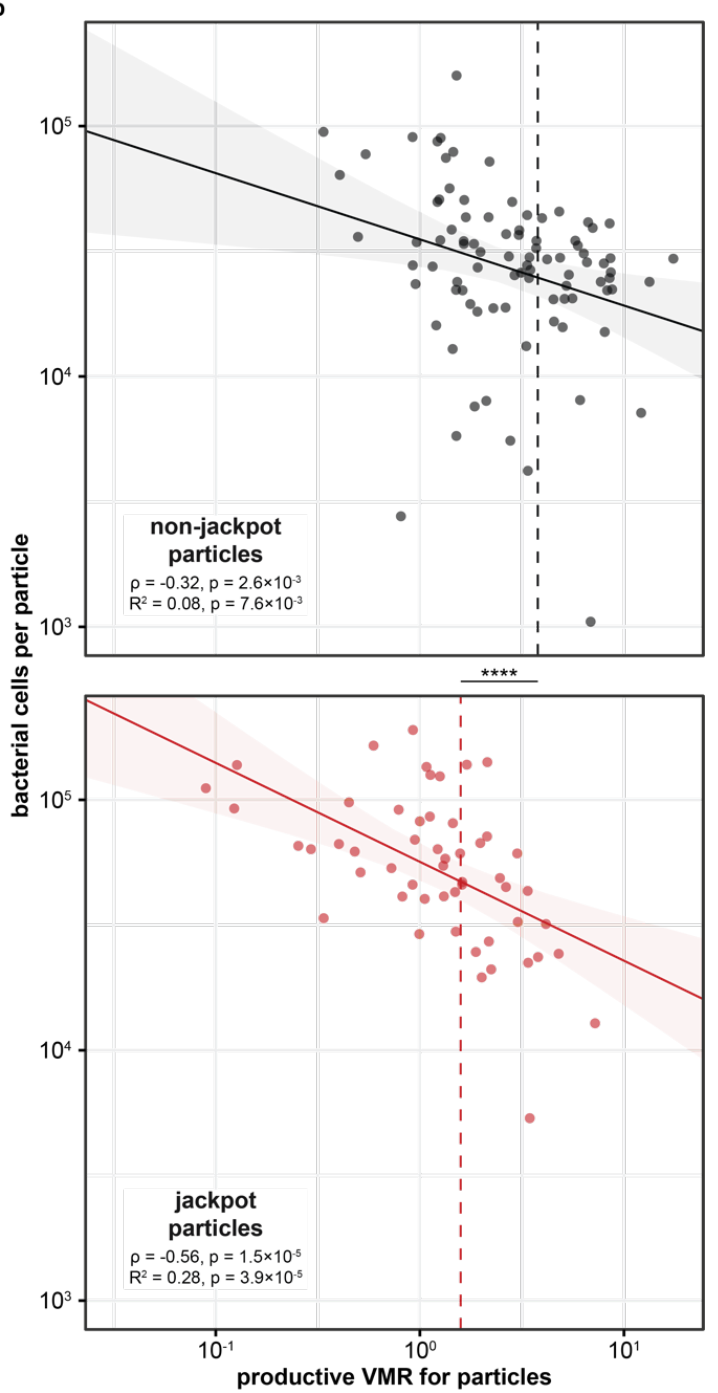

Fig. S15. The jackpot growth phenomenon is associated with less phage activation. (a)

1482 Jackpot degrader MAGs (bottom panel, red distribution) had lower productive VMRs across latestage particles than non-jackpot degraders (top panel, grey distribution). Distributions depict the non-zero VMRs for each group of MAGs (dashed lines represent the means of each distribution, with jackpot degraders having a mean VMR of 42.4 and non-jackpot degraders having a mean of 88.0; Mann-Whitney $U$ test: $\left.p=6.2 \times 10^{-15}\right)$. When instances of VMRs equaling zero are included in the distributions, jackpot degraders still have a lower mean VMR (1.32 vs. 12.9; Mann-Whitney $U$ test: $\left.p=1.3 \times 10^{-49}\right)$. (b) Jackpot particles had lower productive VMRs than non-jackpot particles.

1489 Modified version of Fig. 4e in which jackpot particles (bottom panel, red dots) are shown separately 1490 from non-jackpot particles (top panel, dark gray dots). Jackpot particles had significantly lower 
1491 productive VMRs than non-jackpot particles (dashed lines represent the means of each distribution;

1492 Mann-Whitney $U$ test: $p=4.3 \times 10^{-8}$ ), even controlling for differences in biomass between these

1493 groups of particles (ANCOVA: $F(1,139)=16.92, p=4.1 \times 10^{-4}$, partial $\eta^{2}=0.09$ ). Both groups of

1494 particles showed significant negative relationships between biomass (estimated through qPCR)

1495 and productive VMR (jackpot particles: Spearman's $\rho=-0.56, p=1.5 \times 10^{-5}$; non-jackpot particles:

1496 Spearman's $\rho=-0.32, p=2.6 \times 10^{-3}$ ). The solid lines represent the log-log linear regression lines

1497 between cell counts and productive VMRs (red line for jackpot particles: $R^{2}=0.28, p=3.9 \times 10^{-5}$;

1498 black line for non-jackpot particles: $R^{2}=0.08, p=7.6 \times 10^{-3}$; shading indicates the $95 \%$ confidence 1499 intervals). 

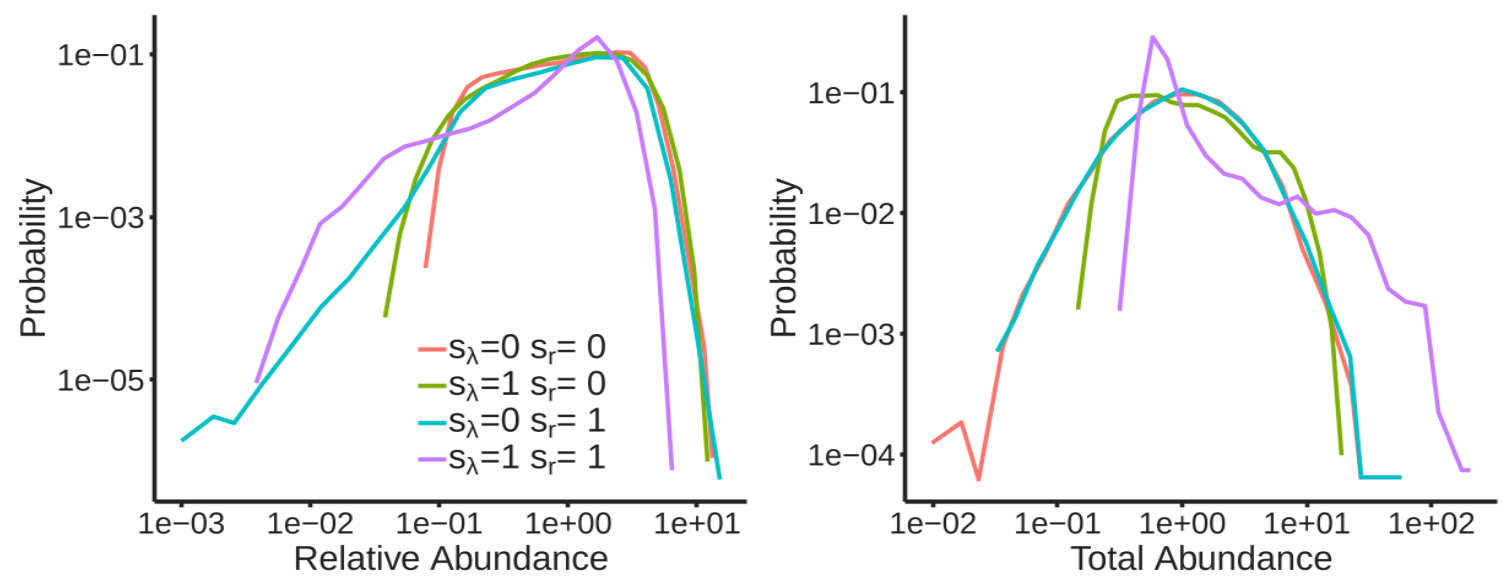

1501 Fig. S16. Numerical simulations of mathematical model \#1. The left panel shows the distribution

1502 of rescaled relative abundances averaged over MAGs. For each MAG the logarithm of the relative

1503 abundances across particles was rescaled by mean and variance, so that it had mean zero and

1504 unit variance. Lines represent averages over MAGs. Colors refer to a particular parameterization.

1505 The right plot shows the distribution of the total biomass across particles. In all the simulations we

1506 set $\bar{\lambda}=1$ and $\bar{r}=1$ (where the total time of the experiment was also set to be equal to 1 ). Different

1507 values of $s_{r}$ and $s_{\lambda}$ correspond to different colors. 

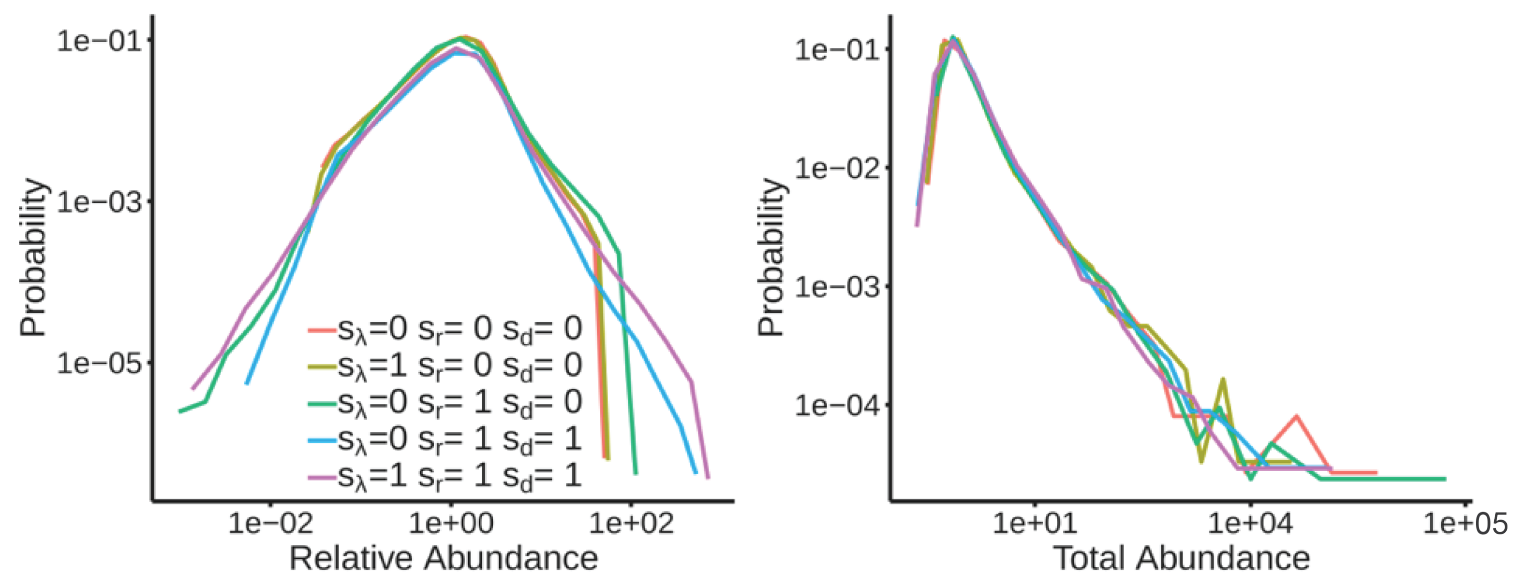

1509 Fig. S17. Numerical simulations of mathematical model \#2. The left panel shows the distribution

1510 of rescaled relative abundances averaged over MAGs. For each MAG the logarithm of the relative

1511 abundances across particles was rescaled by mean and variance, so that it had mean zero and

1512 unit variance. Lines represent averages over MAGs. Colors refer to a particular parameterization.

1513 The right plot shows the distribution of the total biomass across particles. In all the simulations we

1514 set $\bar{\lambda}=1$ and $\bar{r}=1$ (where the total time of the experiment was also set to be equal to 1 ). Different

1515 values of $s_{r}$ and $s_{\lambda}$ correspond to different colors. 

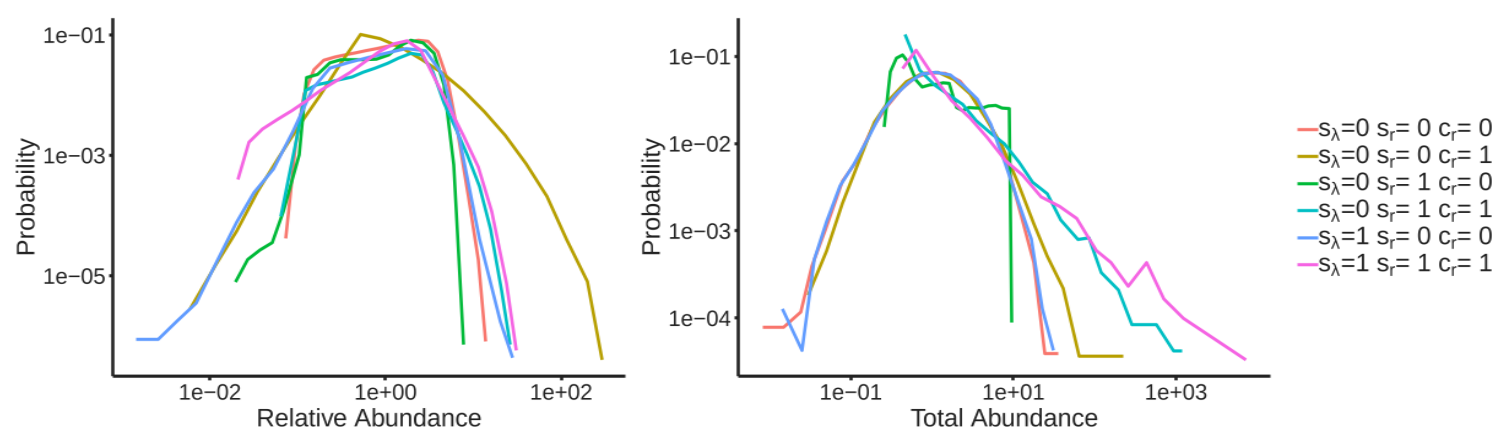

1517 Fig. S18. Numerical simulations of mathematical model \#3. Numerical simulations of model \#3.

1518 The panels show the same distributions as in Fig. S16. In all the simulations we set $\bar{\lambda}=1$ and $\bar{r}=1$

1519 (where the total time of the experiment was also set to be equal to 1). Different values of $s_{r}, s_{\lambda}$ and

$1520 \quad c_{r}$ correspond to different colors. 

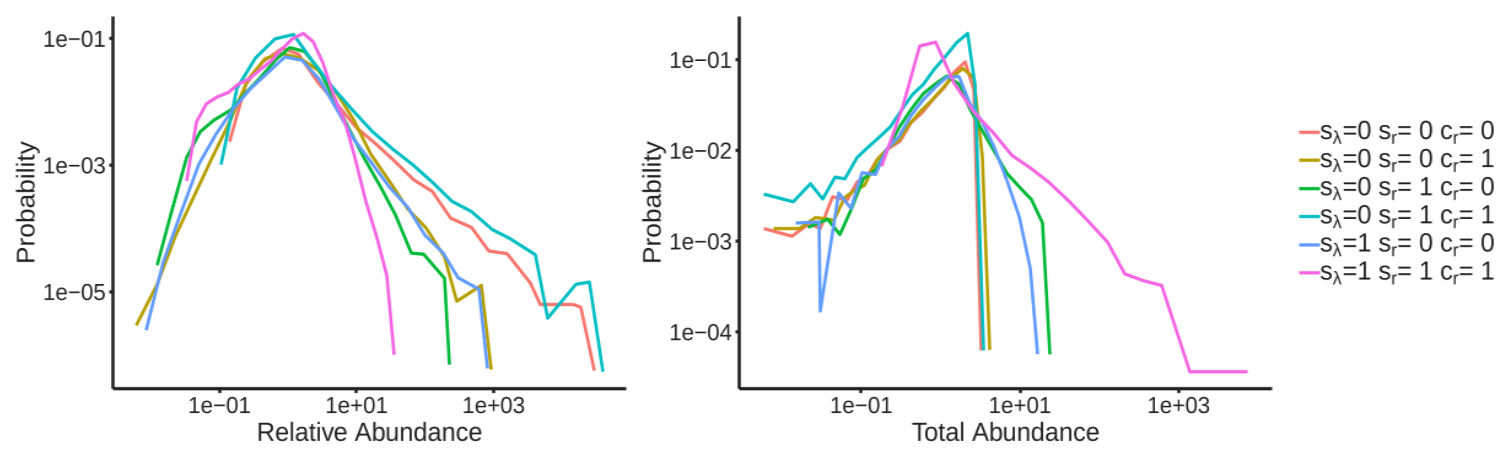

1522 Fig. S19. Numerical simulations of mathematical model \#4. The panels show the same

1523 distributions as in Fig. S16. The right plot shows the distribution of the total biomass across

1524 particles. In all the simulations we set $\lambda^{d}=1, \bar{\lambda}=1$, and $\bar{r}=1$ (where the total time of the

1525 experiment was also set to be equal to 1). Different values of $s_{r}, s_{\lambda}$ and $c_{r}$ correspond to different

1526 colors. 
1527 Table S1 (separate file). Metadata accompanying metagenome-assembled genomes (MAGs)

1528 from this study.

1529

1530 Table S2 (separate file). Metadata accompanying bacteriophage-annotated sequences from this

1531 study.

1532

1533 Table S3 (separate file). Metadata accompanying metabolomics performed in this study.

1535 Table S4 (separate file). Statistics on the distributions of bacteriophage-annotated sequences in 1536 accompanying metagenome-assembled genomes (MAGs) from this study according to the 1537 predicted MAG ecological role.

Table S5 (separate file). Metadata accompanying metagenomic samples collected in this study.

Table S6 (separate file). Accession numbers and methods for the creation of custom profile hidden

1542 Markov models (HMMs) used to annotate chitin metabolism-related genes in metagenome1543 assembled genomes in this study.

1545 Table S7 (separate file). Relative abundances of metagenome-assembled genomes (MAGs) in 1546 each sample collected in this study. metagenomics. Peerj 2016, 1-28 (2016). data. Bioinformatics 30, 2114-2120 (2014). 
4. S. I. Nikolenko, A. I. Korobeynikov, M. A. Alekseyev, BayesHammer: Bayesian clustering for error correction in single-cell sequencing. Bmc Genomics 14, S7 (2013). advanced methodologies and community practices. Methods 102, 3-11 (2016). recover genomes from multiple metagenomic datasets. Bioinformatics 32, 605-607 (2016). Methods 11, 1144-1146 (2014).

8. B. Langmead, S. L. Salzberg, Fast gapped-read alignment with Bowtie 2. Nat Methods 9, 357-359 (2012).

9. G. E. Leventhal, et al., Strain-level diversity drives alternative community types in millimetre-scale granular biofilms. Nat Microbiol 3, 1295-1303 (2018).

10. C. M. K. Sieber, et al., Recovery of genomes from metagenomes via a dereplication, aggregation and scoring strategy. Nat Microbiol 3, 836-843 (2018). Assessing the quality of microbial genomes recovered from isolates, single cells, and metagenomes. Genome Res 25, 1043-1055 (2015). substantially revises the tree of life. Nat Biotechnol 36, 996 (2018). genomes with the genome taxonomy database. Bioinformatics 36, 1925-1927 (2020).

14. W. J. Kent, BLAT---The BLAST-Like Alignment Tool. Genome Res 12, 656-664 (2002).

15. N. Otsu, A threshold selection method from gray-level histograms. leee Transactions Syst Man Cybern 9, 62-66 (1979).

16. R. C. Team, R: A Language and Environment for Statistical Computing (2021).

17. G. Pau, F. Fuchs, O. Sklyar, M. Boutros, W. Huber, EBImage-an R package for image processing with applications to cellular phenotypes. Bioinformatics 26, 979-981 (2010). 
18. D. Hyatt, et al., Prodigal: prokaryotic gene recognition and translation. (2020).

21. D. H. Haft, et al., TIGRFAMs: A protein family resource for the functional identification of proteins. Nucleic Acids Res 29, 41-43 (2001). 101, 2524-2529 (2004).

23. S. Eisenbeis, S. Lohmiller, M. Valdebenito, S. Leicht, V. Braun, NagA-dependent uptake of $\mathrm{N}$-acetyl-glucosamine and $\mathrm{N}$-acetyl-chitin oligosaccharides across the outer membrane of Caulobacter crescentus. J Bacteriol 190, 5230-5238 (2008). D515 (2019). Improvements in performance and usability. Mol Biol Evol 30, 772-780 (2013). Nucleic Acids Res 47, W636-W641 (2019). rapid micro-scale successions on model marine particles. Nat Commun 7, 1-7 (2016). Communities. Curr Biol 29, 1528-1535.e6 (2019).

29. S. Pontrelli, et al., Hierarchical control of microbial community assembly by specialists. 2079 (2009). Graphical Models (2019). 
1610

1611

1612

1613

1614

1615

1616

1617

1618

1619

1620

1621

1622

1623

1624

1625

1626

1627

1628

1629

1630

1631

1632

1633

1634

1635

32. B. Buchfink, C. Xie, D. H. Huson, Fast and sensitive protein alignment using DIAMOND. Nat Methods 12, 59-60 (2015).

33. S. Roux, F. Enault, B. L. Hurwitz, M. B. Sullivan, VirSorter: Mining viral signal from microbial genomic data. Peerj 2015, 1-20 (2015).

34. J. Ren, N. A. Ahlgren, Y. Y. Lu, J. A. Fuhrman, F. Sun, VirFinder: a novel k-mer based tool for identifying viral sequences from assembled metagenomic data. Microbiome 5, 69 (2017).

35. A. C. Gregory, et al., Marine DNA Viral Macro- and Microdiversity from Pole to Pole. Cell 177, 1109-1123.e14 (2019).

36. N. A. Ahlgren, J. Ren, Y. Y. Lu, J. A. Fuhrman, F. Sun, Alignment-free d2* oligonucleotide frequency dissimilarity measure improves prediction of hosts from metagenomicallyderived viral sequences. Nucleic Acids Res 45, 39-53 (2017).

37. R. A. Edwards, K. McNair, K. Faust, J. Raes, B. E. Dutilh, Computational approaches to predict bacteriophage-host relationships. Fems Microbiol Rev 40, 258-272 (2016).

38. A. R. Quinlan, I. M. Hall, BEDTools: A flexible suite of utilities for comparing genomic features. Bioinformatics 26, 841-842 (2010).

39. K. Kieft, K. Anantharaman, Deciphering active prophages from metagenomes. bioRxiv, 2021.01.29.428894 (2021).

40. M. R. Olm, et al., The source and evolutionary history of a microbial contaminant identified through soil metagenomic analysis. Mbio 8, 1-12 (2017).

41. N. Dumont-Leblond, M. Veillette, C. Racine, P. Joubert, C. Duchaine, Development of a robust protocol for the characterization of the pulmonary microbiota. Commun Biology 4 , $1-9(2021)$.

42. D. K. Button, B. R. Robertson, Determination of DNA Content of Aquatic Bacteria by Flow Cytometry. Appl Environ Microb 67, 1636-1645 (2001).

43. MATLAB, 9.7.0.1190202 (R2019b) (The MathWorks Inc.). 
bioRxiv preprint doi: https://doi.org/10.1101/2021.09.27.461956; this version posted September 27, 2021. The copyright holder for this preprint (which was not certified by peer review) is the author/funder, who has granted bioRxiv a license to display the preprint in perpetuity. It is made available under aCC-BY-NC-ND 4.0 International license.

1636

1637

1638

1639

1640
44. T. Fuhrer, D. Heer, B. Begemann, N. Zamboni, High-throughput, accurate mass metabolome profiling of cellular extracts by flow injection-time-of-flight mass spectrometry. Anal Chem 83, 7074-7080 (2011).

45. P. D. Karp, et al., The BioCyc collection of microbial genomes and metabolic pathways. Brief Bioinform 20, 1085-1093 (2017). 